\title{
The effect of the decorator crab Notomithrax minor on cultivated Greenshell mussel spat (Perna canaliculus) survival, growth and byssal characteristics.
}

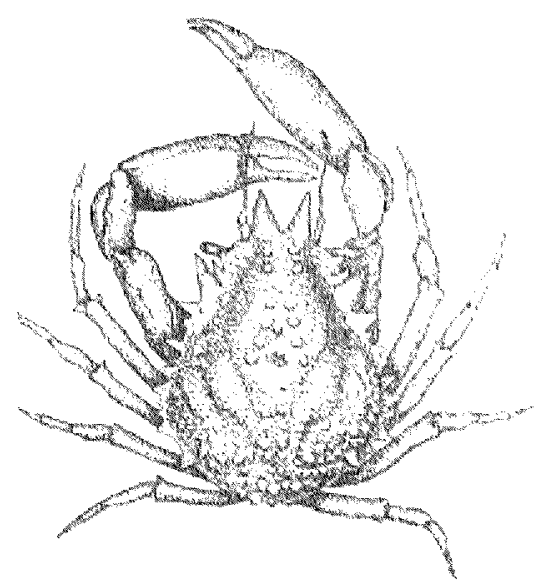

By

Irene van de Ven

\begin{abstract}
A thesis
submitted to the Victoria University of Wellington in partial fulfilment of the requirements for the degree of Master of Science in Marine Biology
\end{abstract}




\section{Acknowledgements}

I would like to thank my supervisors Jonathan Gardner and Kevin Heasman for their support, mentoring advice and patience.

I would also like to acknowledge the financial support received through the Dick and Mary Earle Scholarship in Technology, it proved one of the vital aspects in being able to produce this research.

Thank you to Jorge David Aguirre Davies and Chris Cornwall for the many hours spent diving in dark, inhospitable and just plain nasty conditions underneath wharfs in the Wellington harbour to collect crabs.

Thanks to the employees of Sanford Ltd; in particular Dave Herbert for making spat available for my experiments and for organising transport to and from my study sites. I would also like to thank Aaron Pannell and Dean Higgins at Marlborough Mussel Company for initially showing interest in my topic and for the numerous trips on their mussel harvesters and other boats. They also made a huge amount of spat available for experiments and made it possible for me to present my preliminary findings at the New Zealand Marine Farming Association AGM. Without this cooperation from the Greenshell mussel industry this project could never have been accomplished. 
Thanks to the staff at the Cawthron Institute in particular the Cawthron Aquaculture Park staff; Dan McCall, Ellie Watts, Norman Ragg, Henry Kaspar and Achim Janke for providing invaluable advice, logistic and moral support and resources.

I would also like to thank Steve Pether at NIWA Bream Bay for being so understanding and giving me so many days off during the final writing up period of my thesis.

Thanks to Colleen Kelly, Nokuthaba Sibanda and Jorge David Aguirre Davies for statistical advice.

Last but definitely not least my family for your support and encouragement throughout. Thank you for putting up with the seemingly endless late night phone calls and stressful days and nights, I would never have made it to where I am today without the love and understanding of my mum, dad, sister and my partner Nick. Special thanks to Nick for helping me count thousands of mussels, measure hundreds of crabs and scrub dozens of mussel-lines. 


\begin{abstract}
The decorator crab Notomithrax minor is common on Greenshell mussel (Perna canaliculus) farms in the Marlborough Sounds, New Zealand. Individuals in the Greenshell mussel industry have suggested that the presence of $N$. minor, found on mussel lines, is related to substantial losses of Greenshell mussel spat.

Laboratory and field investigations were used to assess the effect of $N$. minor presence on the retention and productivity of Greenshell mussel ${ }^{\mathrm{TM}}$ spat. Specific consideration was given to predation pressure and induced anti-predator defenses, both of which can cause financial losses to mussel farmers. High (12 crabs/cage $\left.{ }^{-1}\right)$ and low (3 crabs/cage ${ }^{-1}$ ) densities of large (males: $>20 \mathrm{~mm}$, females: $>15 \mathrm{~mm}$ TCW) and medium (males: $15-20 \mathrm{~mm}$, females: $10-15 \mathrm{~mm}$ TCW) decorator crabs were placed in cages on commercial Greenshell mussel farm droppers at two sites in the Pelorus Sound. The byssal characteristics, spat retention rate and spat shell length were assessed at 8 and 11 weeks after trial initiation.
\end{abstract}

Greenshell mussel density on the experimental droppers decreased significantly when medium and high densities of the decorator crabs ( $N$. minor) were introduced. $N$. minor presence induced the remaining Greenshell mussel spat to produce more and thicker byssus threads which consequently lead to increased mussel attachment. The decrease in retention rate and the increase in mussel attachment strength were more pronounced in small recently seeded spat.

Laboratory experiments to assess the consumption rate of small $(\leq 5 \mathrm{~mm})$ Greenshell mussel spat by decorator crabs showed that mussel consumption by $N$. minor peaked at $56.43( \pm 13.02(95 \%$ C.I. $)) \mathrm{crab}^{-1} \mathrm{hr}^{-1}$, however the rate of consumption 
decreased significantly over the duration of the three day trial. $N$. minor prey size preference was also assessed using Laboratory trials; crabs were offered 4 size classes of mussels (small $(<5 \mathrm{~mm})$, small-medium $(5-10 \mathrm{~mm})$, medium-large $(10-15 \mathrm{~mm})$, large $(>15 \mathrm{~mm})$ simultaneously. Female crabs consumed more mussels in the $<5 \mathrm{~mm}$ and $5-10$ $\mathrm{mm}$ size classes than in the two larger mussel size classes $(10-15 \mathrm{~mm}$ and $>15 \mathrm{~mm})$, whereas the male crabs showed a numerical preference for mussel spat in the smallmedium and medium-large size classes.

This study provides preliminary evidence that the decorator crab $N$. minor is a previously overlooked and under-estimated threat to the Greenshell Mussel industry in the Marlborough Sounds that deserves closer scrutiny and experimental testing. 


\section{Table of Contents}

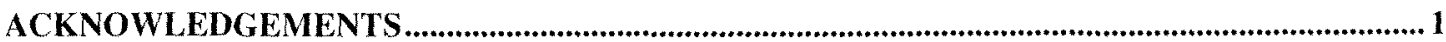

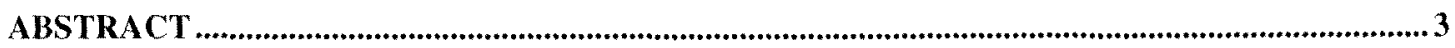

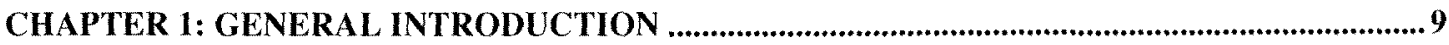

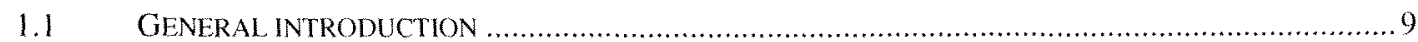

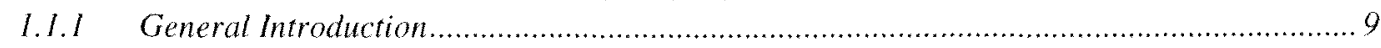

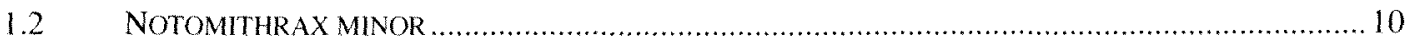

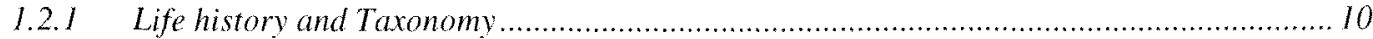

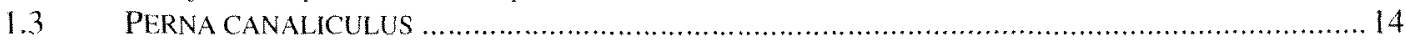

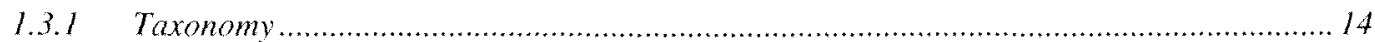

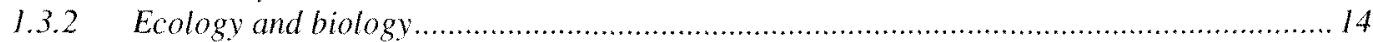

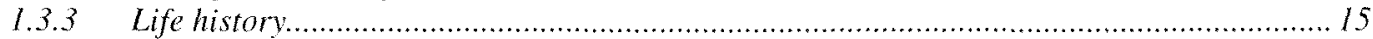

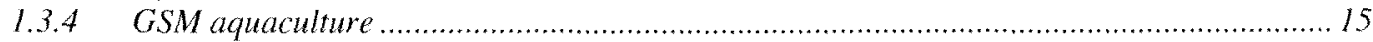

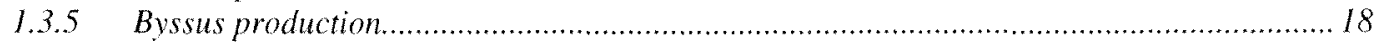

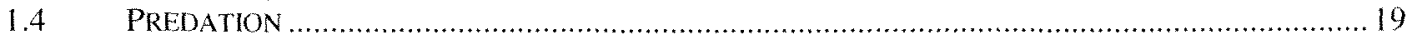

1.4.1 Crab predation and inducible anti-predator defenses................................................ 19

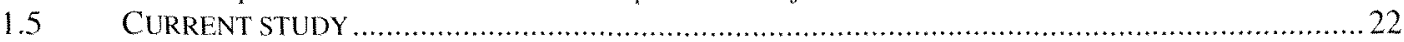

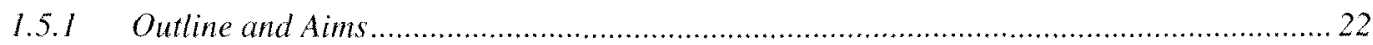

CHAPTER 2: PREDATOR INDUCED CHANGES IN GREENSHELL MUSSEL BYSSAL

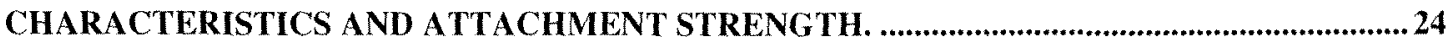

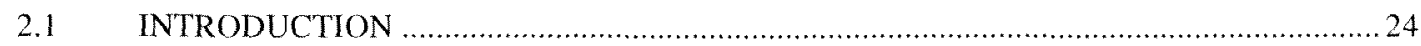

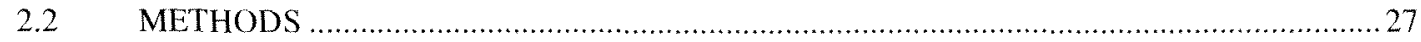

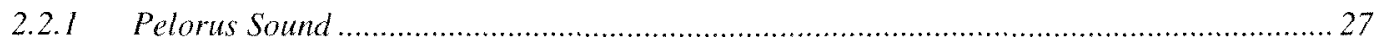

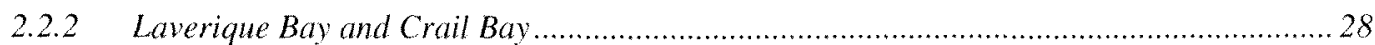

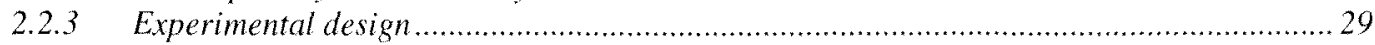

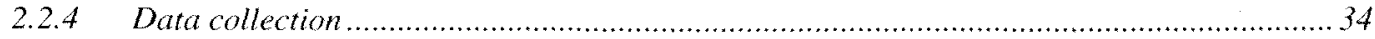

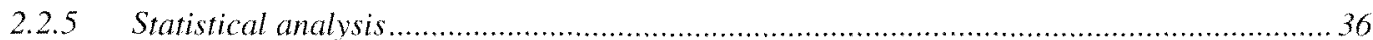

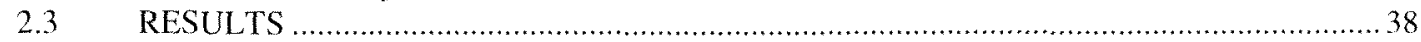

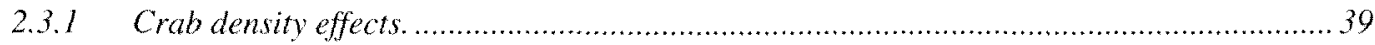

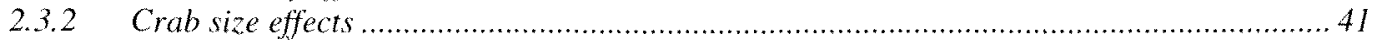

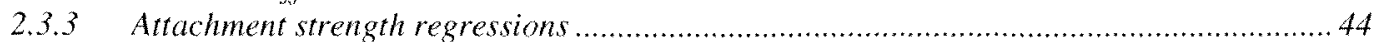

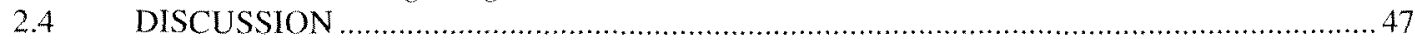

CHAPTER 3: GREENSHELL MUSSEL PREDATION BY NOTOMITHRAX MINOR: SPAT RETENTION, PREY SIZE SELECTION AND PREDATION RATES ..............................................51

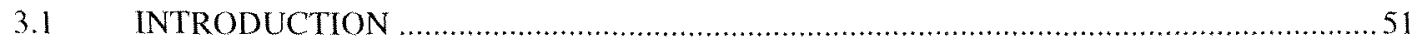

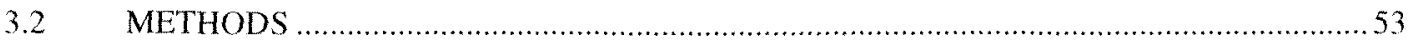

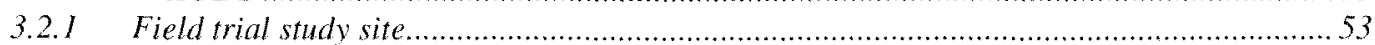

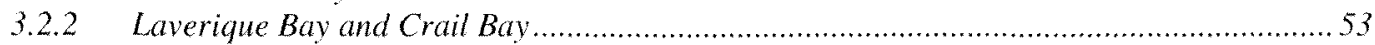

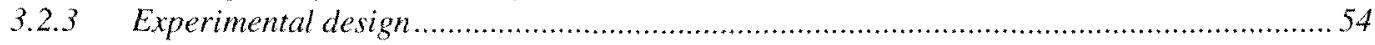

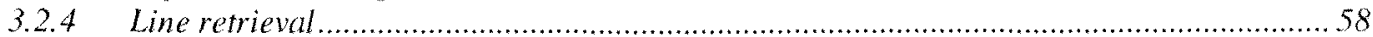

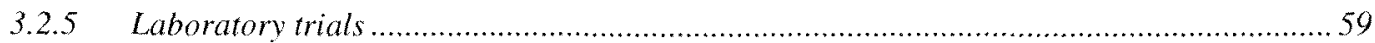

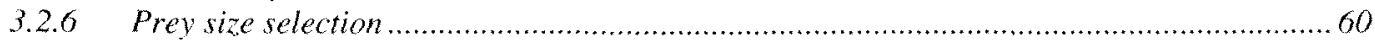

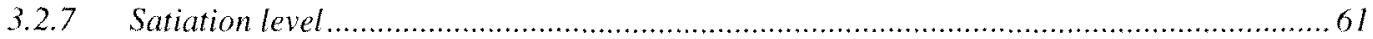

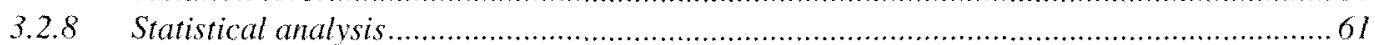

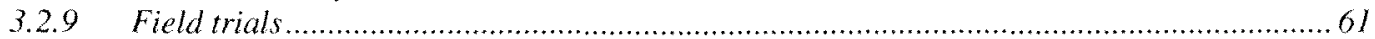

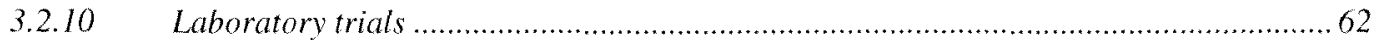

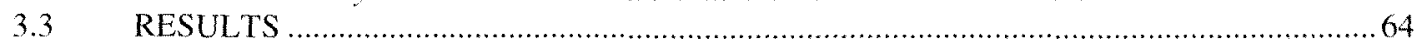

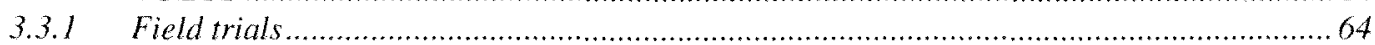

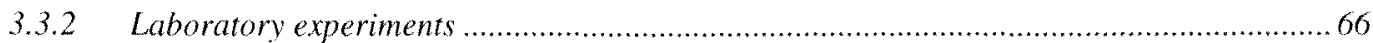




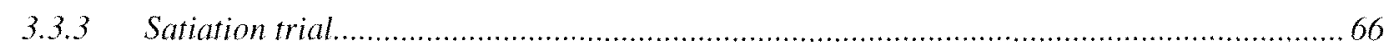

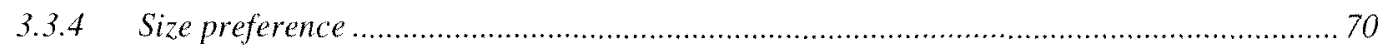

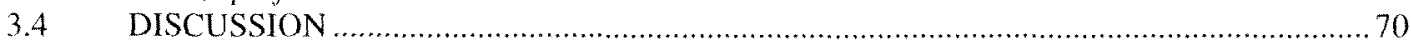

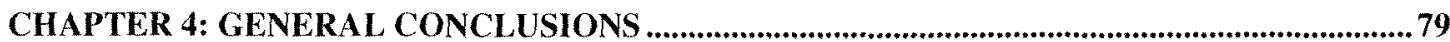

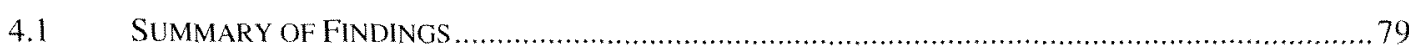

4.1.1 N. minor and GSM spat byssus production ................................................................. 79

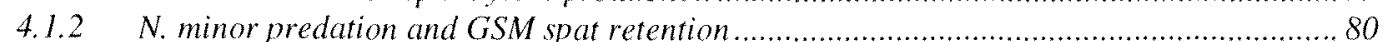

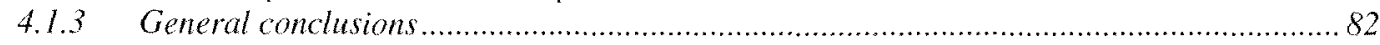

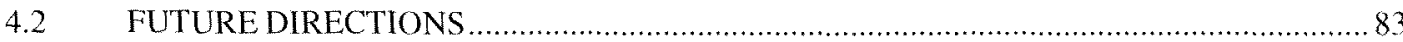




\section{Table of Figures}

Figure 1.1: The three species of Notomithrax found in New Zealand waters; 1) Notomithrax minor the smallest of the three (TCW $>32 \mathrm{~mm}$ ) and most common Notomithrax spp. on mussel lines in the Marlborough Sounds, 2) Notomithrax peronii is the largest species (TCW> $65 \mathrm{~mm}$ ) and is occasionally found on old mussel lines and 3) Notomithrax ursus commonly known as the hairy seaweed crab is relatively rare on mussel lines in the Marlborough Sounds. $N$. minor and $N$. peronii are often confused for large or small individuals of the other species. Figure adapted from Griffin 1966.

Figure 1.2: The Marlborough Sounds, the primary area for aquaculture of the New Zealand Greenshell mussel. Also shown is Ninety Mile Beach, the most prominent GSM spat harvest area in New Zealand.

Figure 2.1: Mean readings of salinity, water temperature, Chl $a$ and suspensoids for an inner and an outer Sound (relative to the ocean) site in the Pelorus Sound, New Zealand. Modified from (Hickman et al. 1991a).......

Figure 2.2: schematic illustration of the construction of a GSM mussel farm in the Marlborough Sounds, outlining terms in the methodology (study site, backbone, dropper and cage) and the positioning of the experimental cages.

Figure 2.3: The location of the study sites. Boxes indicate site locations within Pelorus Sound, Crail Bay to the south (2) and Laverique Bay to the north (1). Locations of existing mussel farms are from Marlborough District Council data (2003)....

Figure 2.4: An example of a Greenshell ${ }^{\mathrm{TM}}$ mussel byssus thread magnified 40x, indicating the location of measurements recorded for the analyses. 1: 'stem' diameter, 2: 'byssus' diameter.

Figure 2.5: Effects of potential predator ( $N$. minor) density on Greenshell mussel byssal stem thickness (mean \pm 95\% C.I.) and byssus number at the two study sites, Crail Bay and Laverique Bay. Stem thickness and Byssus number were standardized to days by dividing the total number by the number of days the experiment ran for ( 42 and 77 days at Laverique Bay, 56 days for Crail Bay). At Crail Bay the caged and un-caged controls had significantly thinner byssal stems and lower byssus numbers than the average and high crab density treatments. The differences in stem thickness and byssus number were not statistically significant at Laverique Bay (ANOVA). Letters represent statistically significant post hoc groupings calculated using Tukey tests and ANOVA analysis, only Tukey test results for significant ANOVA results are displayed. Crab density treatments are as follows: UC- un-caged control, CC-caged control, Average- 3 crabs per cage, High- 12 crabs per cage. . . . . . . . . . . . . . . . . . . . . . . . 40

Figure 2.6: Effects of potential predator (N.minor) size on Greenshell ${ }^{1 m}$ mussel stem thickness, byssus number and attachment strength (mean $\pm 95 \%$ C.I.) at the two study sites, Crail Bay and Laverique Bay. Stem thickness, byssus number and attachment strength were standardized to days by dividing the total number by the number of days the experiment ran for (42 and 77 days at Laverique Bay, 56 days for Crail Bay). At Crail Bay the caged and un-caged controls had significantly thinner byssal stems and less byssus threads than the average and high crab density treatments. At both study sites mussels in the crab treatments (medium and large) had significantly higher attachment strengths than control mussels (CC and UC). Letters represent post hoc groupings calculated using Tukey tests, only Tukey test results for significant ANOVA results are displayed. Crab size treatments are as follows: UC-un-caged control, CC-caged control, Medium and Large crabs.

Figure 2.7: Slopes of regression of GreenshellTM mussel attachment strength against the three byssus characteristics included in the multiple linear regression analysis ( $\pm 1 \mathrm{SE}$ ) for each study site.

Figure 2.8: the effects of Greenshell ${ }^{\mathrm{m}}$ mussel byssus number on the attachment strength of individual mussels at two study sites. Attachment strength is measured in grams of pressure required to dislodge mussels from the mussel spat ropes. $R^{2}$ values and equations shown in figure. 
Figure 3.1: The location of the study sites. Boxes indicate site locations within Pelorus Sound, Laverique Bay to the north (1) and Crail Bay to the south (2). Locations of existing mussel farms are from Marlborough District Council data (2003)

Figure 3.2: schematic illustration of the construction of a GSM mussel farm in the Marlborough Sounds, outlining terms in the methodology (study site, backbone, dropper and cage) and the positioning of the experimental cages.

Fig 3.3: Effects of potential predator (N. minor) presence on P. canaliculus (Greenshell mussel) density (mussels $\mathrm{m}^{-2}$ ) on spat catching ropes at the two study sites, Crail Bay and Laverique Bay. Mussel number was standardised for days by dividing the mussel number by the number of days the experiment ran for ( 42 and 77 days at Laverique Bay, 56 days for Crail Bay). The differences in P. canaliculus densities were statistically significant at Crail Bay but not at Laverique Bay. Letters represent statistically significant post hoc groupings calculated using Tukey tests following ANOVA. Crab density treatments are as follows: UC - un-caged control, CC - caged control, Average - 3 crabs per cage, High -12 crabs per cage.

Fig 3.4: Effects of crab (N. minor) presence on P. canaliculus (GSM) size on spat catching ropes at the two study sites, Crail Bay and Laverique Bay. Mussel size was standardised for days by dividing the mussel number by the number of days the experiment ran for (42 and 77 days at Laverique Bay, 56 days for Crail Bay). The differences in P. canaliculus size were statistically significant at Crail Bay but not at Laverique Bay (ANOVA). Letters represent statistically significant post hoc groupings calculated using Tukey tests following ANOVA

Fig 3.5: Average percent of GSM spat consumed by medium-sized male and female $N$. minor (males 20-35 mm TCW; females 15-27 mm TCW) and the number of mussels lost in control treatments (no crabs) over three days. Means $\pm 95 \% \mathrm{Cl}$

Fig 3.6: Average number of Greenshell ${ }^{\mathrm{m}}$ mussel $(P$. canaliculus $)$ spat $(<5 \mathrm{~mm})$ eaten per medium sized male and female crab ( $N$. minor minor: males: $20-35 \mathrm{~mm}$ TCW , females: $15-27 \mathrm{~mm}$ TCW) per hour across three days of testing. Error bars are $95 \%$ confidence intervals of the mean

Fig 3.7: Average number of GSM (P. canaliculus) spat $(<5 \mathrm{~mm})$ eaten by male and female medium sized crabs $(N$. minor: males: $20-35 \mathrm{~mm}$ TCW, females: $15-27 \mathrm{~mm}$ TCW) per day across three days of testing. Means $\pm 95 \% \mathrm{Cl} . \ldots . . .69$ 


\section{Chapter 1: General Introduction}

\subsection{GENERAL INTRODUCTION}

Predation can have significant effects on the structure, distribution and abundance of prey populations (Krebs, 1985). For example, Menge (1979) found that invertebrate predators contribute to the production of free space in the rocky inter-tidal zone in New England by limiting the abundance of the dominant space occupier, the blue mussel, Mytilus edulis. The availability of free space is a fundamental determinant of species diversity in this system; consequently the predator indirectly influences community structure.

Because crabs are common predators in many marine ecosystems their behavior and feeding activities often seriously affect not only prey species, but also non-target coexisting organisms (Woods 1993). Direct and in-direct effects of predator behavior (which can include the stimulation of defense or escape strategies among the prey species) may disrupt the fitness of prey individuals and subsequently the persistence of prey species (Leonard et al. 1999, Mistri 2004). Brachyuran crabs are voracious predators and prey heavily on marine bivalves, including many mussel species (Mistri 2004). Due to their prolific nature and cosmopolitan distribution, it is important to understand crab prey preferences and how the predator influences the behavior and morphology of prey species, particularly when the crabs or their prey are commercially exploited (Woods 1993).

In this study the focal prey species, the endemic New Zealand GSM Perna canaliculus, is one of the most extensively farmed marine species in New Zealand 
waters, and the focal predator species, the camouflage crab Notomithrax minor, has no direct commercial value. However, this predator may have important indirect commercial values due to the possible loss of mussels resulting from direct predation and/or indirect dislodgement from aquaculture farms. Therefore this study aims to assess the impact $N$. minor presence has on the growth, attachment rate and survival of farmed GSMs in the Marlborough Sounds and the resulting economic and ecological implications.

\subsection{NOTOMITHRAX MINOR: LIFE HISTORY AND TAXONOMY}

Globally the majidae family (spider crabs) is represented by at least 19 species from 12 genera and 4 subfamilies. The subfamily Majinae includes the genus Notomithrax, of which three species occur in New Zealand waters, Notomithrax minor, Notomithrax peronii and Notomithrax ursus. N. minor is the smallest species with females reaching a maximum carapace width of $24 \mathrm{~mm}$ and males $31.5 \mathrm{~mm}$. The species was first described as Paramithrax minor (Filhol 1885), but this taxonomic classification has been revised many times before the most recent designation as N. minor in 1966 (Griffin).

In New Zealand N. minor is distributed from Cape Maria van Diemen to Stewart Island, and throughout southeast Australia including Tasmania (Griffin 1966). It has a bathymetric range generally extending from the inter-tidal to $40 \mathrm{~m}$ and only rarely to 90 m (Wear and Webber 1981).

$N$ minor is cryptic by nature and like many other spider crabs its movement is relatively sluggish: these crabs generally rely on masking ability for camouflage, rather than on speed for predator avoidance. The carapace of $N$. minor is commonly adorned 
with sponges and branching algae, but crabs will mask with other materials including mussel rope filaments and the moults of con-specifics (pers. obs.). Using their chelae, the crabs generally tear pieces of sponge or algae to a certain size. One side of the selected item is then manipulated with the mouthparts before it is brushed against the hooked setae on the carapace so that the item adheres (Wicksten 1980, Sato and Wada 2000). Due to this masking ability and the cryptic nature of the crab, the species distribution and density is frequently underestimated (Bennett 1964).

N. minor is commonly found in association with the larger Notomithrax peronii on sand, mud and coarse shell reefs in sheltered areas such as bays and harbours (Wear and Webber 1981). Both N. minor and N. peronii are also found in high densities on and around artificial substrates such as wharf pilings and mussel aquaculture structures (pers. obs.).

Reproduction is aseasonal with ovigerous females observed from September to April, although at Leigh (north of Auckland, and now the site of the Cape Rodney to Okakari Point Marine Reserve) ovigerous females have been found in June and July (Griffin 1966). Newly spawned eggs are bright orange, but when ready to hatch the eggs fade to light orange or are transparent (Griffin 1966). Once eggs hatch they go through 4 larval stages: a pre-zoeal stage, 2 zoeal stages and a megalopa larva. Each of the zoeal stages lasts approximately 9 days and all stages are most abundant during spring and summer (Wear and Webber 1981). 


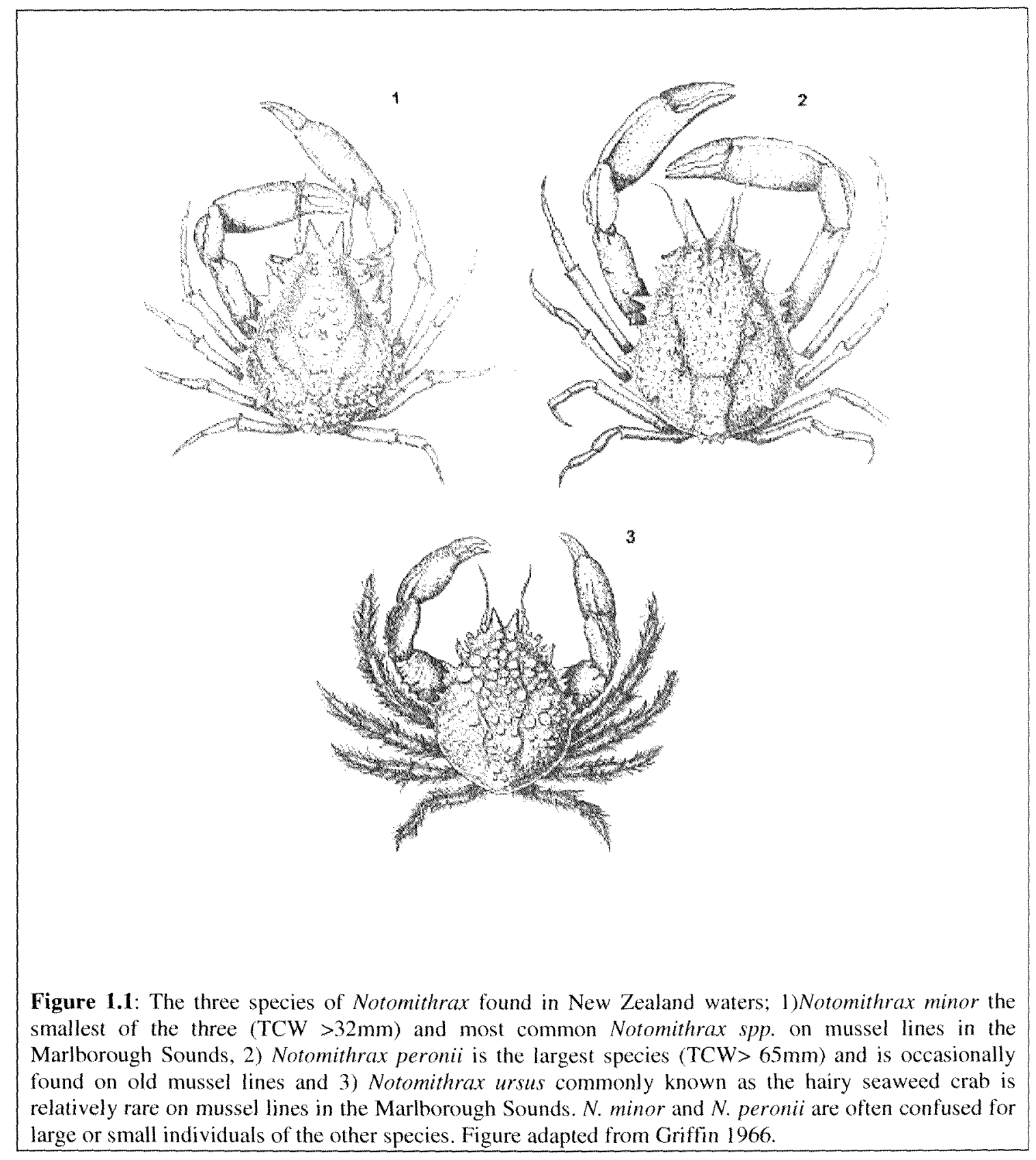

The majority of published data regarding $N$. minor and other spider crab species describes the crabs masking ability and use of the camouflaging items as a food source (Wicksten 1980, Kilar and Lou 1986, Woods and McLay 1994, Sato and Wada 2000, Cruz-Rivera 2001). Very little published data are available regarding the diet, behavior and growth of $N$. minor, although other species of Notomithrax have been described as 
omnivorous. Woods (1993) researched the natural diet of $N$. ursus and found that it consisted of a wide range of items including: algae, gastropods, isopods, amphipods, bryozoans, sponges, bivalves and small decapods. Omnivorous feeding patterns have been observed in other species of spider crabs, with algae often constituting a large part of the diet. For example, the spider crab Loxorhynchus crispatus is an omnivore that is found off the central Californian coast. Hines (1982) established that it feeds in a variety of items including: algae, sipunculids, sponges, polychaetes, gastropods and other crustaceans. Current evidence suggests that bivalves only constitute a small portion of the diet of spider crabs. This may be due to the morphology of the chelae which are generally elongate and slender (Wicksten 1980, Woods 1993) and are not well adapted to the crushing behaviour required to break the bivalves relatively thick shells (Woods 1993). When N. ursus and $N$. minor do prey on bivalves they generally use one of two techniques: 1) chipping or biting the shell margin using chelae or mandibles, or 2) forcing the valves of the bivalve apart using the chelae or legs (Woods 1993); pers. obs.).

The settlement of both $N$. minor and $P$. canaliculus is highly irregular however N. minor megalopa larvae settle slightly later in the year (October to March (Griffin 1966)) than GSM spat (August to February (Jeffs et al. 1999)). Therefore N. minor may settle onto the mussel lines soon after the mussel spat has been seeded and grow along side the mussel spat. When the spat gets stripped from the spat lines for reseeding (refer to 1.3.4) the crabs also get removed and either a second wave of settlement may occur, or older crabs may migrate from adjacent lines or floats. There is little data regarding how the settlement patterns of $N$. minor onto the GSM farms and their adaptations to this 
relatively instable environment. To make any concrete assumptions regarding these issues will require further investigation.

\subsection{PERNA CANALiCULUS}

\subsubsection{Taxonomy}

The GSM, Perna canaliculus, is endemic to New Zealand and is one of at least nine different species of Mytilid found in New Zealand waters (Powell 1979). $P$. canaliculus has often been referred to as the green-lipped mussel because of its characteristic green shell margin (Jeffs et al. 1999). It is now more commonly known by its trade-name, the GSM.

The species was first described as Mytilus canaliculus by Martyn in 1784, but not recognized until 1791 (Gmelin) (Siddall 1980, Jeffs et al. 1999). It was proposed that the species be placed in the genus Perna (Retzius 1788) in 1959. Other members of the genus Perna are found in South America, Africa (Perna perna Linneaus 1758) and the Indo Pacific (Perna viridis Linneaus 1758) (Jeffs et al. 1999). For the purpose of this study I will refer to P. canaliculus as GSM (GreenshellTM mussel).

\subsubsection{Ecology and biology}

The GSM is widely distributed throughout New Zealand from $\sim 35^{\circ} \mathrm{S}$ to $\sim 47^{\circ} \mathrm{S}$ (Jeffs et al. 1999, Apte et al. 2003), and is typically found from the midlittoral to depths of over $50 \mathrm{~m}$ (Powell 1979). The GSM can often form dense beds of up to $100 \mathrm{~m}^{-2}$ (Jeffs et al. 1999) that filter significant quantities of suspended organic matter and plankton out of the water. The distribution of the GSM is restricted in the inter-tidal zone at the upper limit by desiccation stress and in the shallow sub-tidal region at the lower limit by predation pressure (Paine 1969). Although GSMs are susceptible to desiccation stress it 
does tolerate a wide range of water salinities, from 25 ppt to $35 \mathrm{ppt}$ (Flaws 1975, Jeffs et al. 1999), and temperatures, from $5.3^{\circ} \mathrm{C}$ to $27^{\circ} \mathrm{C}$ (Hickman et al. 1991b, Jeffs et al. 1999)

\subsubsection{Life history}

Because of its ecological, cultural and economic importance, the life history of the GSM has been studied intensively. The following is a brief overview. GSMs are dioecious broadcast spawners. Gonadal development and spawning are temperature and food dependant, with most spawning occurring from late spring to early autumn (Jeffs et al. 1999). Females can produce up to 100 million eggs, and once fertilized the eggs develop into lecitotrophic trochophore larvae, followed by early veliger development at 24-48 hr (Hayden 1994). The planktotrophic larval veliger stage feeds on a range of microalgae for 3-5 wk, during which time the prodissoconch II forms and grows to 100 $250 \mu \mathrm{m}$. Pediveligers $(220-350 \mu \mathrm{m})$ develop at $4-6 \mathrm{wk}$ and settle preferentially on fine filamentous substrata, including hydroids and filamentous algae (Manning 1985a,b, Buchanan 1994a). Primary settlement of the larvae is complete at byssogenesis, at which stage the mussels recruit into beds of adult mussels during secondary settlement through byssopelagic movement, or mucus drifting (Buchanan 1994). The ability for byssopelagic movement is lost once the spat reach $6 \mathrm{~mm}$ in length (Jeffs et al. 1999). Post settlement growth rates vary due to differences in phytoplankton supply and water temperature, but averages around 110-115 mm pa (Jeffs et al 1999).

\subsubsection{GSM aquaculture}

GSMs have been cultured in New Zealand since the late 1960s, with the Marlborough Sounds being the center of the industry (Dawber 2004). The Marlborough Sounds are located in the northeast of the South Island (from $40^{\circ} 53^{\prime} \mathrm{S}$ to $41^{\circ} 18^{\prime} \mathrm{S}$ and 
from $173^{\circ} 45^{\prime} E$ to $174^{\circ} 23^{\prime} E$ ) and consist of two major Sounds, the Pelorus and the Queen Charlotte (Fig. 1.2) (Jenkins 1979).

The GSM industry in New Zealand evolved through a succession of steps inspired by mussel farming patterns and practices in Spain, Japan and America (Dawber 2004). Initial attempts at culture were based on Spanish raft techniques and were generally unsuccessful due to unwieldy, hazardous and unstable rafts (Jenkins 1979, Jeffs et al. 1999, Dawber 2004).

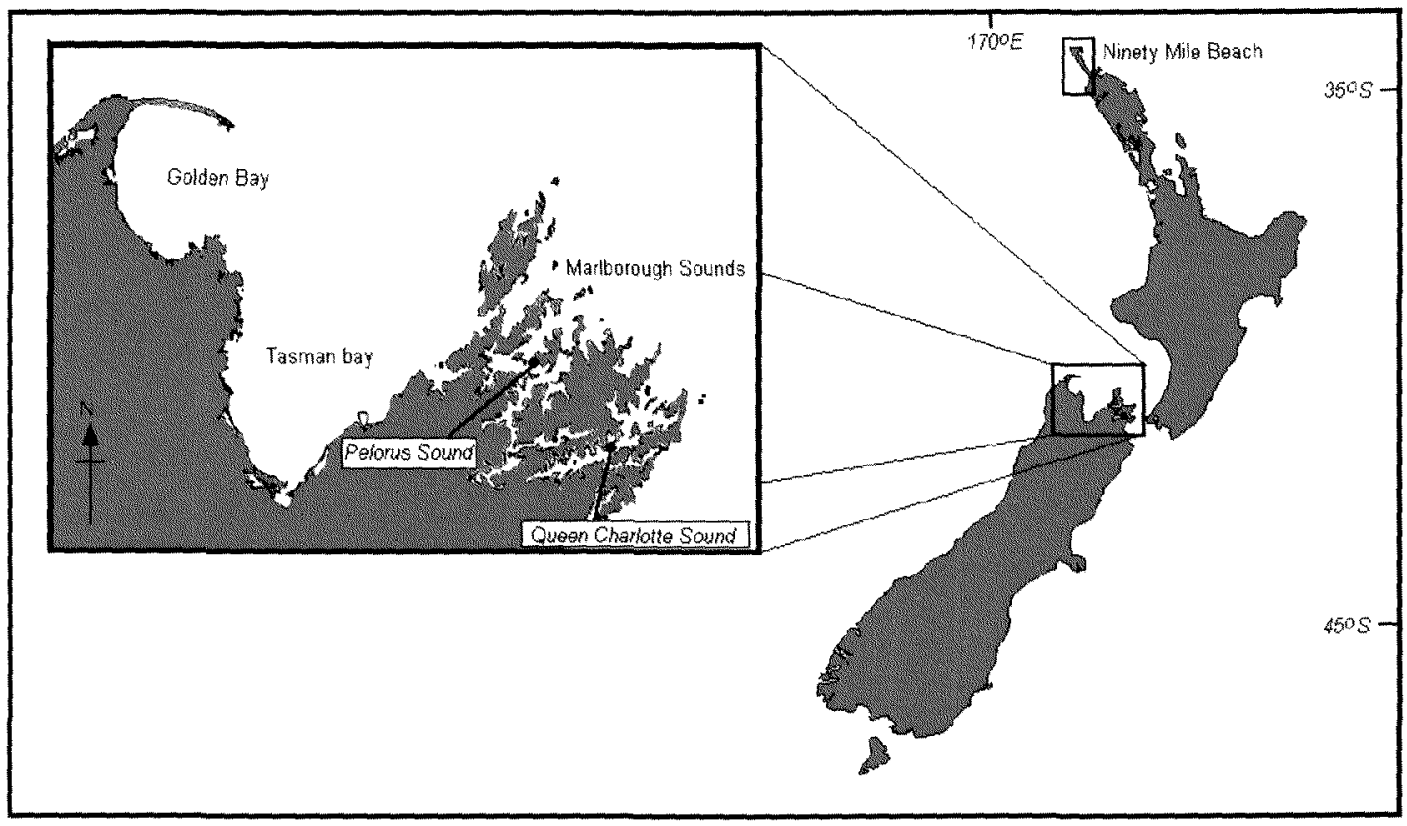

Figure 1.2. The Marlborough Sounds, the primary area for aquaculture of the New Zealand Greenshell mussel. Also pictured is Ninety Mile Beach, the most prominent GSM spat harvest area in New Zealand.

Following failed trials of raft culture, development of the Japanese long-line cultivation system started to take place. This system made more efficient use of water space, was flexible, and had the option of adding more buoyancy as the mussels grew heavier (Dawber 2004). Consequently the first considerable mussel harvest of 300 tonnes was produced in 1977 using a modified version of the Japanese long-line system. 
Expansion, refinement and increased mechanization have led to constant increases in production of mussels and the value of the industry. Currently, GSM production accounts for $\sim 5 \%$ of global mussel production (Carton et al. 2007) with an export market revenue of more than NZ\$ $188 \mathrm{M}$. GSM production is now New Zealand's most valuable seafood export, having surpassed other exported species such as hoki, orange roughy and rock lobsters (SeaFIC 2007).

The main problem faced by the industry is the very considerable reliance on wild caught spat (seed mussels). Approximately $80 \%$ of the spat comes ashore attached to macro-algae that are washed up on Ninety Mile Beach (Northland, New Zealand (Fig.1.1)). Following collection of the seed mussels from Ninety Mile Beach they are transferred and held on cultivation ropes in the Marlborough Sounds with a biodegradable stocking. The New Zealand GSM industry is acutely aware that it is in large part reliant on an unpredictable source of wild-collected spat, a supply which may or may not continue into the future. The remaining $20 \%$ of spat are caught on fibrous ropes suspended near existing mussel farms in the Marlborough Sounds and Golden Bay (Fig. 1.1) (Jeffs et al. 1999, Carton et al. 2007). Spat catching can be difficult due to the variable nature of larval settlement and because there is also high predation, mortality and secondary settlement among newly settled mussels.

Competition for space and nutrients by fouling organisms such as the blue mussel Mytilus galloprovincialis, and algae such as Ulva spp., Undaria pinnatifida and various ascidians also leads to losses in spat number and quality (Hickman 1979, Hayden 1984). Environmental factors including significant variation in water temperature and salinity may also cause variations in the viability and retention of spat (Sim-Smith 2006). These and other issues contribute to only about $2 \%$ of all mussel spat 
applied to grow-out ropes remain and grow to a harvestable size (Webb and Heasman 2006).

GSM spat costs mussel farmers NZ\$0.80 (Kaitaia spat) and NZ\$1.00 (locally caught and Golden Bay spat) per $\mathrm{m}$ of dropper (at a density of 200 per $\mathrm{m}$ ) (personal communication, Aaron Pannell, Marlborough Mussels Ltd., 2007) so poor spat retention costs the aquaculture hundreds of thousands of dollars annually (Sim-Smith 2006). The mussel farmers must purchase and seed excessive amounts of spat to compensate for spat losses, the cost to the farmers also increases when hatchery spat is used to compensate for short comings in wild spat catches. The issue of spat retention is incredibly complex, and to make solving this issue possible it is important for the industry to identify and quantify the factors leading to spat losses. Predation has been identified by NIWA and Sealord Group Ltd. as one of the factors leading to decreases in spat retention. Therefore the current study into the effects of $N$. minor on Greenshell ${ }^{\mathrm{TM}}$ spat could potentially be very valuable to the mussel aquaculture sector.

\subsubsection{Byssus production}

Mussels attach to the substratum by byssus threads which are extracellular, collagenous structures secreted by the mussel foot (Carrington 2002). A byssus consists of three different parts: the 'root', which is fixed to the base of the foot and connects the rest of the byssus to the byssus retractor muscles; the 'stem', which is an extension of the root outward and supports the separate byssal threads; and the 'byssal threads' which radiate from the stem and attach to the substrate via adhesive disks or plaques (Carrington 2002). 
Byssus threads are not produced continuously but their production responds, with a lag time, to numerous environmental stimuli (Côte 1995). For example, there is evidence that factors such as wave action (Young 1985, Dolmer 1998, Carrington 2002), salinity (Young 1985), temperature (Christophersen and Strand 2003, Carton et al. 2007), and oxygen concentration (Alfaro 2005) may all cause an increase in the number of byssus threads produced, and that this thread number is the primary determinant of attachment strength (Bell and Gosline 1996). Increased byssus thread production has been attributed to anti-predator defense strategies particularly in relation to predatory decapods. By increasing the strength of byssal attachment the chance of being dislodged and consumed a crab is reduced.

\subsection{PREDATION}

\subsubsection{Crab predation and inducible anti-predator defences}

Anecdotal evidence from individuals in the mussel industry in the Marlborough Sounds and at The Cawthron Institute (Nelson) suggests that the presence of $N$. minor found on mussel lines is related to substantial losses of GSM spat. The crabs may also affect the amount of fouling on the mussel ropes and mussel attachment strength.

Because of the commercial nature of aquaculture practices, the effects of a predator are only investigated if they are known or are thought to have the potential to impact on the viability and economic value of the farmed product. Aquaculture practices usually create a monoculture of the farmed species. As a consequence, predation can cause direct losses due to mortality or by damaging large quantities of the farmed 'crop'. Predation can also indirectly lead to losses by causing the farmed animal to convert energy primarily allocated to growth into development of behavioral and physiological anti-predator defenses. Many of the bivalves that fall prey to crabs are relatively sessile 
and hence lack the ability to flee the predator. Alternative methods of predator avoidance have evolved in many of these species, including increased shell thickness (Reimer and Tedengren 1996, Reimer and Harms-Ringdahl 2001, Cheung et al. 2004), variations in shell allometry (Reimer and Tedengren 1996), shell allometry (Cheung et al. 2004) and increased strength of attachment (Côte 1995, Leonard et al. 1999, Reimer and Harms-Ringdahl 2001, Cheung 2004), all of which increase the handling time by crabs and which in turn decreases the 'profitability' of the prey item (Côte 1995). Under the energy maximization premise, the dietary value of a prey item is measured by the ratio of $\mathrm{E} i / \mathrm{l} i$, where $\mathrm{E} i$ is the energy units prey item $i$ yields and $\mathrm{I} i$ is the involvement time' which incorporates recognition and handling of the prey item (Hughes 1979). In relation to the Ei/I $i$ ratio, inducible defenses decrease the 'profitability' of the prey by increasing the value of $\mathrm{I} i$, while E $i$ remains constant. A decrease in profitability is thought to enhance prey survival. However, some negative effects on growth can be incurred by the induction of anti-predator defenses. For example, growth rates and internal shell volume are generally inversely related to shell thickness (Hickman 1979, Cheung et al, 2004), and the allocation of energy to the production of more byssus, thicker adductor muscles and reduction of the gape of valves can negatively impact mussel growth rates(Hawkins and Bayne 1985).

The vulnerability of an individual prey to crab predation at any point is not only dependant on the 'profitability' of the individual prey item but also on the density of the species population and the amount of protection afforded by its environment (Blundon and Kennedy 1982, Côte 1995). For example farmed mussels may attract predators by existing in high concentrations of profitable prey species particularly as spat. Conversely living in dense aggregations often supplies individual mussels with protection from 
predators (Côte and Jelnikar 1999, Leonard et al. 1999). Farmed mussels experience more rapid growth than wild conspecifics because farms are located to optimize the environmental conditions required for mussel growth. As a result the shell thickness of a given farmed GSM is often significantly less than in a wild mussel of the same age. Thin shells will leave mussels more vulnerable to crab predation; however an increase in attachment strength and increased growth rates may counteract the vulnerability incurred by having thinner shells. Crab prey preferences are often set to some degree by prey size, at some point the prey item will become so large that the handling costs will start to outweigh the energetic benefits gained from the prey item. The rapid growth rate of farmed mussels could lead them to quickly reach a 'size refuge'; a size at which molluscivorous crabs prefer not to, or are not able to consume them (Brousseau et al. 2001, Cheung et al. 2004).

N. minor is a common crab that is often encountered on GSM farms in the Marlborough Sounds (pers. obs, pers. comm. Kevin Heasman, Cawthron Institute, Nelson (2007), pers comm. Dan McCall, Marlborough Mussels Ltd., Nelson (2007) pers comm. Aaron Pannell, Marlborough Mussels Ltd., Blenheim (2007), (Clarkson et al. 2003)). Although mussels may only constitute a small portion of the diet of these crabs, the feeding behaviour, movement and abundance of $N$. minor may significantly affect the survival and growth of individual GSMs on the farm lines. Any loss of individual mussels or reduction in mussel growth will have flow on effects to the mussel farmer due to changes in the value and economic viability of the mussels. Because the interaction between $N$. minor and farmed GSM has not been investigated previously and the potential for economic losses exists it is crucial to understand the relationship between $N$. minor abundance and behavior and GSM responses. 


\subsection{CURRENT STUDY: OUTLINE AND AIMS}

N. minor is a common fouling organism on GSM farms in the Marlborough Sounds. Anecdotal evidence from individuals working in the aquaculture sector suggests that these crabs can cause significant GSM spat losses and increase mussel attachment rates, however no published data are available to substantiate these claims. The focus of the present study is on the potential financial losses incurred by GSM farmers due to $N$. minor presence, however ecological implications will also be discussed. The indirect and direct effects of $N$. minor presence on GSM spat will be investigated; particular consideration will be given to the following points:

1) The effect of $N$. minor presence on the retention and productivity of GSM spat in the field. Specific consideration is given to predation pressure and induced antipredator defenses, both of which can cause financial losses to mussel farmers;

2) The effects of $N$. minor density, size and sex when feeding on GSM. The growth rates of GSMs were investigated in the presence of $N$. minor, and the possibility of the existence of size refuges for the prey species are discussed; and

3) Whether the presence of $N$. minor can cause significant increases in the attachment strength of GSMs and whether $N$. minor presence has an effect on the thickness and number of byssus threads produced by GSM. The economic consequences of changes in byssus characteristics and GSM attachment strength are also discussed.

This study incorporates field experimental assessment of predation and fouling rates on locally caught GSM spat and Kaitaia spat in Pelorus Sound, Marlborough Sounds, New Zealand. Crab size and sex effects were investigated in a laboratory setting using wild caught $N$. minor and hatchery raised GSM. 
Due to the structure of this document there is some repetition of the core ecological theories and context, regarding the direct and indirect effects of the predatorprey interaction between $N$. minor and GSM. 


\section{Chapter 2: Predator induced changes in Greenshell mussel byssal characteristics and attachment strength.}

\subsection{INTRODUCTION}

An inducible defence can be defined as a phenotypical response to external cues that offers some protection from biotic selective agents such as predators (Adler and Harvell, 1990; Clark and Harvell, 1992; Freeman, 2007). Examples of induced defences in marine systems have been observed for bryozoans (Harvell 1984) barnacles (Lively, 1986), gastropods (Trussell, 1996), algae (Lampert et al, 1994) and bivalves (Freeman, 2007; Leonard et al., 1999; Reimer and Tedengren, 1996, Reimer and Harms-Ringdahl, 2001; Smith and Jennings, 2000). Examples of induced defences include: the production of spines, keels, and helmets in marine and freshwater invertebrates, changes to shell

allometry (Côte 1995), increases in the attachment strength in sessile bivalves (Lin, 1991), and changes in the level of 'defence' chemicals in terrestrial plants (Baldwin 1988). As outlined above, examples of the individual short-term benefits of inducible are numerous, however the relationship between induced defenses and long-term population dynamics has largely been overlooked. From an evolutionary perspective the benefits of inducible defenses have to be balanced by a fitness cost; if no costs were involved there would be no reason for a defense to be induced intermittently rather than be present constantly (Harvell 1990). The fitness costs can include reductions in growth, reproductive output and timing and reduced survivorship (Harvell 1990).

The development of inducible defenses in marine bivalves occurs more often in small less mobile species that are particularly vulnerable to predation (Bertness and Cunningham 1981). Many mobile molluses have also developed predator-specific 
avoidance and escape mechanisms. For example, the New Zealand scallop (Pecten noveazelandiae) uses a swimming response to escape from invertebrate predators (Allen 1997). The sea scallop Placopecten magellanicus (Gmelin) also uses swimming as an effective escape mechanism in response to sea stars, however this swimming response does not occur when the sea scallops are exposed to predatory crabs (Wong and Barbeau, 2004).

Most bivalve molluscs are sessile, living attached to a solid substrate or semiburied in sandy substrata, and hence lack the ability to physically evade predation (Côte 1995). Alternative methods of protection against predators have developed including: changes to shell allometry, increased shell thickness, living in dense aggregations, and gape reduction (Elner and Hughes 1978, Blundon and Kennedy 1982, Smallegange and van der Meer 2003, Cheung et al. 2004). These traits reduce predation mortality by increasing predator handling time which in turn reduces prey profitability and the energy gained from the prey item (Côte 1995).

Sessile bivalves often also attach more securely to, or bury more deeply into, the substrate when predators are present (Blundon and Kennedy, 1982, Côte, 1995, Lin, 1991). Like many other mussel species, the Greenshell ${ }^{\mathrm{TM}}$ mussel, Perna canaliculus (hereafter GSM) uses byssus threads to attach to substrata (Carrington, 2002, Jeffs et al., 1999). Byssus threads are not produced continuously but are produced, with a lag time, in response to various environmental stimuli (Côte 1995) including: wave action, (Young 1985, Dolmer 1998, Carrington 2002) changes in salinity (Young 1985)), temperature (Christophersen and Strand 2003, Carton et al. 2007) oxygen concentration (Alfaro 2005) and structural instability (Alfaro 2005). Bell and Gosline (1996) established that byssus thread number is the primary determinant of attachment strength. 
Byssus thread number and diameter increase in the presence of predatory crabs and crab effluent (Côte 1995, Reimer and Tedengren 1996, Leonard et al. 1999). Crabs will generally need to manipulate a prey item extensively before consumption, and not being able to remove a prey item from the substrate will greatly reduce its attractiveness to crabs. Published data regarding the plasticity of byssus thread production support the hypothesis that mussels will produce more and thicker byssus threads in the presence of invertebrate predators, and that this is a valuable anti-predator response.

In this study the focal bivalve species, the endemic New Zealand GSM, is one of the most extensively farmed marine species in New Zealand waters, and the potential predator, the camouflage crab Notomithrax minor, commonly occurs on Greenshell ${ }^{\mathrm{TM}}$ mussel farms in the Marlborough Sounds. This study will investigate GSM byssogenesis, attachment strength and changes to byssus thickness in the presence of the omnivorous crab Notomithrax minor, in the Marlborough Sounds, New Zealand. Anecdotal evidence from individuals working in the aquaculture sector suggest that $N$. minor could increase mussel attachment rates, however no published data are available to substantiate these claims. Increased mussel attachment strength could contribute to understanding and reducing one of the main problems faced by the industry, extremely low spat retention. Only about $2 \%$ of all mussel spat applied to grow-out ropes remain and grow to a harvestable size (Webb and Heasman 2006). Increased byssal attachment rates might increase the retention rates induce of newly seeded GSM spat; any factor that could lead to even a slight increase in spat retention levels could save the mussel industry hundreds of thousands of dollars annually. 


\subsection{METHODS}

\subsubsection{Pelorus Sound}

Pelorus Sound is one of the longest in the Marlborough Sounds, stretching $35 \mathrm{~km}$ from the Pelorus River at Havelock in the south ('inner' Sound), to the Cook Strait in the north ('outer' Sound). Pelorus Sound has several major arms including Tawhitinui Reach and Kenepuru Sound.

Hickman et al. (1991) sampled 6 sites in Pelorus Sound for salinity, total particulates, Chl $a$ and suspensoids on a bimonthly basis over two years (1983-1985). Salinity varied from 29-34\%o across the Sound with an increase in the inner and middle regions, and remaining relatively high and steady in the outer Sound. Water temperatures followed a similar trend with the outer Sounds being more stable; the water temperature in the outer Sounds was higher in the winter and lower in the summer with the inner sounds following an inverse trend (Fig 2.1). Chl $a$ varied between sites in the Sound, however seasonal patterns within and between sites were not evident. Values varied between $0.3-3.2 \mathrm{mg} \cdot \mathrm{m}^{-3}$ with the highest values constrained to the inner Sound due to influx from the Pelorus River.

Table 2.1: Means and ranges (in brackets) of suspended solids, Chl $a$ and Particulate Carbon measured in Pelorus Sound, Jan. 1984-Apr. 1985. Current study sites Laverique Bay and Crail Bay fall into the 'Middle' region. (Adapted from (Gibbs et al. 1992)

\begin{tabular}{l|ccc}
\hline REGION & SUSPENDED SOLIDS $\left(M G . M^{3}\right)$ & $\begin{array}{l}\text { CHLORPHYLL A } \\
\left(M G . M^{3}\right)\end{array}$ & $\begin{array}{l}\text { PARTICULATE CARBON } \\
\left(M G . M^{3}\right)\end{array}$ \\
\hline INNER & $1139(372-3630)$ & $1.81(0.6-6.0)$ & $327(46-1472)$ \\
\hline MIDDLE & $1002(196-6320)$ & $1.39(0.16-4.4)$ & $247(67-600)$ \\
\hline OUTER & $778(129-2310)$ & $1.31(0.13-4.7)$ & $213(43-529)$ \\
\hline
\end{tabular}




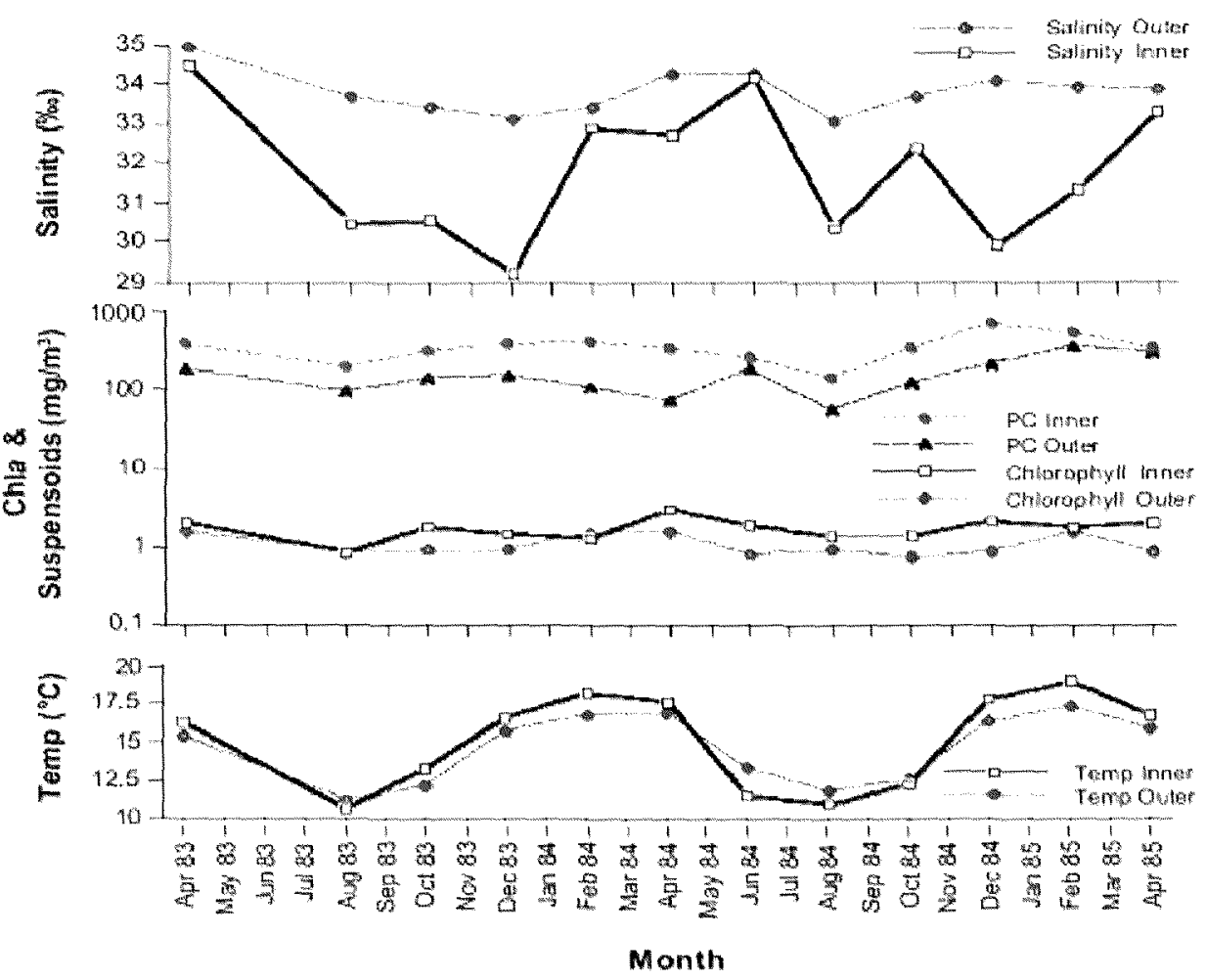

Figure 2.1: Mean readings of salinity, water temperature, Chl $a$ and suspensoids for an inner and an outer Sound (relative to the ocean) site in the Pelorus Sound, New Zealand. Modified from (Hickman et al. 1991a)

(Gibbs et al. 1992) examined particulate and Chl $a$ flux between sites in Pelorus Sound. Significant levels of particulate and $\mathrm{Chl} a$ variability were apparent (Table 2.1). These were attributed to the complex hydrodynamic regime that includes three major nutrient sources: sediment remineralisation, transfer from the ocean and river inflows.

\subsubsection{Laverique Bay and Crail Bay}

During the two study periods, winter 2006 (June-September) and summer 2006/2007 (November-January), caging experiments were carried out on GSM spat farms in Pelorus Sound, the Marlborough Sounds, New Zealand. The farms were located at two discrete sites within Pelorus Sound (Laverique Bay and Crail Bay). The sites are common spat catching and grow-out sites, selected for their water quality, phytoplankton 
concentrations and relatively low density of predatory fish. Both sites are located in Beatrix Bay, in eastern Pelorus Sound.

Both study sites are situated in a large eastern branch of the Pelorus Sound. The branch contains three major bays: Beatrix, Crail and Clova Bay, all of which support a large number of marine farms (Fig. 2.2) Site 1 (Laverique Bay $41^{\circ} 01^{\prime} \mathrm{S}, 174^{\circ} 02^{\prime} \mathrm{E}$ ) is in the south eastern area of Beatrix bay and site 2 is on the north western shore of Crail Bay $\left(41^{\circ} 05^{\prime} 393 \mathrm{~S}, 173^{\circ} 58^{\prime} 205 \mathrm{E}\right)$ (Fig. 2.2).

Table 2.2: Site specific temperature, salinity and chlorophyll a measurements for Laverique Bay and Crail Bay. The data is from 2005-2006, for the summer and winter periods during which current testing took place.

\begin{tabular}{|c|c|c|c|c|c|c|}
\hline \multirow[t]{2}{*}{ WEEK } & \multicolumn{2}{|c|}{ TEMPERATURE $\left({ }^{\circ} \mathrm{C}\right)$} & \multicolumn{2}{|c|}{ SALINITY (PPM) } & \multicolumn{2}{|c|}{ CHL A ( $\mu G$. LITRE $\left.{ }^{-1}\right)$} \\
\hline & $\begin{array}{c}\text { LAVERIQUE } \\
\text { BAY }\end{array}$ & CRAIL BAY & $\begin{array}{c}\text { LAVERIQUE } \\
\text { BAY }\end{array}$ & CRAL BAY & $\begin{array}{c}\text { LAVERIQUE } \\
\text { BAY }\end{array}$ & CRAIL BAY \\
\hline DEC 04-DEC10 & 16 & 17.5 & 33.5 & 33.3 & $0.1-1.2$ & $0.1-1.3$ \\
\hline DEC 18-DEC 25 & 17.5 & 18 & 34 & 34 & $0.1-0.3$ & $0.1-1.5$ \\
\hline JUN 12-JUN 18 & 12.5 & 12.7 & 33.2 & 33.8 & $0.8-1.2$ & $0.9-1.2$ \\
\hline JUN 26-JUL 02 & 11.7 & 12.0 & 33.6 & 33.9 & $1.0-1.7$ & $0.8-1.2$ \\
\hline
\end{tabular}

Data adapted from NIWA 'Marlborough Sounds Environmental Monitoring Programme' (2005-2006).

The Crail Bay study site is situated in relatively deep water (approx 30m), whereas the Laverique Bay study site is around $25 \mathrm{~m}$ deep. Both sites are moderately exposed to wind and water movement, they also have similar salinity, temperature and Chl $a$ characteristics (Table 2.2).

\subsubsection{Experimental design}

Cages were placed on droppers at both sites to retain experimental crabs within a specific area of spat line. The cages used were commercial shellfish grow-out apparatus called Aquapurses. The specific Aquapurses used were $0.8 \mathrm{~m}$ long with $8 \mathrm{~mm}$ mesh; a $20 \times 20 \mathrm{~mm}$ hole was cut in the top and bottom of each cage to allow the cage to be 
attached around the mussel line. Aquapurses are manufactured by Tooltech Pty Ltd (Australia) and are used commercially in oyster culture in NZ.

Long-lines in the interior of the farms were selected to minimize the variation of exposure among experimental droppers. On the target long-line, top and bottom locations were avoided: cages were placed at $4 \mathrm{~m}$ depth on randomly selected $8 \mathrm{~m}$ long single droppers, one cage per dropper. At Laverique Bay, the farm is set-up using single droppers which consist of $8 \mathrm{~m}$ lengths of commercial spat catching rope, commonly known as Christmas tree rope. Cages were placed on the northwest side of the backbone at $4 \mathrm{~m}$ depth, but on one dropper the cage was placed shallower $(3 \mathrm{~m})$, because of scuffing during earlier movement of the spat lines which had removed most of the mussels at the desired depth. At Crail Bay, continuous $12 \mathrm{~m}$ long droppers were used and single cages were placed on droppers on the east side of the backbone (Fig. 2.3).

The droppers at Laverique Bay were placed in the water on the $8^{\text {th }}$ of March 2006 to allow mussel spat to settle naturally on the lines. The field trial commenced on the $25^{\text {th }}$ of July 2006 and by this time the spat had an average total shell length (TSL measured across the anterior dorsal-posterior ventral axis) of $16.8 \mathrm{~mm} \pm 0.79(95 \%$ C.I.). GSM density was considerably higher in the uppermost $1 \mathrm{~m}$ of the droppers. Upon visual inspection at commencement of the field trial there was no substantial "oversettlement' of $M$. galloprovincialis, although numerous other species were also present on the lines, including various scallop species, bryozoans, algae and a small number of decorator crabs between $10-20 \mathrm{~mm}$ total carapace width (TCW measured at the widest point of carapace). 


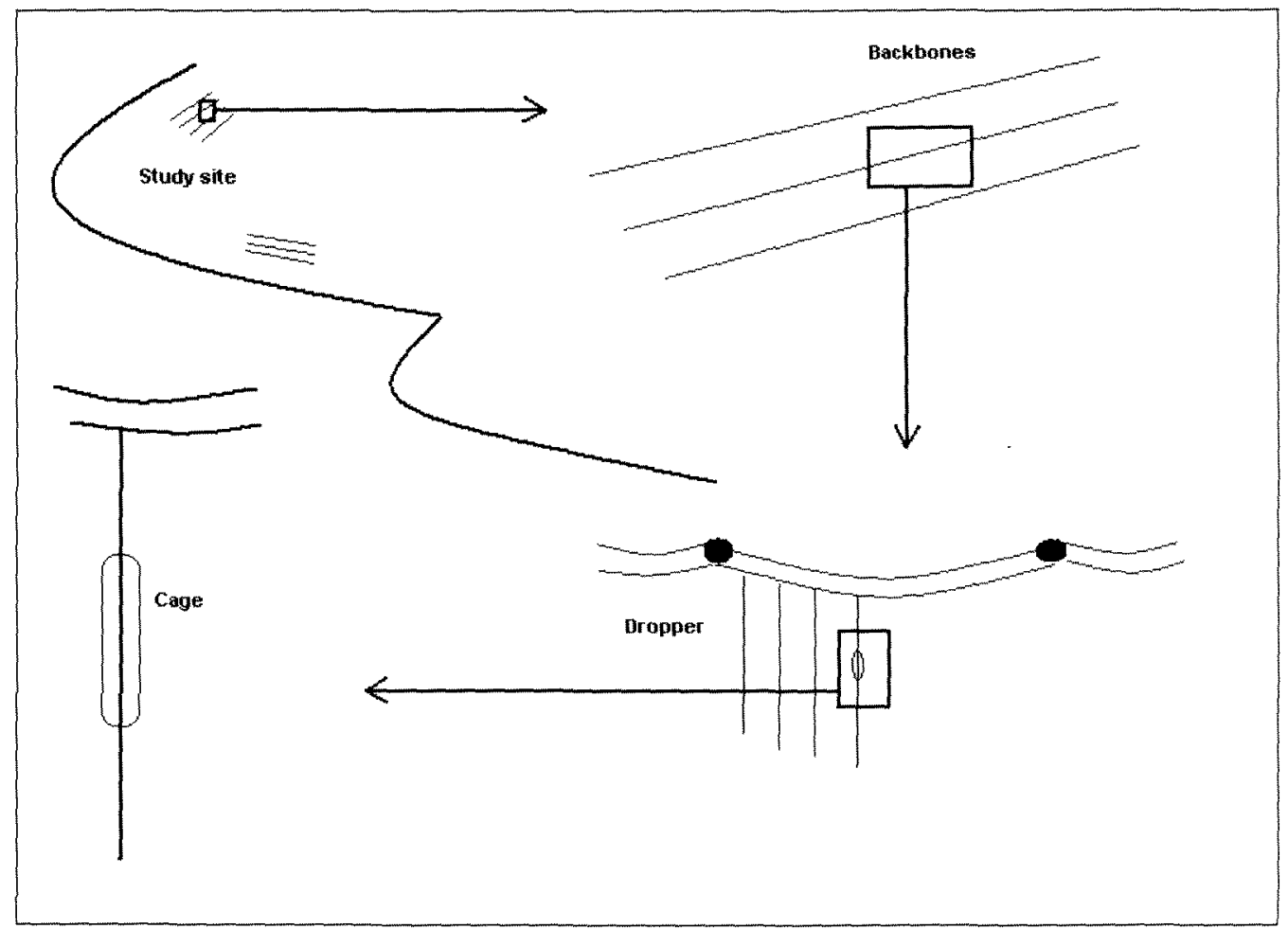

Figure 2.2: schematic illustration of the construction of a GSM mussel farm in the Marlborough Sounds, outlining terms in the methodology (study site, backbone, dropper and cage) and the positioning of the experimental cages.

The GSMs at Crail Bay were sourced from Kaitaia spat collected at Ninety Mile Beach and seeded mechanically onto the spat ropes $3 \mathrm{wks}$ before commencement of the field trial $\left(23^{\text {rd }}\right.$ November 2006). Due to the spat being seeded onto the line there was minimal over-settlement of other species; M. galloprovincialis were present in very low numbers. At the start of the field trial at Crail Bay the average size of the GSM spat was $4.86 \mathrm{~mm} \pm 0.56$ (95\% C.I.). Some cotton stocking, the material used to encase newly seeded spat until the mussels attach to the spat rope, was still visible but degraded to the extent that the mussels were readily accessible to predators. 


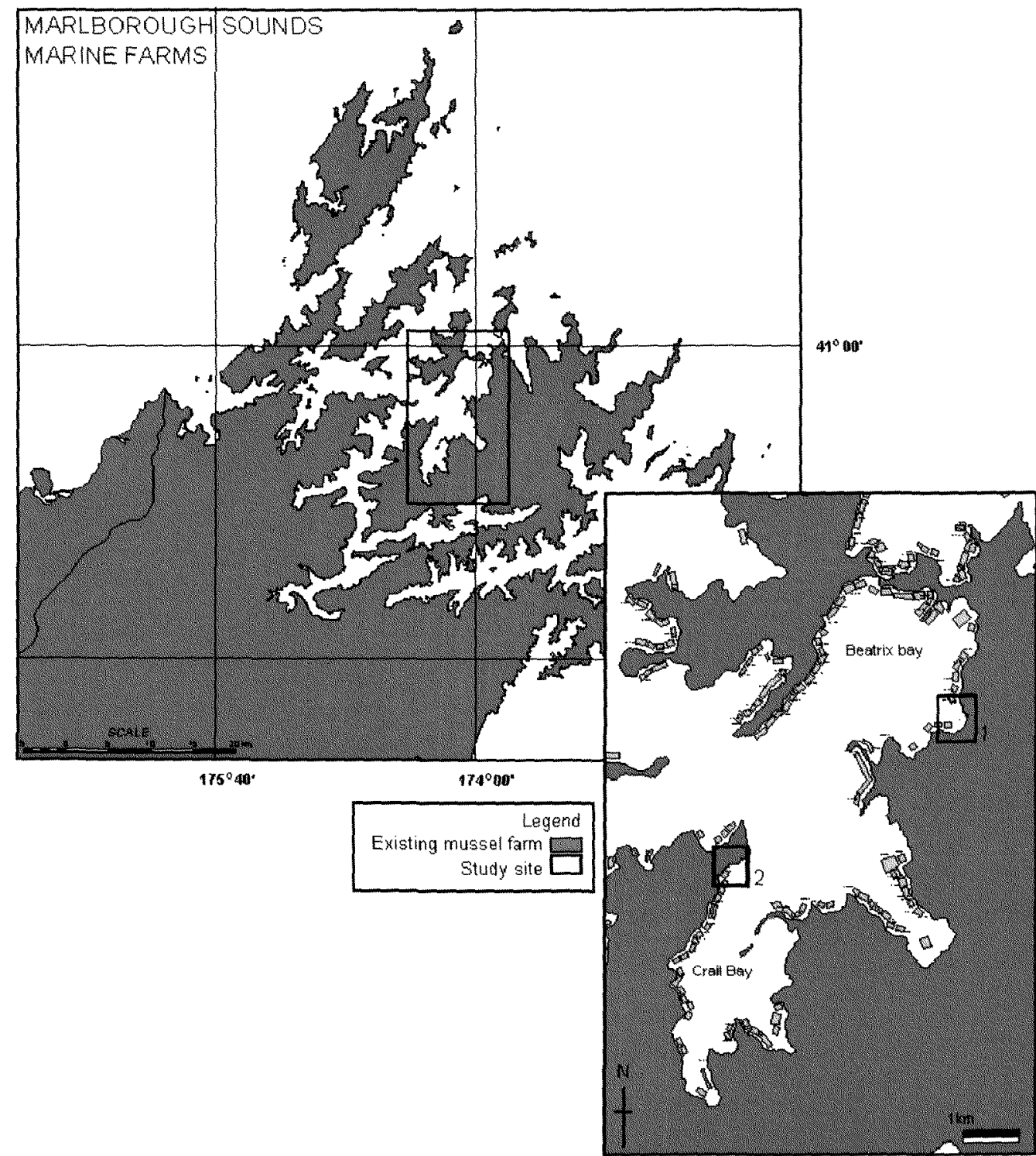

Figure 2.3: The location of the study sites. Boxes indicate site locations within Pelorus Sound, Crail Bay to the south (2) and Laverique Bay to the north (1). Locations of existing mussel farms are from Marlborough District Council data (2003).

The experimental crabs were collected from existing mussel farms in the Marlborough Sounds 10 days before commencement of the field trials and held in large ( $1.3 \mathrm{~m}$ width $\times 1.3 \mathrm{~m}$ height $\times 0.75 \mathrm{~m}$ depth) black plastic tanks in the laboratory at the Cawthron Aquaculture Facility, Nelson. Crabs were fed to excess $24 \mathrm{hr}$ after collection with fresh GSM flesh. $24 \mathrm{hr}$ before onset of the field trial the crabs were sorted into the 
relevant treatments (detailed below) and weighed to $0.1 \mathrm{~g}$, to allow estimation of crab growth over the experimental period. Crabs were transported to each site in a $90 \mathrm{~L}$ bin filled with fresh seawater with two battery powered air pumps oxygenating the water. The caged section of mussel rope was checked for any local crabs, which were removed before the experimental crabs were introduced.

The effects of crab density and size on GSM byssus thickness, byssus number and attachment strength, in the presence of different crab treatments were tested. Previous visual assessments and anecdotal evidence from the GSM industry of $N$. minor density on existing GSM farms were used to define crab density and size treatments. Treatments included: high $(\mathrm{H})$ (12 crabs) and average density (L) (3 crabs), of both large (La) and medium sized crabs (M). Large males had TCW $>20 \mathrm{~mm}$, large females were $>15 \mathrm{~mm}$, medium males were $15-20 \mathrm{~mm}$, and medium females were $10-15 \mathrm{~mm}$ TCW. Sex ratio in the cages was haphazardly selected. A zero density caged treatment (CC) and an un-caged control (UC) were also included in the trails to assess caging effects. Only medium and large crabs were used because of the cryptic nature of these crabs and the logistic difficulty involved with the collection of small individuals. At Laverique Bay, 3 replicates of each treatment were retrieved after $6 \mathrm{wk}$ and again after $11 \mathrm{wk}$, At Crail Bay, 8 replicates of each treatment were retrieved after $8 \mathrm{wk}$ (Table 3.2). The differences in methodology for the two study sites was due to the fact commercial GSM spat were used, thus experimental design was limited by the quantity and type of spat made available by commercial GSM farmers. Optimally the entire Laverique Bay trial would have been run for 11 weeks due to it being run during the winter. Predation rates and GSM growth rates are generally lower during the winter months. However the GSM spat at Laverique Bay needed to be harvested for on growing after 6 weeks, only half of the 
spat was made available for longer term experimentation (11 wk). The Crail Bay trial was run for a shorter period due to the fact it was run in the summer months and no limitations were placed on experimental design by the GSM farmers at this study site.

Table 3.2: Detailed outline of experimental design at Laverique Bay and Crail Bay

\begin{tabular}{|c|c|c|c|c|c|}
\hline \multirow[t]{2}{*}{ Treatment } & \multirow[t]{2}{*}{ Crab Size } & \multicolumn{2}{|c|}{ Crail Bay (Winter) } & \multicolumn{2}{|c|}{ Laverique Bay (Summer) } \\
\hline & & Put out & Retrieved & Put out & Retrieved \\
\hline \multirow{4}{*}{ High $(H)$} & \multirow{2}{*}{ Medium $(M)$} & $11 / 07 / 06$ & $22 / 08 / 06(6 w k)$ & \multirow{2}{*}{$23 / 11 / 06$} & \multirow{2}{*}{$18 / 01 / 07(8 w k)$} \\
\hline & & $11 / 07 / 06$ & $22 / 10 / 06(11 w k)$ & & \\
\hline & & $11 / 07 / 06$ & $22 / 08 / 06(6 w k)$ & \multirow{2}{*}{$23 / 11 / 06$} & \multirow{2}{*}{$18 / 01 / 07(8 w k)$} \\
\hline & Large (La) & $11 / 07 / 06$ & $22 / 10 / 06(11 w k)$ & & \\
\hline \multirow{4}{*}{ Average (A) } & & $11 / 07 / 06$ & $22 / 08 / 06$ (6 wk) & \multirow{2}{*}{$23 / 11 / 06$} & \multirow{2}{*}{$18 / 01 / 07(8 w k)$} \\
\hline & Medium $(M)$ & $11 / 07 / 06$ & $22 / 10 / 06$ (11wk) & & \\
\hline & \multirow[b]{2}{*}{ Large(La) } & $11 / 07 / 06$ & $22 / 08 / 06(6 w k)$ & \multirow{2}{*}{$23 / 11 / 06$} & \multirow{2}{*}{$18 / 01 / 07(8 w k)$} \\
\hline & & $11 / 07 / 06$ & $22 / 10 / 06(11 w k)$ & & \\
\hline \multirow[b]{2}{*}{ Zero } & \multirow[b]{2}{*}{ Caged $(C C)$} & $11 / 07 / 06$ & $22 / 08 / 06(6 w k)$ & \multirow{2}{*}{$23 / 11 / 06$} & \multirow{2}{*}{$18 / 01 / 07(8 w k)$} \\
\hline & & $11 / 07 / 06$ & $22 / 10 / 06(11 w k)$ & & \\
\hline \multirow[b]{2}{*}{ Uncaged } & \multirow[b]{2}{*}{ Control (UC) } & $11 / 07 / 06$ & $22 / 08 / 06(6 w k)$ & \multirow{2}{*}{$23 / 11 / 06$} & \multirow{2}{*}{$18 / 01 / 07(8 w k)$} \\
\hline & & $11 / 07 / 06$ & $22 / 10 / 06(11 w k)$ & & \\
\hline
\end{tabular}

\subsubsection{Data collection}

At each retrieval date (Table 3.2) the spat lines were lifted and the $0.8 \mathrm{~m}$ section inside the cage was removed. On uncaged control lines a $0.8 \mathrm{~m}$ section of line was cut at $4 \mathrm{~m}$ depth. The top and bottom $10 \mathrm{~cm}$ of the caged and uncaged samples were excluded due to clumping of mussels against the ends of the cages. At a randomly selected point on the caged (and un-caged control) section of line 20 mussels were removed with byssus threads intact. Once removed the mussels were placed in $100 \mathrm{ml}$ plastic containers containing freshwater, which causes GSM to seal their valves and hence production of additional byssus threads was avoided during transport.

The byssus was dissected away from the foot of the mussel and stored in freshwater until byssus measurements and counts could take place. The byssus threads 
were counted and measured using a compound microscope with an ocular micrometer. Two measurements and one count were performed for each byssus sample: 1) 'stem diameter' across the base of the byssus root, 2) 'byssus diameter' three byssus threads were measured at the base, the average of the three measurements was recorded. 3) 'byssus number' the total number of byssus threads were counted (Fig. 3.3).

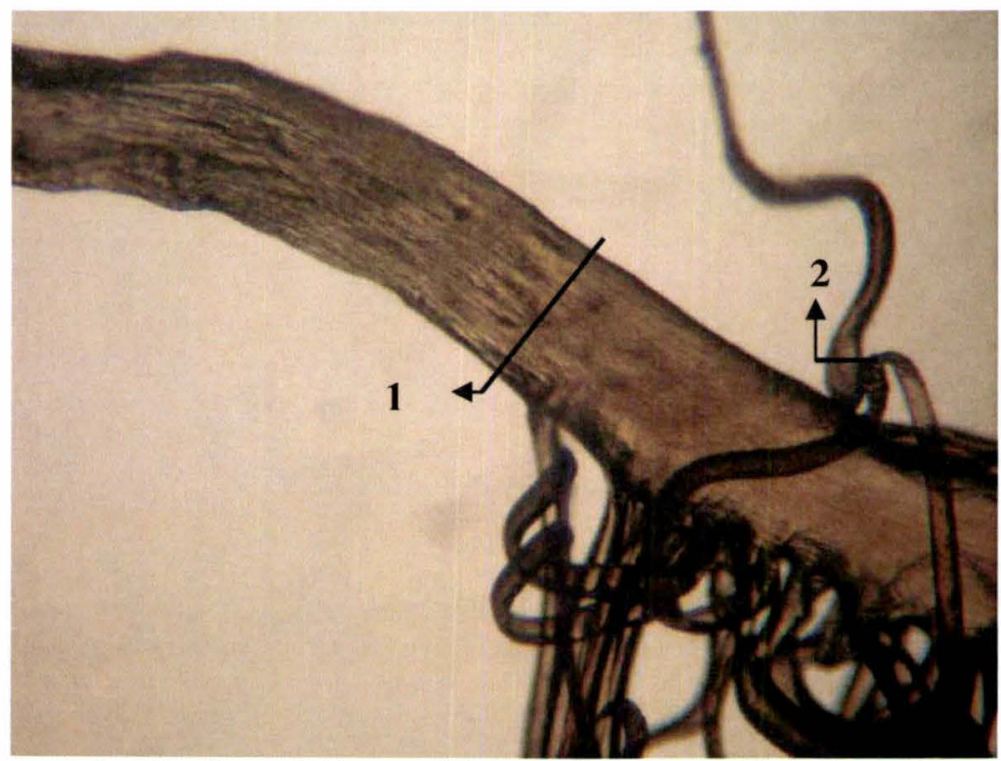

Figure 2.4: An example of a Greenshell mussel byssus thread magnified 40x, indicating the location of measurements recorded for the analyses. 1: 'stem' diameter, 2: 'byssus' diameter.

Attachment strength was measured for 10 mussels per dropper for all treatments for the second pick up date at Laverique Bay, and for all the droppers at the Crail Bay site. A spring loaded t-bar scale with a small hook attachment was used to assess attachment strength. The hook was placed between byssus threads at the base of the mussel, the mussel rope was held tightly and the scale drawn away from the mussel rope until the mussel detached. The weight in grams on the scale was read off and recorded at the time of detachment of the mussel from the rope. 


\subsubsection{Statistical analysis}

The statistical and data packages SPSS 14.0 for Windows (SPSS Incorporated, Chicago, Illinois) and SigmaPlot 9.01 for Windows (SyStat Software, Inc.) were used for graphing and analysis of the data.

The total and average byssus thread number, average 'stem' diameter ( $\mathrm{mm}$ ) and 'byssus' diameter $(\mathrm{mm})$ were calculated for each sample. The averages for the three byssus variables (byssus number, 'stem' diameter and 'byssus' diameter) were also calculated for high and low crab density and for the two crab size classes (large and medium) at Laverique Bay and Crail Bay.

Cochran's Q test was used to assess the equality of variances across the crab density, size and control treatments, and Normal $Q-Q$ plots were constructed to test the normality of the data. Any outliers were checked against the raw data and if they were genuine outliers, the data points were deleted. Byssus thread number data were transformed $\left(\log _{10}\right)$ to meet the requirements of Analysis of Variance (ANOVA), 'byssus' and 'stem' diameter data met the assumptions of ANOVA and were not transformed.

The data for stem and byssus diameter, byssus thread number and attachment strength were standardized for days by dividing the values by the number of days the trial ran for, to make comparisons of data from experiments that ran for different lengths of time viable. After standardization the data were categorized into two data sets: Laverique Bay and Crail Bay. The standardized data were subjected to ANOVA tests to test the null hypothesis $\left(H_{0}\right)$ that crab $(N$. minor $)$ density and size will have no effect on the number and diameter of the byssus threads produced by GSM spat. Due to the fact the crabs consumed all the mussels in the high crab density treatments at Crail Bay the 
model was not totally balanced, hence two one-way ANOVA models were run at this site. One for crab density and one for crab size. At Laverique bay there were mussels present in all replicates so a 2-way ANOVA model was used at this site. In Both the one-way and 2-way ANOVA model crab density and crab size were considered to be fixed factors. An ANOVA test was also run to assess the differences in byssus thread number and thickness between the two study sites, in this model the study sites were random factors. ANOVA is a robust test that is suitable to use when analysing ecological data. Any results representing significant differences at $\alpha=0.05$ were subjected to post hoc Tukey tests (pair wise comparisons) to establish the locations of the significant differences. Due to the prioritization of the data collection, mussels were counted to assess predation, then 20 were removed as outlined above to assess byssus characteristics. In many of the 'crab present' treatments there were less than the required 20 mussels left to test attachment strength, leaving the dataset unbalanced at both study sites. Two one-way ANOVA tests were run on the attachment strength data due to the lack of data in some treatments; hence the interaction between crab density and crab size was not assessed. Separate ANOVA analyses were chosen over MANOVA tests because the response variables were significantly correlated (Pearson and Kendall's tau-b tests in SPSS).

A multiple linear regression model analysis was also run on the standardized data in SPSS to asses the collective and individual extent to which the three byssus test variables ('byssus' thickness, 'stem' thickness and byssus number) affect the attachment strength of GSMs at Crail Bay and Laverique bay. Normal $Q-Q$ plots were used to assess the normality of the data, and an intercept of 0 was included to account for other environmental factors such as temperature and water flow that weren't included in the 
analysis and could also be affecting byssus characteristics. Attachment strength data, byssus number, byssus thread and stem thickness were all $\log _{10}$ transformed to increase the normality of the data for both Crail Bay and Laverique Bay. In the linear regression model $(y$ (attachment strength $)=\beta_{0}+\beta_{1}$ (stem thickness $)+\beta_{2}$ (byssus thickness $)+$ $\beta_{3}$ (byssus number) $+\varepsilon i$ ) attachment strength was the dependant variable and byssus number, byssus width and stem width were independent variables. Colinearity was assessed prior to analysis, diagnostic checks of the model did not reveal any outliers and boxplots of the residuals were relatively symmetrical. Stepwise selection was used to elect the variables to include in the multiple regression model, the criteria for inclusion of a variable in the analysis was that the probability of $\mathrm{F}<=0.05$ and the probability of $\mathrm{F}$ to remove the variable $>=0.100$. The most statistically significant relationship between the dependant variable (attachment strength) and the independent variables (byssus number, byssus width and stem width) was identified. only the most significant linear regression was displayed in the results section. The original un-standardized data are presented in the graphs.

\subsection{RESULTS}

This section will give an overview of the results used to test the null hypothesis that decorator crabs (N. minor) will in no way affect the thickness and number of byssus threads produced by GSM spat in the Marlborough Sounds. This section is organized into crab density and crab size, these are the two factors used to evaluate the extent to which $N$. minor affects byssal stem thickness, byssus thickness and number. The final subsection will display the results of regression analyses run to investigate which of the 
aforementioned byssus characteristics could be the primary determinant of attachment strength.

\subsection{Crab density effects.}

Byssus thread thickness and stem thickness, byssus number and attachment strength of GSM spat showed a varied response to crab densities but also at both of the study sites; Crail Bay (CB) and Laverique Bay (LB). Byssus thickness was not significantly affected by variation in crab density at either study site (Table 2.3). Crab density also had no significant effect on the attachment strength of GSM spat at either study site (Table 2.3)

Increased crab density did stimulate GSM spat to produce thicker byssal stems at both sites (Fig 2.4, Table 2.3). Stem thickness peaked at high crab densities (12 crabs). ANOVA tests specify that the differences in stem thickness related to crab density were statistically significant at the $95 \%$ level at Crail Bay, but not for the mussel spat at Laverique Bay (Table 2.3).

A post hoc Tukey test of 'stem' thickness specified that at Crail Bay the differences across the four crab density treatments were significant except for the uncaged and caged control treatments $(p=0.755)$. The remaining pair wise comparisons all had a p-value of $<0.001$ (Fig 2.5).

Byssus thread number peaked in the high crab density treatments at both sites (Fig. 2.5). Post hoc Tukey test results supported the trends in byssus thread number seen in figure 2.5b; at Crail Bay there were statistically significant differences between all treatments $(p=<0.001)$ except between the average and high crab densities $(p=0.862)$. At Laverique Bay the differences in byssus thread number were not significantly 
different between the un-caged and caged control $(p=0.603)$, or between the un-caged control and the average crab density treatments $(p=0.759)$. The differences in byssus thread number between the remaining treatments were all significant at the $95 \%$ level. However the overall byssus thread number was higher for all treatments at Crail Bay than at Laverique Bay (Fig 2.5)
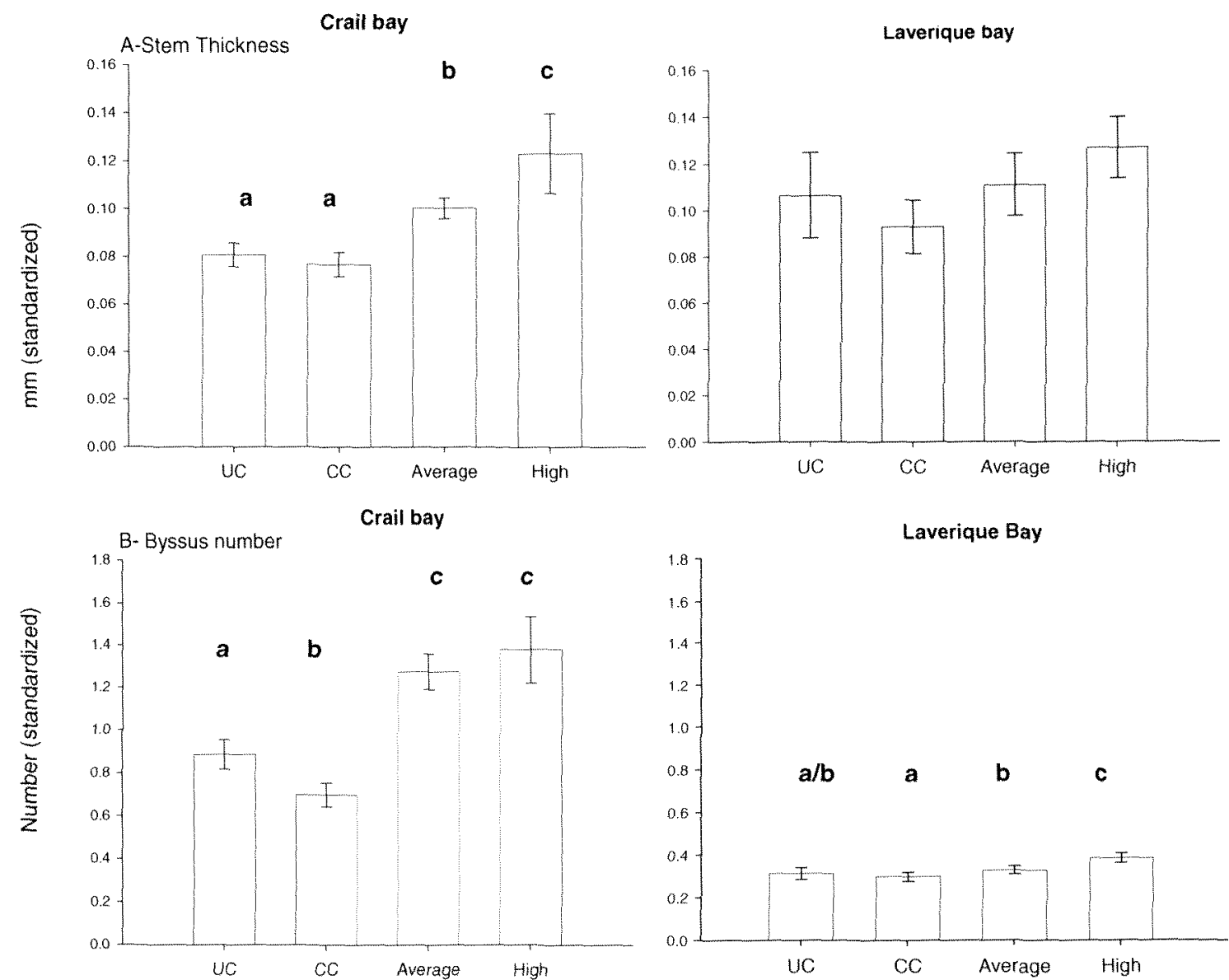

Figure 2.5: Effects of potential predator (N. minor) density on Greenshell mussel byssal stem thickness (mean $\pm 95 \%$ C.I.) and byssus number at the two study sites, Crail Bay and Laverique Bay. Stem thickness and Byssus number were standardized to days by dividing the total number by the number of days the experiment ran for 42 and 77 days at Laverique Bay, 56 days for Crail bay). At Crail bay the caged and un-caged controls had significantly thinner byssal stems and lower byssus numbers than the average and high crab density treatments. The differences in stem thickness and byssus number were not statistically significant at Laverique bay (ANOVA). Letters represent statistically significant post hoc groupings calculated using Tukey tests and ANOVA analysis, only Tukey test results for significant ANOVA results are displayed. Crab density treatments are as follows: UC- un-caged control, $\mathrm{CC}$ - caged control, Average- 3 crabs per cage, High- 12 crabs per cage. 


\section{Crab size effects}

Crab size had similar effects on stem thickness, byssus thickness and byssus number as crab density; the only major point of difference was in attachment strength.

Crab size did not have a statistically significant effect on byssus thickness at either Crail Bay (Table 2.3) or Laverique Bay. Stem thickness did change with crab size; mussels in the large crab treatments have the thickest byssal stems at both study sites ( (Fig. 2.6). At both sites these differences in stem thickness were not statistically significant at the $95 \%$ level (Table 2.3 ).

Table 2.3: ANOVA table for byssus characteristics (thickness and number) and attachment strength for both study sites Laverique Bay (LB) and Crail Bay (CB). Crab density and Crab size were considered fixed factors in the analysis and byssus number and attachment strength were $\log _{10}$ transformed to meet the assumptions of the analysis. Significant results are indicated in bold

\begin{tabular}{|c|c|c|c|c|c|c|c|c|c|}
\hline & \multirow[t]{2}{*}{ FACTOR } & \multicolumn{2}{|c|}{ DF. } & \multicolumn{2}{|r|}{ MS } & \multicolumn{2}{|c|}{$F$} & \multicolumn{2}{|c|}{ P-VALUE } \\
\hline & & LB & $\mathrm{CB}$ & LB & $\mathrm{CB}$ & LB & $\mathrm{CB}$ & LB & $\mathrm{CB}$ \\
\hline \multirow{4}{*}{ CRAB DENSITY } & $\begin{array}{c}\text { BYSSUS } \\
\text { THICKNESS }\end{array}$ & 1 & 1 & $\begin{array}{l}3.38 E- \\
005\end{array}$ & $\begin{array}{c}3.64 E- \\
009\end{array}$ & 0.855 & $<0.001$ & 0.356 & 0.990 \\
\hline & $\begin{array}{c}\text { STEM } \\
\text { THICKNESS }\end{array}$ & 1 & 1 & 0.016 & 0.006 & 2.982 & 13.738 & 0.760 & $<0.001$ \\
\hline & $\begin{array}{l}\text { BYSSUS } \\
\text { NUMBER }\end{array}$ & 1 & 1 & 0.185 & 0.091 & 7.434 & 5.578 & 0.556 & 0.019 \\
\hline & $\begin{array}{l}\text { ATTACHMENT } \\
\text { STRENGTH }\end{array}$ & 1 & 1 & 0.035 & 0.023 & 0.420 & 0.319 & 0.518 & 0.573 \\
\hline \multirow{4}{*}{ CRAB SIZE } & $\begin{array}{c}\text { BYSSUS } \\
\text { THICKNESS }\end{array}$ & 1 & 1 & $\begin{array}{l}5.20 E- \\
005\end{array}$ & $\begin{array}{c}2.09 E- \\
005\end{array}$ & 1.316 & 0.986 & 0.252 & 0.322 \\
\hline & $\begin{array}{c}\text { STEM } \\
\text { THICKNESS }\end{array}$ & 1 & 1 & 0.004 & $\begin{array}{l}2.70 E- \\
005\end{array}$ & 0.723 & 0.060 & 0.396 & 0.807 \\
\hline & $\begin{array}{l}\text { BYSSUS } \\
\text { NUMBER }\end{array}$ & 1 & 1 & 0.359 & 0.101 & 14.422 & 6.204 & $<0.001$ & 0.014 \\
\hline & $\begin{array}{l}\text { ATTACHMENT } \\
\text { STRENGTH }\end{array}$ & 1 & 1 & 0.443 & 0.293 & 5.293 & 3.991 & 0.023 & 0.048 \\
\hline \multirow{3}{*}{$\begin{array}{l}\text { CRAB DENSITY* } \\
\text { CRAB SIZE }\end{array}$} & $\begin{array}{c}\text { BYSSUS } \\
\text { THICKNESS }\end{array}$ & 1 & - & $\begin{array}{c}3.26 \mathrm{E}- \\
005\end{array}$ & 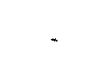 & 0.825 & - & 0.364 & - \\
\hline & $\begin{array}{c}\text { STEM } \\
\text { THICKNESS }\end{array}$ & 1 & - & $\begin{array}{l}2.13 \mathrm{E}- \\
006\end{array}$ & - & $<0.001$ & - & 0.984 & . \\
\hline & $\begin{array}{l}\text { BYSSUS } \\
\text { NUMBER }\end{array}$ & 1 & - & 0.125 & - & 5.019 & - & 0.026 & . \\
\hline
\end{tabular}


Byssus number and attachment strength were the only dependant variables that were significantly affected by crab size; mussels in the large and medium crab treatments had more byssus threads per mussel than both the control treatments at Crail Bay. And at Laverique bay the large sized crab treatments had more byssus threads per mussel than any of the other treatments. (Fig 2.5). Tukey tests supported the patterns observed in the graphs and ANOVA tests; at Crail Bay the two crab treatments (Medium and Large) had significantly higher numbers of byssus threads than the control treatments. At Laverique Bay the mussels in the large crab treatments had significantly higher numbers of byssus threads than all the other treatments (Fig. 2.6B, Table 2.3).

Attachment strength also varied significantly across crab size treatments at both Crail Bay and Laverique bay (Fig 2.5, Table 2.3). For both sites mussels in the control treatments (UC and CC) were less tightly attached than mussels in the two crab treatments (medium and large). Tukey tests also indicated that there were no significant differences in attachment strength between the two control treatments at Crail Bay $(p=0.129)$ or Laverique Bay $(p=0.363)$ or between the two crab treatments for (Crail Bay $p=0.221$, Laverique Bay $p=0.125)($ Fig 2.6) 

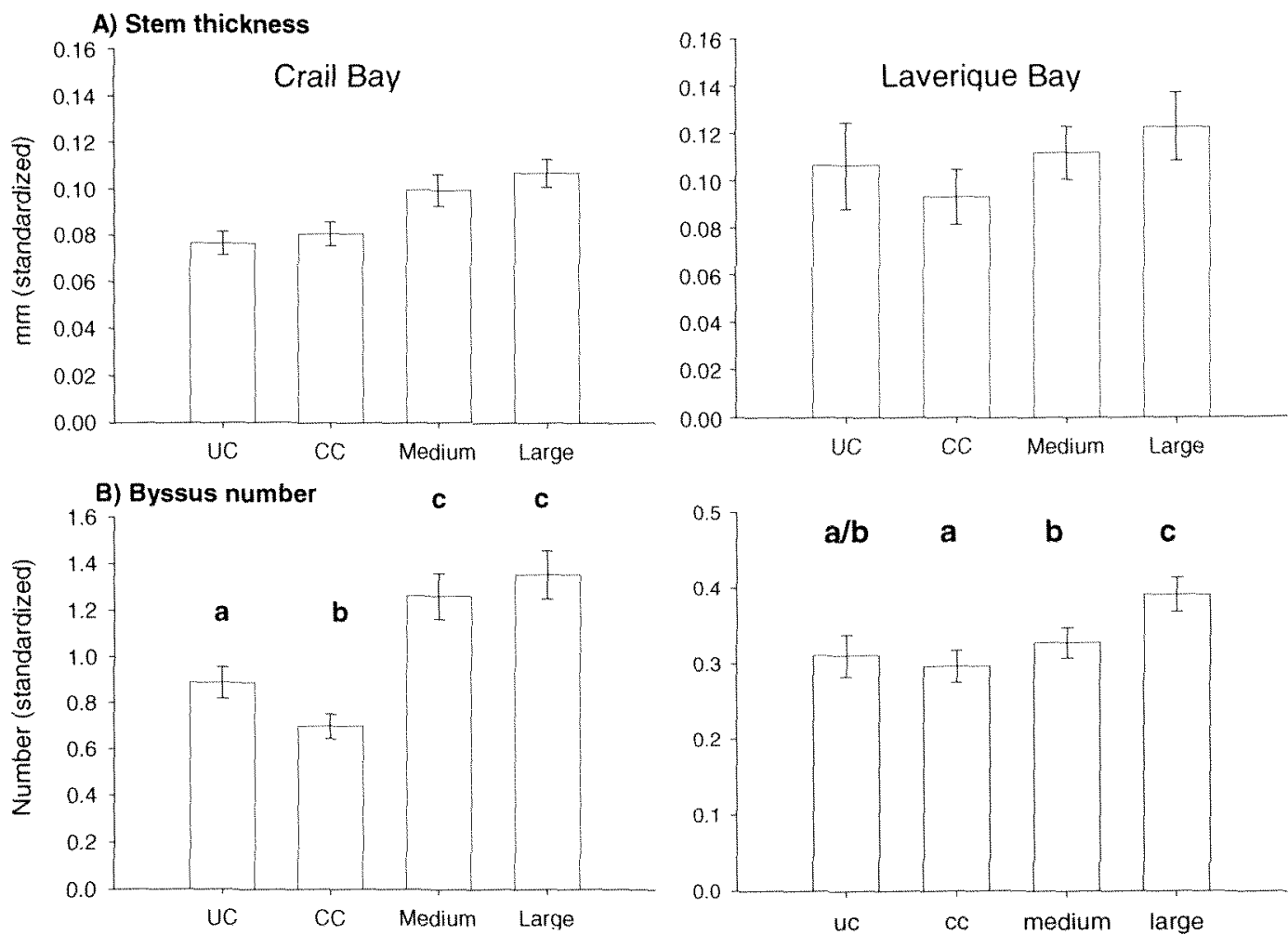

C) Attachment strength
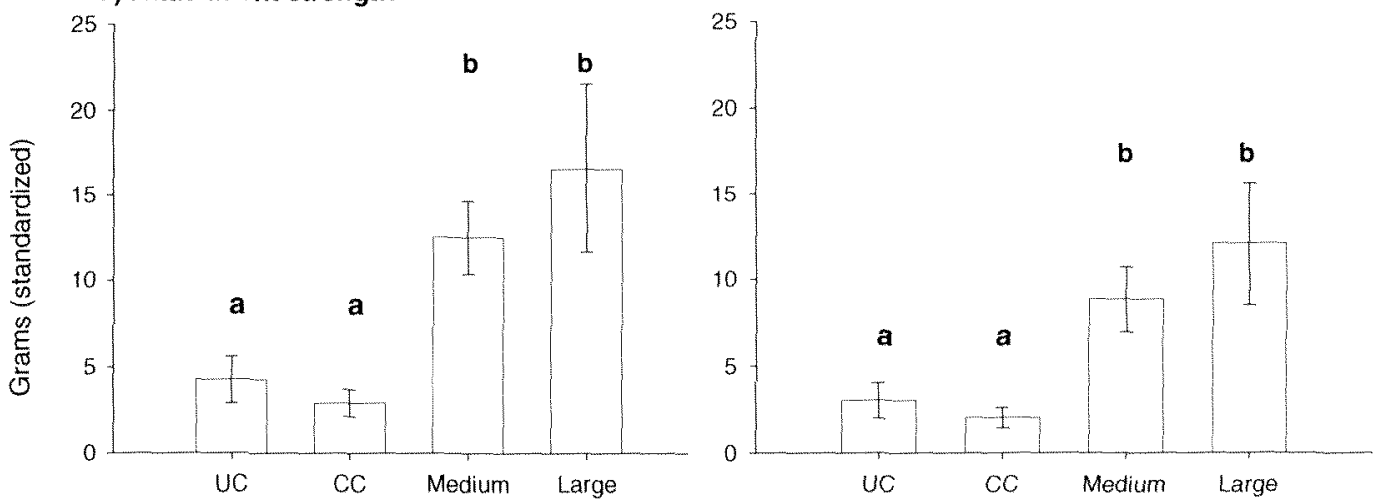

Figure 2.6: Effects of potential predator (N.minor) size on Greenshell mussel stem thickness, byssus number and attachment strength (mean $\pm 95 \%$ C.I.) at the two study sites, Crail bay and Laverique bay. Stem thickness, byssus number and attachment strength were standardized to days by dividing the total number by the number of days the experiment ran for ( 42 and 77 days at Laverique Bay, 56 days for Crail bay). At Crail bay the caged and un-caged controls had significantly thinner byssal stems and less byssus threads than the average and high crab density treatments. At both study sites mussels in the crab treatments (medium and large) had significantly higher attachment strengths than control mussels (CC and UC). Letters represent post hoc groupings calculated using Tukey tests, only Tukey test results for significant ANOVA results are displayed. Crab size treatments are as follows: UC-un-caged control, CC-caged control, Medium and Large crabs. 


\subsubsection{Attachment strength regressions}

The explanatory effect of stem and byssus thickness individually in simple regression models was low as $\mathrm{R}^{2}$ was $<0.394$. Hence, stepwise multiple regression models were used to test the correlation between the byssal parameters (stem thickness, byssus thickness and byssus number) and the attachment strength of individual mussels at Crail bay and Laverique bay. Several multiple regression models with high statistical significance were developed (Table 2.4). The regression slopes of Greenshell mussel attachment strength against the measured byssus characteristics also varied significantly (Fig 2.7), with byssus thickness at Crail Bay having a significantly higher regression slope than any of the other byssus characteristics. The regression slopes for stem thickness and byssus number were very similar for both sites (Fig 2.7).

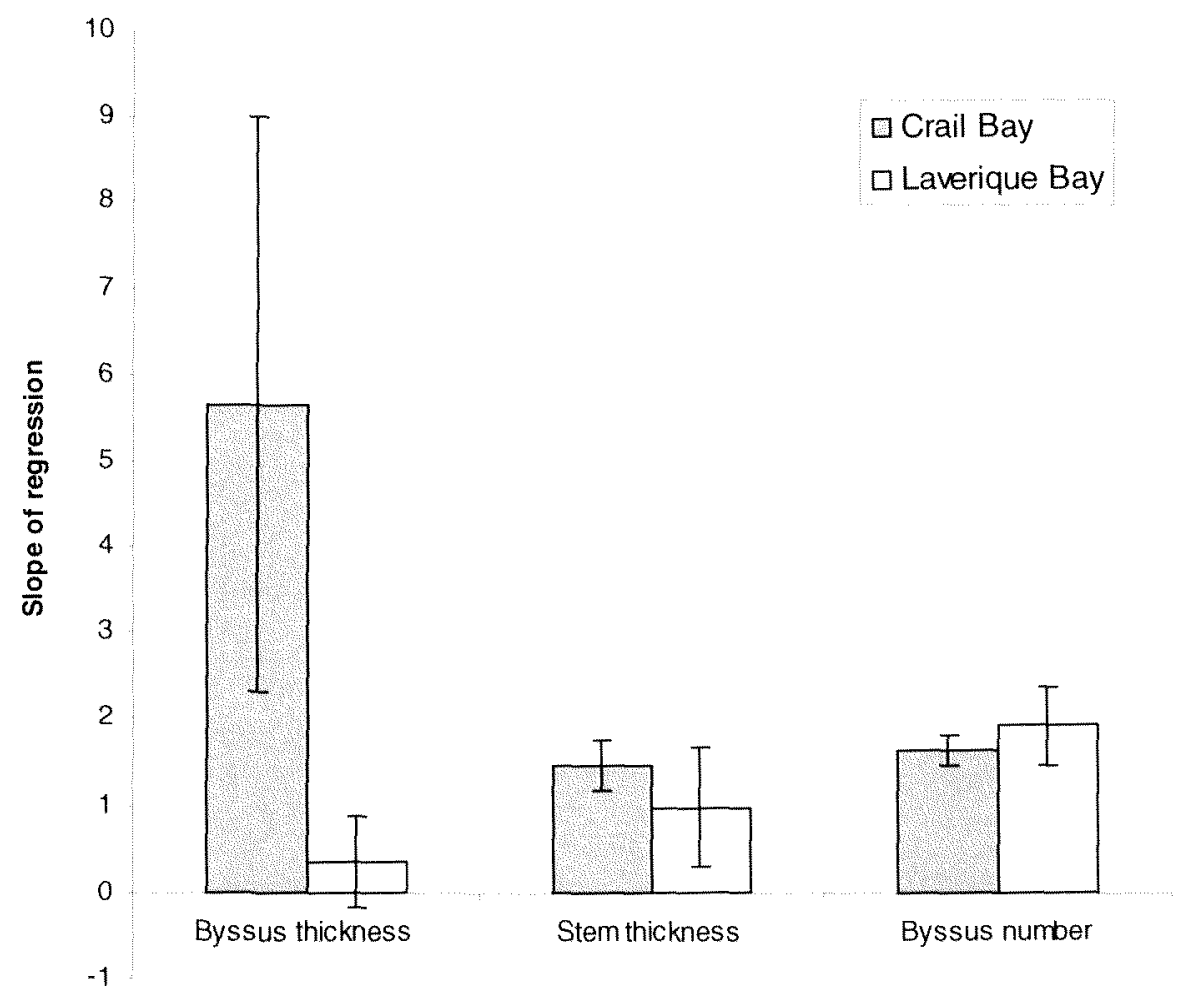

Figure 2.7: Slopes of regression of Greenshell mussel attachment strength against the three byssus characteristics included in the multiple linear regression analysis ( $\pm 1 \mathrm{SE}$ ) for each study site. 
Between the study sites there was a distinct difference in the number of byssus threads needed to achieve similar attachment strengths (Fig 2.8 a,b). For the same number of byssus threads the resulting attachment strength at Laverique Bay is at least twice as high as for the corresponding number of byssus threads at Crail Bay. At both study sites the spread of the data was large (Fig 2.8 a,b); at Laverique Bay byssus number was the only dependant variable included in the model however the correlation between byssus number and attachment strength was relatively low $\left(R^{2}: 0.123, p=>0.001\right)$.

Table 2.4: Multiple linear regression model summary describing the effects of the byssus charatcteristics byssus thickness, stem thickness and byssus number on the dependant variable mussel attachment strength. Byssus number, byssus thickness, stem thickness and attachment strength were $\log _{10}$ transformed to meet the assumptions of the analysis. Significant results are indicated in bold.

\begin{tabular}{|c|c|c|c|c|c|}
\hline \multirow{3}{*}{$\frac{\text { Site }}{\text { Crail Bay }}$} & & & Coefficients & Std. Error & $\mathrm{p}$-value \\
\hline & 1 & Constant & -0.387 & 0.299 & 0.197 \\
\hline & & $\begin{array}{l}\text { Byssus number } \\
\text { (Log10) }\end{array}$ & 1.640 & 0.174 & $<0.0001$ \\
\hline \multirow[t]{3}{*}{ Crail Bay } & 2 & Constant & -0.758 & 0.314 & 0.017 \\
\hline & & $\begin{array}{l}\text { Byssus number } \\
(\log 10)\end{array}$ & 1.686 & 0.170 & $<0.0001$ \\
\hline & & $\begin{array}{l}\text { Byssus thickness } \\
(\log 10)\end{array}$ & 7.924 & 2.568 & 0.002 \\
\hline \multirow[t]{2}{*}{ Laverique Bay } & 1 & Constant & -0.200 & 0.588 & 0.734 \\
\hline & & $\begin{array}{l}\text { Byssus number } \\
\text { (Log10) }\end{array}$ & 1.916 & 0.455 & $<0.0001$ \\
\hline
\end{tabular}

At Crail Bay two models describe the observed increase in attachment strength, byssus number alone describes $39.4 \%(p=>0.001)$ of the variation in attachment strength; however when byssus thickness is included in the model the descriptive value increases to $43.4 \%(\mathrm{p}=>0.001)$ 

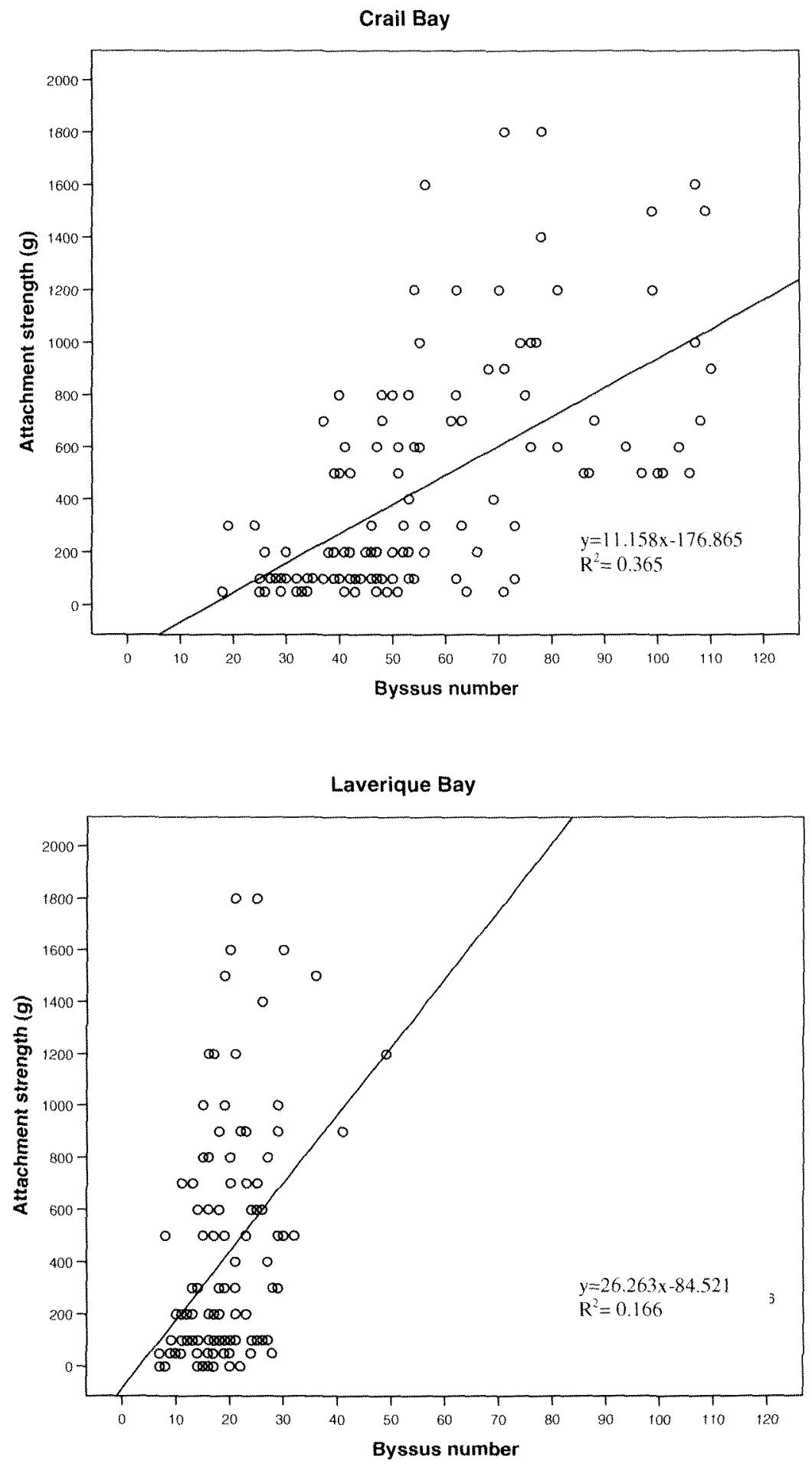

Figure 2.8: the effects of GreenshelliM mussel byssus number on the attachment strength of individual mussels at two study sites. Attachment strength is measured in grams of pressure required to dislodge mussels from the mussel spat ropes. $\mathrm{R}^{2}$ values and equations shown in figure. 


\subsection{DISCUSSION}

Abiotic factors such as wave action, temperature, salinity, seasonality and tidal regime have been widely documented to affect byssus production and byssus characteristics (Young 1985; Eyster and Pechenik 1987; Côte 1995; Hunt and Sheibling 2001; O'Connor et al. 2006). However despite the considerable commercial value of farmed GSMs in New Zealand, this study represents one of the first times a biotic factor, i.e. crab predation, has been directly implicated in the byssus production for cultivated GSM spat.

When $N$, minor were present on experimental mussel farm droppers in the Marlborough Sounds the GSM spat produced significantly more byssus threads. At Crail Bay, the summer trial site using small recently seeded spat, the thickness of the byssal root or stem also increased significantly in the presence of the decorator crabs (Fig.2.5 and 2.6). Bell and Gosline (1996) found that for three Mytilus species increased numbers of byssus threads directly resulted in increased attachment strength.

In the current study the changes in byssus production, and hence attachment strength of GSM spat, might be as a response to water-borne chemical cues emitted by the crabs. The release of chemicals such as pheromones and amino acids has been documented for various species of crabs (Ryan, 1966); the chemicals make it possible for potential prey species to detect the predators quickly (Côte, 1995). Many escape and predator avoidance behaviours have been linked to the chemical cues emitted by predators and chemicals secreted by conspecifics attacked by predators (Côte 1995, Reimer and Harms-Ringdahl 2001). For example Blue mussels (M. edulis) produce more, shorter and thicker byssus threads when held in water containing predatory crab effluent (Côte, 1995). By increasing byssus number when predators are present the 
mussels may be increasing their chances of survival. Attachment strength is inversely related to prey profitability and attractiveness; decapod predators need to remove a prey item from the substrate to be able to manipulate and consume it. Lin (1991) demonstrated this inverse relationship for ribbed mussels (Geukensia demissa) and the predatory blue crab (Callinectes sapidus); mussels tethered strongly to the substrate with nylon threads had a decreased risk of predation.

Chemical cues may not be the only explanation for the observed byssus changes in the experimental GSM spat.. Mussels produce significantly more byssus threads, and were consequently more thoroughly attached to the substrate, when they are agitated (Young 1985). Agitation has also been shown to affect byssus production in GSM spat; Alfaro (2006) demonstrated an increase in byssus number and attachment rate in juvenile GSM spat when air bubbles were added to experimental tanks, the air bubbles created mechanical stimuli that induced the observed byssal changes. It is reasonable to assume that the movement of crabs on the lines could cause enough agitation to have a similar effect on byssogenesis in GSM spat.

A number of previous studies have indicated that in Mytilus species attachment strength increases with increasing mussel size (Harger 1970, Witman and Gosline 1997 and Hunt and Scheibling 2001), However the results of the current study indicate that there was an inverse relationship between GSM sapt size and byssus number; the small mussels at Crail Bay produced more and thicker byssus threads when exposed to $N$. minor than the larger spat at Laverique Bay. The changes in byssus number and diameter were less pronounced and more irregular At Laverique bay. The Decorator crabs showed a preference for small mussels (see chapter 3, Fig 3.5) so small mussels could be more vulnerable to decorator crab predation, as mussel size increases the handling time 
increases and hence prey profitability decreases. For larger mussels the risk of predation by $N$. minor is lower, and the energetic costs of producing more and thicker byssus threads most likely outweighs the risk of predation. Byssus production in $M$. edulis depletes up to $44 \%$ of the carbon and $21 \%$ of the nitrogen budget allocated to summer growth (Hawkins, 1985). In the ribbed mussel (Aulacomya ater) byssus production accounts for around $15 \%$ of the total body energy and there is a direct trade-off between byssus production and growth (Griffiths and King 1979). The energetic costs in $P$. canaliculus have not been documented but it is realistic to assume some trade-off will be made to produce more and thicker byssus threads.

Although the two study sites had similar physical characteristics, the seasonality of the two trials could also be contributing to the observed differences in byssus characteristics and attachment strength. The average temperature at Crail Bay during the experiment would have been approximately $4^{\circ} \mathrm{C}$ lower than during the Laverique Bay trial (table 2.2). Byssus production in $M$. edulis has been shown to increase when temperature increases (Allen et al. 1976, Young 1985), this increase has been attributed to changes in the mussels' metabolic rate and increased mobility of the mussels at higher temperatures (Allen et al. 1976). The GSMs in this study showed an inverse relationship between temperature and byssus production; byssus number was higher at the winter study site. Byssus threads are produced in response to a number of contributing factors (Young 1985), in this study the effects of agitation due to increased wind and higher frequency of storms in the winter months may have over-ridden the influence of temperature on byssus production.

Previous studies regarding predator induced byssogenisis and changes in byssus thickness were performed under controlled conditions in laboratory settings. This study 
was entirely field based which can have positive and negative consequences when trying to draw conclusions based on the results obtained. Although it may be more appropriate to draw conclusions regarding the focal ecosystem from results of trials conducted within that particular ecosystem; the environmental variables discussed above need to be taken into account when drawing conclusions regarding the extent to which $N$. minor presence affects byssus production. Randomization of treatments across the section of backbone selected should have mediated some of these potential environmental biases.

This study demonstrated that the presence of a potential predator can influence byssus production in cultivated GSM spat. This could have economic implications for the mussel industry by increasing spat retention which is regularly less than $5 \%$. Spat losses can cost commercial GSM farmers hundreds of thousands of dollars annually, due to farmers having to purchase more spat or spend more time and effort catching spat to compensate for these (Sim-Smith 2006). This is a highly inefficient method of farming and an increase in spat retention as small as $1 \%$ could have significant economic benefits. Spat attachment strength also has implications in spat survival particularly during the stripping of spat for reseeding. The field trials in this study were restricted in site and replication by industry allocations of GSM spat. Temporal replication within a site using spat from the same source would add weight to the results and conclusions drawn from the results. Further research incorporating the aforementioned suggestions is needed to improve the generality and applicability of the results. 


\section{Chapter 3: Predation by Notomithrax minor on Greenshell mussel spat: spat retention, prey size selection and predation rates}

\subsection{INTRODUCTION}

Crabs are common predators in many marine ecosystems and due to their behaviour and feeding they can affect prey population densities and the abundance of many non-target co-existing organisms (Woods, 1993). Not only can crabs directly reduce prey numbers in a given area via predation, the long term indirect effects of predator behaviour may disrupt the fitness of prey individuals and subsequently the persistence of the prey populations (Leonard et al. 1999; Mistri, 2004).

Brachyuran crabs prey heavily on marine bivalves, including many mussel species (Mistri 2004). Due to their often high abundance and cosmopolitan distribution, it is important to understand the prey preferences of these crabs and how they influence the behaviour and morphology of their prey species. Crab foraging and predatory behaviour can have particularly high costs, both economically and ecologically, when the crabs or their prey are of commercial importance (Richards et al. 1999, SanchezSalazar et al. 1987, Woods, 1993).

In this study the focal prey species, the endemic New Zealand (NZ) Greenshell ${ }^{\mathrm{TM}}$ mussel Perna canaliculus (hereafter referred to as GSM), is the most extensively farmed marine bivalve species in New Zealand (Carton et al., 2007) producing export market revenue of more than NZ\$180 M (NZ Mussel Industry Council Ltd Database 2006). The focal predator species, the camouflage crab Notomithrax minor, has no direct commercial value. 
The GSM aquaculture industry is largely dependant on wild spat caught on either local spat-rope collectors or macroalgae washed up on Ninety Mile Beach (Buchanan, 1994; Carton et al. 2007; Jeffs et al. 1999). Wild spat settlement is notoriously unreliable and once the spat have been collected the retention rates are extremely variable, with often only about $2 \%$ of all spat applied to grow-out lines being retained to grow to a harvestable size (Hayden 1994, Webb and Heasman 2006). The inefficient use of spat and poor spat retention rates ultimately result in large production and financial losses (Carton et al. 2007), costing the aquaculture industry hundreds of thousands of dollars annually (Sim-Smith 2006).

Possible causes of the low spat retention rates include: predation (Sim-Smith 2006), competition (Hickman 1979, Hayden 1984) and environmental conditions (Alfaro 2005, Sim-Smith 2006). Juvenile GSMs (spat) are particularly susceptible to predation; the small mussels have thin shells, weak byssal attachment and are farmed in high densities which in itself attract many predator species. Anecdotal evidence from NIWA and Sealord Group Ltd. suggests that predation is one of the principal factors leading to substantial decreases in spat retention. The current study investigates the effects of a common invertebrate predator, the decorator crab $N$. minor, on GSM spat. This chapter incorporates field caging experiments to assess predation rates on locally caught $P$. canaliculus and M. galloprovincialis spat and Kaitaia sourced GSM spat in Pelorus Sound, Marlborough Sounds, NZ. Crab prey size preferences and satiation levels were also investigated in a laboratory setting using wild caught $N$. minor and hatchery raised $P$. canaliculus. The specific aim of this work is to better understand the effect that $N$. minor has on GSM spat retention rates, and indirectly what sort of financial impact this has on the NZ GSM industry. 
This focus of this chapter us to test the following null hypotheses: (1) decorator crabs ( $N$. minor) have no effect on the survival and growth of GSM spat in the Marlborough Sounds, (2) decorator crabs show no preference for a particular size of GSM spat, and (3) decorator crabs have the ability to consume an unlimited number of GSM spat.

\subsection{METHODS}

\subsection{Field trial study site}

This research was conducted in Pelorus Sound (in the Marlborough Sounds), which stretches $35 \mathrm{~km}$ from the Pelorus River at Havelock in the south (inner Sounds), to the Cook Strait in the north (outer Sounds). Pelorus Sound has several major arms including the Tawhitinui Reach and Kenepuru Sound (Fig.3.1).

Data relating to the environmental conditions of Pelorus Sound, including salinity, Chl $a$ concentration, nutrient and particulate fluxes, were taken from Hickman et al. (1991) and Gibbs et al. (1992).

\subsubsection{Laverique Bay and Crail Bay}

During the two study periods, winter 2006 (June-September) and summer 2006/2007 (November-January), caging experiments were carried out on GSM spat farms in Pelorus Sound, the Marlborough Sounds, NZ. The farms were located at two discrete sites within Pelorus Sound (Crail Bay and Laverique Bay). The sites are spat catching and grow-out sites, selected for their water quality, phytoplankton concentrations and relatively low density of predatory fish. Both sites are located in Beatrix Bay, in eastern Pelorus Sound. 
Table 3.1: Site specific temperature, salinity and chlorophyll a measurements for Laverique Bay and Crail Bay. The data is from 2005-2006, for the summer and winter periods during which current testing took place.

\begin{tabular}{|c|c|c|c|c|c|c|}
\hline \multirow[t]{2}{*}{ WEEK } & \multicolumn{2}{|c|}{ TEMPERATURE $\left({ }^{\circ} \mathrm{C}\right)$} & \multicolumn{2}{|c|}{ SALINITY (PPM) } & \multicolumn{2}{|c|}{ CHL A ( $\mu$ G. LITRE ${ }^{-1}$ ) } \\
\hline & $\begin{array}{c}\text { LAVERIQUE } \\
\text { BAY }\end{array}$ & CRAL BAY & $\begin{array}{c}\text { LAVERIQUE } \\
\text { BAY }\end{array}$ & CRAIL BAY & $\begin{array}{c}\text { LAVERIQUE } \\
\text { BAY }\end{array}$ & CRAIL BAY \\
\hline DEC 04-DEC10 & 16 & 17.5 & 33.5 & 33,3 & $0.1-1.2$ & $0.1-1.3$ \\
\hline DEC 18-DEC 25 & 17.5 & 18 & 34 & 34 & $0.1-0.3$ & $0.1-1.5$ \\
\hline JUN 12-JUN 18 & 12.5 & 12.7 & 33.2 & 33.8 & $0.8-1.2$ & $0.9-1.2$ \\
\hline JUN 26-JUL 02 & 11.7 & 12.0 & 33.6 & 33.9 & $1.0-1.7$ & $0.8-1.2$ \\
\hline
\end{tabular}

Data adapted from NIWA 'Marlborough Sounds Environmental Monitoring Programme' (2005-2006).

Both study sites are situated in a large eastern branch of the Pelorus Sound. The branch contains three major bays: Beatrix, Crail and Clova Bay, all of which support a large number of marine farms (Fig. 3.1). Crail Bay is on the north western shore of Beatrix Bay $\left(41^{\circ} 05^{\prime} 393 \mathrm{~S}, 173^{\circ} 58^{\prime} 205 \mathrm{E}\right)$ and Laverique Bay $\left(41^{\circ} 01^{\prime} \mathrm{S}, 174^{\circ} 02^{\prime} \mathrm{E}\right)$ is in the south eastern area of Beatrix Bay(Fig. 3.1). The Crail Bay site is situated in relatively deep water (approx $30 \mathrm{~m}$ ), whereas the Laverique Bay site is around $25 \mathrm{~m}$ deep. Both sites are relatively exposed to wind and water movement, they also have similar salinity, temperature and Chl $a$ characteristics (Table 3.1).

\subsubsection{Experimental design}

Cages were placed on droppers at both sites to retain experimental crabs within a specific area of spat line. The cages used were commercial shellfish grow-out apparatus called Aquapurses. The specific Aquapurses used were $0.8 \mathrm{~m}$ long with $8 \mathrm{~mm}$ mesh; a $20 \times 20 \mathrm{~mm}$ hole was cut in the top and bottom of each cage to allow the cage to be attached around the mussel line. Aquapurses are manufactured by Tooltech Pty Ltd (Australia) and are used commercially in oyster culture in NZ.

Long-lines in the interior of the farms were selected to minimize the variation of exposure among experimental droppers. On the target long-line, top and bottom 
locations were avoided: cages were placed at $4 \mathrm{~m}$ depth on randomly selected $8 \mathrm{~m}$ long single droppers, one cage per dropper. At Laverique Bay, the farm is set-up using single droppers which consist of $8 \mathrm{~m}$ lengths of commercial spat catching rope, commonly known as Christmas tree rope. Cages were placed on the northwest side of the backbone at $4 \mathrm{~m}$ depth, but on one dropper the cage was placed shallower $(3 \mathrm{~m})$, because of scuffing during earlier movement of the spat lines which had removed most of the mussels at the desired depth.

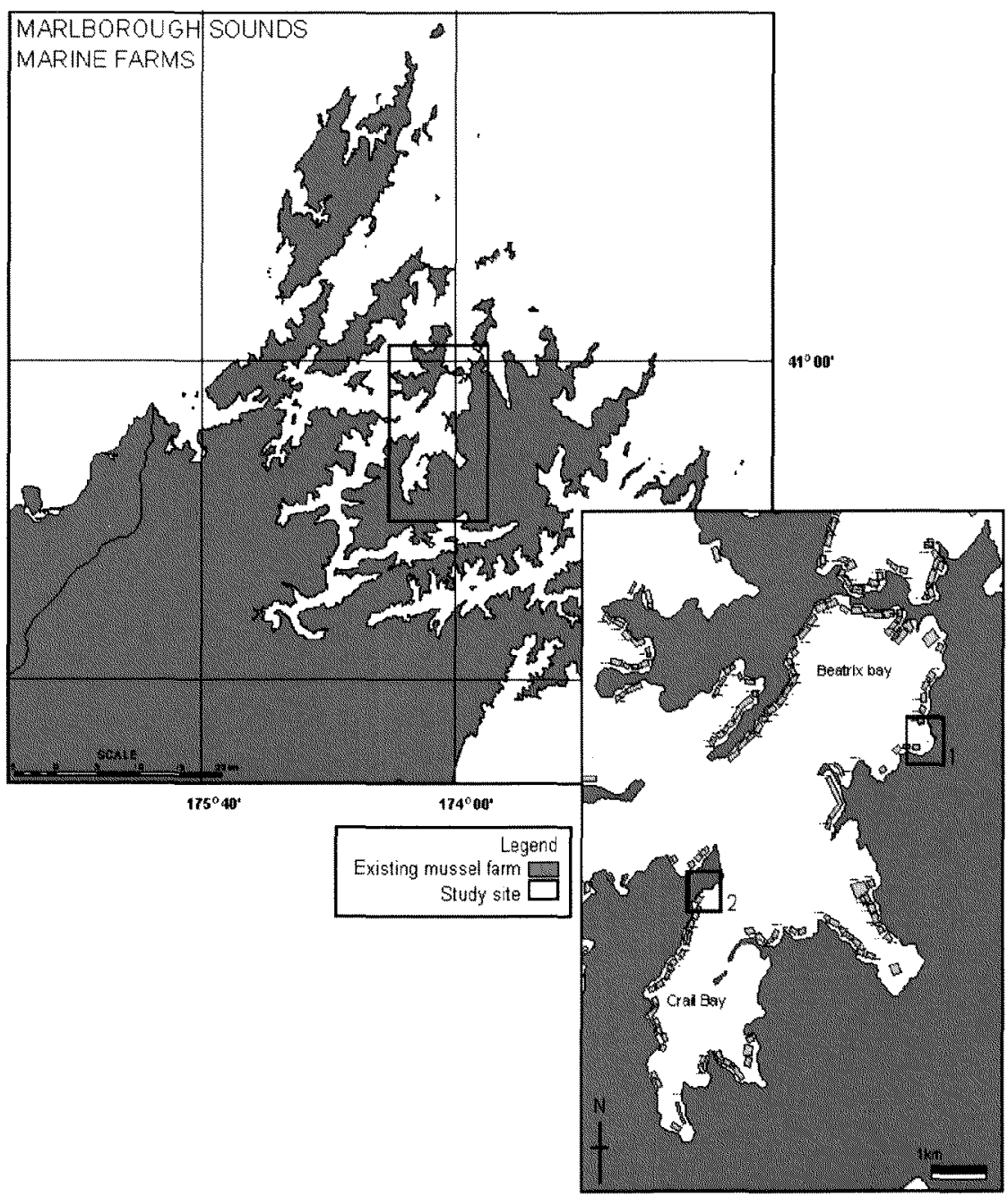

Figure 3.1: The location of the study sites. Boxes indicate site locations within Pelorus Sound, Laverique Bay to the north (1) and Crail Bay to the south (2). Locations of existing mussel farms are from Marlborough District Council data (2003). 
At Crail Bay, continuous $12 \mathrm{~m}$ long droppers were used and single cages were placed on droppers on the east side of the backbone (Fig. 3.2).

The droppers at Laverique Bay were placed in the water on the $8^{\text {th }}$ of March 2006 to allow mussel spat to settle naturally on the lines. The field trial commenced on the $25^{\text {th }}$ of July 2006 and by this time the spat had an average total shell length (TSL measured across the anterior dorsal-posterior ventral axis) of $16.8 \mathrm{~mm} \pm 0.79(95 \%$ C.I.). GSM density was considerably higher in the uppermost $1 \mathrm{~m}$ of the droppers. Upon visual inspection at commencement of the field trial there was no substantial 'oversettlement' of $M$. galloprovincialis, although numerous other species were also present on the lines, including various scallop species, bryozoans, algae and a small number of decorator crabs between 10-20 mm total carapace width (TCW measured at the widest point of carapace).

The GSMs at Crail Bay were sourced from Kaitaia spat collected at Ninety Mile Beach and seeded mechanically onto the spat ropes $3 \mathrm{wk}$ before commencement of the field trial $\left(23^{\text {rd }}\right.$ November 2006). Due to the spat being seeded onto the line there was minimal over-settlement of other species; $M$. galloprovincialis were present in very low numbers. At the start of the field trial at Crail Bay the average size of the GSM spat was $4.86 \mathrm{~mm} \pm 0.56$ (95\% C.I.). Some cotton stocking was still visible but the mussels were readily accessible to predators.

Crabs were collected 10 days before commencement of the field trials and held in large ( $1.3 \mathrm{~m}$ width $\times 1.3 \mathrm{~m}$ height $\times 0.75 \mathrm{~m}$ depth) black plastic tanks in the laboratory at the Cawthron Aquaculture Facility, Nelson. Crabs were fed to excess $24 \mathrm{hr}$ after collection with fresh GSM flesh. $24 \mathrm{hr}$ before onset of the field trial the crabs were sorted into the relevant treatments (detailed below) and weighed to $0.1 \mathrm{~g}$, to permit 
estimation of crab growth over the experimental period. Crabs were transported to each site in a $90 \mathrm{~L}$ bin filled with fresh seawater with two battery powered air pumps oxygenating the water. The caged section of mussel rope was checked for any local crabs, which were removed before the experimental crabs were introduced.

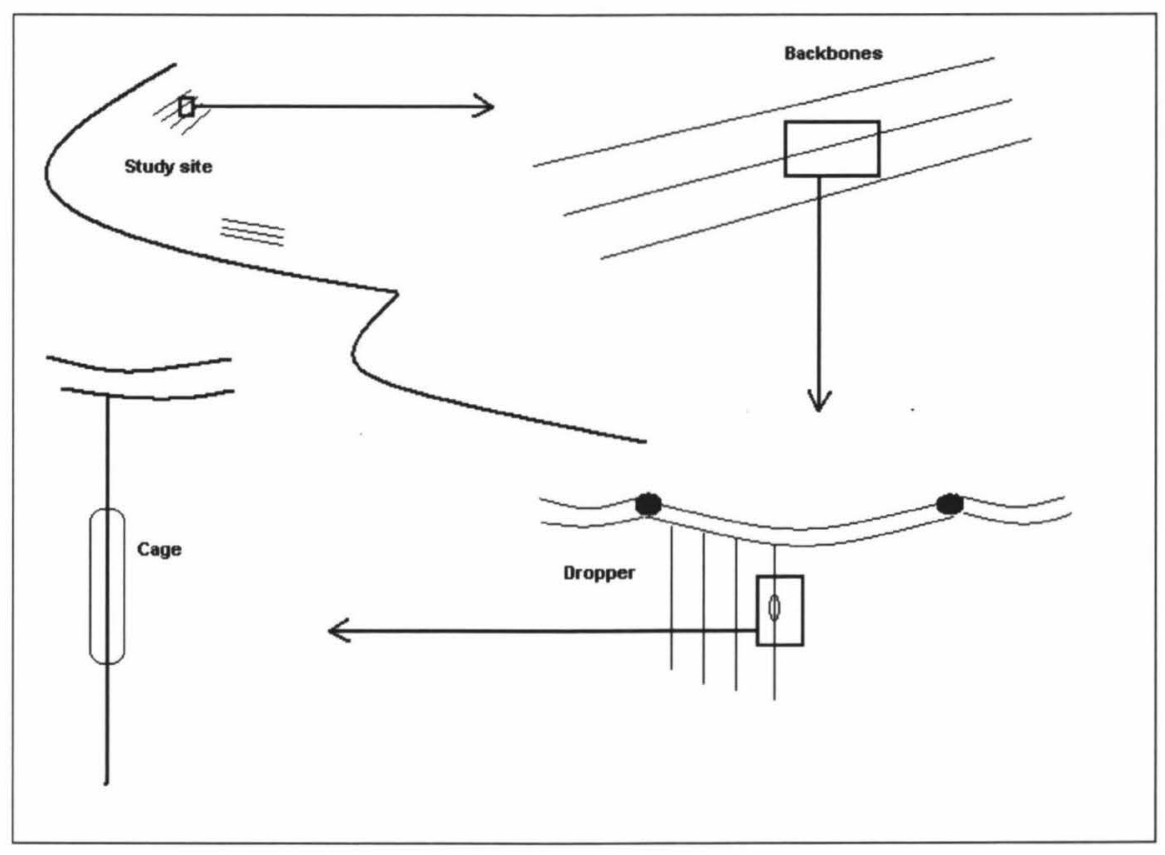

Figure 3.2: schematic illustration of the construction of a GSM mussel farm in the Marlborough Sounds, outlining terms in the methodology (study site, backbone, dropper and cage) and the positioning of the experimental cages.

The effects of crab density and size on GSM growth, mortality and attachment strength, different crab treatments were tested. Treatments included: high $(\mathrm{H})$ (12 crabs) and low density (L) (3 crabs), of both large (La) and medium sized crabs (M). Large males had TCW $>20 \mathrm{~mm}$, large females were $>15 \mathrm{~mm}$, medium males were $15-20 \mathrm{~mm}$, and medium females were 10-15 mm TCW. A zero density caged treatment (CC) and an uncaged control (UC) were also included in the trails to assess caging effects. Only medium and large crabs were used because of the cryptic nature of these crabs and the logistic difficulty involved with their collection. At Laverique Bay, 3 replicates of each 
treatment were retrieved after $6 \mathrm{wk}$ and again after $11 \mathrm{wk}$, At Crail Bay, 8 replicates of each treatment were retrieved after $8 \mathrm{wk}$ (Table 3.2). The differences in methodology for the two study sites was due to the fact commercial GSM spat were used, thus experimental design was limited by the quantity and type of spat made available by commercial GSM farmers. Optimally the entire Laverique Bay trial would have been run for 11 weeks due to it being run in the winter. Predation rates and GSM growth rates are generally lower during the winter months. However the GSM spat at Laverique Bay needed to start to be harvested for on growing after 6 weeks, only half of the spat was made available for longer term experimentation (11 wk). The Crail Bay trial was run for a shorter period due to the fact it was run in the summer months and no limitations were placed on experimental design by the GSM farmers at this study site.

\subsubsection{Line retrieval}

At each retrieval date (Table 3.2) the spat lines were lifted and the $0.8 \mathrm{~m}$ section inside the cage was removed. On un-caged control lines a $0.8 \mathrm{~m}$ section of line was cut at $4 \mathrm{~m}$ depth. The top and bottom $10 \mathrm{~cm}$ of the caged and un-caged samples were excluded due to clumping of mussels against the ends of the cages. The $60 \mathrm{~cm}$ sections of line were soaked in a $10 \%$ chlorine solution for $24 \mathrm{hr}$, thoroughly scrubbed and inspected to remove any GSM spat and other bivalves. The spat and other bivalves were then sieved through a $2 \mathrm{~mm}$ sieve and rinsed to remove any residual fouling. The TSL (total shell length) of a haphazardly selected sub-sample of 25 GSM spat was recorded for each sample. The total number of GSM spat for each sample was recorded. 
Table 3.2: Detailed outline of experimental design at Laverique Bay and Crail Bay

\begin{tabular}{|c|c|c|c|c|c|}
\hline \multirow[t]{2}{*}{ Treatment } & \multirow[t]{2}{*}{ Crab Size } & \multicolumn{2}{|c|}{ Crail Bay } & \multicolumn{2}{|c|}{ Laverique Bay } \\
\hline & & Put out & Retrieved & Put out & Retrieved \\
\hline \multirow[b]{2}{*}{ High $(H)$} & Medium (M) & $\begin{array}{l}11 / 07 / 06 \\
11 / 07 / 06\end{array}$ & $\begin{array}{l}22 / 08 / 06 \text { (6wk) } \\
22 / 10 / 06 \text { (11wk) }\end{array}$ & $23 / 11 / 06$ & $18 / 01 / 07$ (8wk) \\
\hline & Large (La) & $\begin{array}{l}11 / 07 / 06 \\
11 / 07 / 06\end{array}$ & $\begin{array}{l}22 / 08 / 06 \text { (6wk) } \\
22 / 10 / 06(11 w k)\end{array}$ & $23 / 11 / 06$ & $18 / 01 / 07$ (8wk) \\
\hline \multirow[b]{2}{*}{ Low (L) } & $\operatorname{Medium}(M)$ & $\begin{array}{l}11 / 07 / 06 \\
11 / 07 / 06\end{array}$ & $\begin{array}{l}22 / 08 / 06 \text { (6wk) } \\
22 / 10 / 06(11 w k)\end{array}$ & $23 / 11 / 06$ & $18 / 01 / 07$ (8wk) \\
\hline & Large(La) & $\begin{array}{l}11 / 07 / 06 \\
11 / 07 / 06\end{array}$ & $\begin{array}{l}22 / 08 / 06 \text { (6wk) } \\
22 / 10 / 06 \text { (11wk) }\end{array}$ & $23 / 11 / 06$ & $18 / 01 / 07$ (8wk) \\
\hline Zero & Caged $(C C)$ & $\begin{array}{l}11 / 07 / 06 \\
11 / 07 / 06\end{array}$ & $\begin{array}{l}22 / 08 / 06 \text { (6wk) } \\
22 / 10 / 06 \text { (11wk) }\end{array}$ & $23 / 11 / 06$ & $18 / 01 / 07$ (8wk) \\
\hline Uncaged & Control (UC) & $\begin{array}{l}11 / 07 / 06 \\
11 / 07 / 06\end{array}$ & $\begin{array}{l}22 / 08 / 06 \text { (6 wk) } \\
22 / 10 / 06 \text { (11wk) }\end{array}$ & $23 / 11 / 06$ & $18 / 01 / 07$ (8wk) \\
\hline
\end{tabular}

\subsubsection{Laboratory trials}

The $N$. minor used in the laboratory experiments were collected by hand from mussel farms in Pelorus Sound, 10 days prior to trial commencement. The mussels used were a combination of hatchery-raised and wild spat obtained from spat catching farms in Pelorus Sound and the Cawthron Aquaculture facility. The animals were maintained in flow-through indoor tanks at the Cawthron Aquaculture facility. Only undamaged crabs were used in experiments. The carapace width $(\mathrm{CW})$ of each of the test crabs and the total shell length (TSL) of the test mussels was determined using calipers. TSL was measured across the anterior dorsal-posterior ventral axis. The sex of the crab was also noted. The test crabs were starved for $48 \mathrm{hr}$ prior to experimentation to standardize hunger levels and each crab was used only once.

Experiments were conducted in 91 plastic aquaria supplied with filtered, temperature controlled seawater $\left(17-18^{\circ} \mathrm{C}\right.$, salinity $34-35 \mathrm{ppt}$, water turnover rate: 5 
min). Aquaria were also supplied with individual air lines. Individual test crabs were placed haphazardly in one of 26 replicate aquaria with bivalve prey. Experiments were conducted with a $12 \mathrm{~h}$ photoperiod. The test aquaria were covered in $1 \mathrm{~mm}$ mesh to ensure no mussels or crabs escaped. At the end of each experiment, the crab was removed, and the live and dead mussels, along with shell fragments, were recovered. Only mussels with visible signs of crab predation (chipped or crushed shells) were included in predation estimates. Chipped shells that were not opened by the crab and dead bivalves with intact shells (non-predatory mortality) were not included in predation estimates. The difference in the number of prey at the start of the trial and end of the trial represented the number of prey consumed.

\subsubsection{Prey size selection}

The objective of this experiment was to determine predator size preferences of prey eaten by 'medium' sized male and female $N$. minor (males, 20-35 mm TCW; females, $15-27 \mathrm{~mm}$ TCW). This size range is representative of the majority of the crabs collected on GSM farms in Pelorus Sound. A standard array of various sized mussel spat ( $<5$ mm, 30 mussels; 5-10 mm, 15 mussels; 10-15 mm, 7 mussels; $>15$ mm, 3 mussels) were added to individual small plastic mesh bags ( 80 x $80 \mathrm{~mm}, 1 \mathrm{~mm}$ mesh). High numbers of small mussels and low numbers of large mussels were used in this experiment to balance out encounter rate and biomass of each size class. These bags were held in the corresponding test aquaria $72 \mathrm{hr}$ before initiation of the trials to allow byssal attachments to form and the mussels to clump naturally. At the start of each experiment, the bags were opened up, laid flat and attached to the base of each aquarium to make all enclosed mussels accessible to the crabs. After 3 days the number of mussel 
spat remaining in each size class was determined. In order to eliminate the possibility of secondary settlement and migration of the mussel spat, three control treatments (no crabs) were included in the experiment.

\subsubsection{Satiation level}

The purpose of this experiment was to determine the number of small GSM spat that individual and multiple 'medium' sized male and female $N$. minor (males, 20-35 mm TCW; females, $15-27 \mathrm{~mm}$ TCW) could consume per hour. The 16 test aquaria contained 0 ( $1 \mathrm{x}$ control) or $1(15 \mathrm{x}$ treatment $)$, crab selected at random for sex and size within the 'medium' size range (males, 20-35 mm TCW; females, 15-27 mm TCW). At the start of the experiment, 100 small $P$. canaliculus $\leq 5 \mathrm{~mm}$, were added to each of the experimental aquaria. The mussels had been left to attach to small $(40 \mathrm{x} 50 \mathrm{~mm})$ hessian wrapped plastic panels for 24 hours pre-experimentation allow for byssal attachment.

After $1 \mathrm{hr}$ the initial mussel panel in each aquarium was removed and a new identical panel was introduced. The number of mussels eaten was then recorded; this process was repeated every hour from 0900 to 1700 daily for three consecutive days.

\subsubsection{Statistical analysis}

The statistical and data packages SPSS 14.0 for Windows (SPSS Incorporated, Chicago, Illinois) and SigmaPlot 9.01 for Windows (SyStat Software, Inc.) were used for graphing and analysis of the data.

\subsubsection{Field trials}

Box-plots for GSM number and GSM spat size were constructed to assess normality and to help recognize any outliers. Any outliers were checked against the raw data and if they were genuine outliers, the data points were deleted. Cochran's Q test 
was used to assess the equality of variances across the crab density, size and control treatments. GSM mussel number data were square root transformed for Crail Bay and $\log _{10}$ transformed for Laverique Bay to meet the requirements of parametric Analysis of Variance (ANOVA). GSM size data were $\log _{10}$ transformed for both sites to also meet the requirements of parametric ANOVA. The data for mussel number and size were standardized for varying trial temporal durations by dividing the values by the duration (days) of each trial.

The site-specific standardized data were subjected to a two-way ANOVA to test the null hypothesis $\left(H_{o}\right)$ that crab $(N$. minor $)$ density and size had no effect on the number of GSM spat and size of GSM spat found on mussel culture droppers in Pelorus Sound. A two-way ANOVA test was run for each of the two response variables (mussel number and size). Separate ANOVA analyses were chosen over MANOVA tests due to statistically significant correlations between the response variables (Pearson and Kendall's tau-b tests in SPSS). In the ANOVA model, crab density and crab size were considered to be fixed factors. Results significant $\alpha=0.05$ were subject to post hoc Tukey tests (pair-wise comparisons) to establish the location of significant differences

\subsubsection{Laboratory trials}

For the prey size selection data, the percent of mussels eaten in each mussel size class (small $(<5 \mathrm{~mm})$, small-medium $(5-10 \mathrm{~mm})$, medium-large $(10-15 \mathrm{~mm})$, large $(>15$ $\mathrm{mm})$ ) was calculated for each of the crab experimental (male, female and control) categories. Percent values were used because a different number of mussels were used in each of the 4 mussel size classes. 
Cochran's Q test was used to assess the equality of variances across the crab sex and control treatments, and Normal $Q-Q$ plots were constructed to test the normality of the data. The data did not meet the normality requirements of parametric ANOVA, even after a number of data transformations including $\log _{10}$ and square root. Therefore, the non-parametric Kruskal-Wallis test was used to test the null hypothesis $\left(H_{o}\right)$ there was no relationship between mussel size and the proportion of mussels consumed by male and female $N$. minor. For the Kruskal-Wallis analysis the data set was split into male and female crabs and ranked. Mussel size classes were used as the grouping (independent) variable and the percent of mussels eaten per size class was used as the dependent variable.

The average numbers of mussels eaten per hour and per day were calculated from the data collected in the satiation trial. The mean number of mussels eaten per day was calculated for male and female crabs, and a control treatment. Normal $Q-Q$ plots were constructed to assess the normality of the data and Cochran's Q test was used to assess the equality of variances across the three treatment days. The number of mussels eaten per day was $\log _{10}$ transformed to meet the requirements of parametric ANOVA. The data were subject to two-way ANOVA to test the null hypothesis $\left(H_{o}\right)$ that the number of GSM spat consumed by male and female $N$. minor in independent of both crab sex and time (day). In the ANOVA model, day and crab sex were considered to be fixed factors. The locations of significant differences $(\alpha=0.05)$ were identified using post hoc Tukey tests (pair-wise comparisons). 


\subsection{RESULTS}

\subsubsection{Field trials}

Greenshell (Perna canaliculus) mussel density showed different responses to decorator crab (Notomithrax minor) crab density and size at the two study sites. At Laverique Bay, the initial densities $\left(\mathrm{m}^{-1}\right)$ of GSM spat was lower than at Crail Bay $(\mathrm{LB}=$ $\left.989.2 \mathrm{~m}^{-1} ; \mathrm{CB}=4638.0 \mathrm{~m}^{-1}\right)$. Crab density and crab size did not significantly affect GSM density at Laverique Bay (Table 3.3).

At Crail Bay, crab density had a marginally significant effect on GSM density, whereas crab size did not significantly effect on the number of GSMs per meter of spat dropper (Fig 3.3, Table 3.3). The two crab treatments had significantly lower GSM densities than the two control treatments (Fig 3.3, Table 3.3), but were not significantly different from each other (post hoc Tukey test, $\mathrm{p}<0.05$ )

\section{Crail Bay}

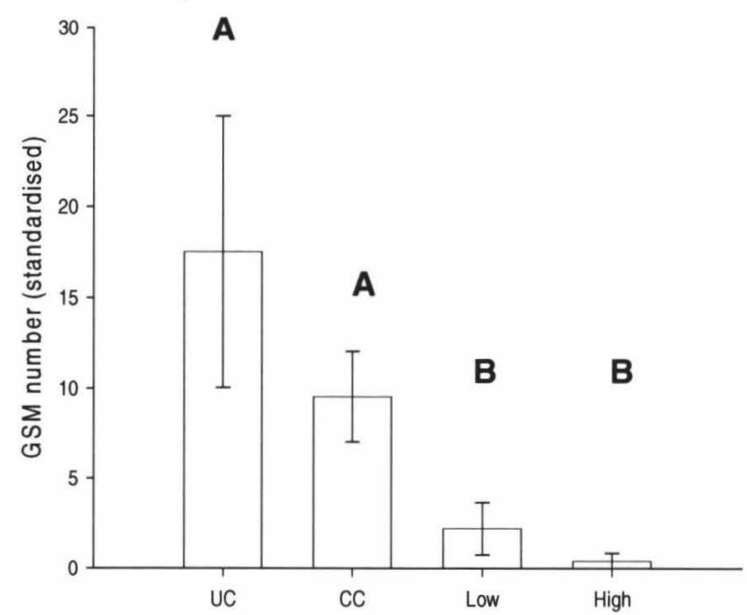

Laverique Bay

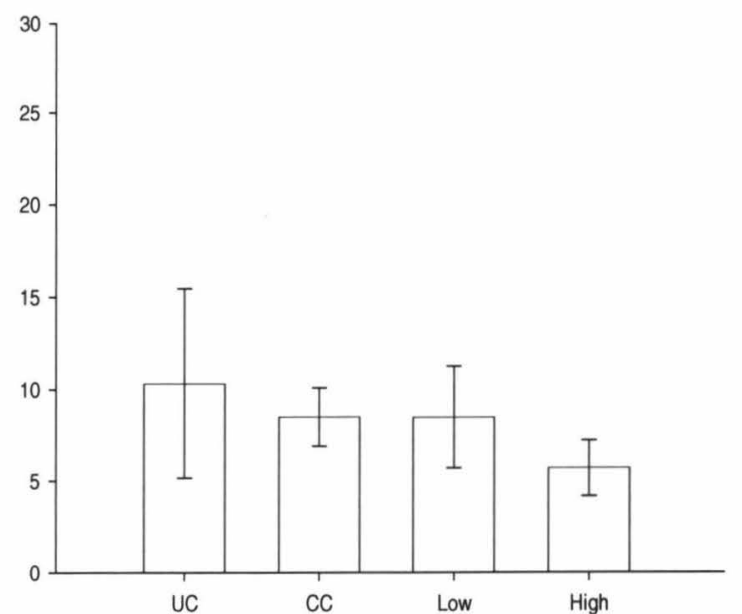

Fig 3.3: Effects of potential predator (N. minor) presence on P. canaliculus (Greenshell mussel) density (mussels $\mathrm{m}^{-2}$ ) on spat catching ropes at the two study sites, Crail Bay and Laverique Bay. Mussel number was standardised for days by dividing the mussel number by the number of days the experiment ran for ( 42 and 77 days at Laverique Bay, 56 days for Crail Bay). The differences in $P$. canaliculus densities were statistically significant at Crail Bay but not at Laverique Bay. Letters represent statistically significant post hoc groupings calculated using Tukey tests following ANOVA. Crab density treatments are as follows: UC - un-caged control, CC - caged control, Average - 3 crabs per cage, High - 12 crabs per cage. 
At Laverique Bay average GSM TSL (total shell length) was higher than at

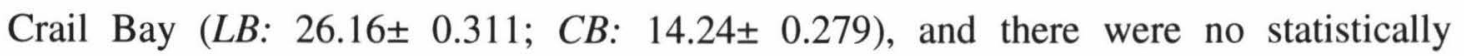
significant differences in GSM TSL across the experimental treatments (Fig 3.4, Table

Table 3.3: ANOVA table for GSM ( $P$. canaliculus) density, and GSM size for both study sites Laverique Bay (LB) and Crail Bay (CB). Crab density and Crab size were considered to be fixed factors in the analysis. For Crail Bay, GSM numbers were square root transformed; at Laverique Bay these dependant factors were $\log _{10}$ transformed to meet the assumptions of parametric ANOVA. GSM size was $\log _{10}$ transformed for data from both Laverique Bay and Crail Bay. Significant results are indicated in bold. $F$-ratios calculated from Type III SS

\begin{tabular}{|c|c|c|c|c|c|c|c|c|c|}
\hline & \multirow[t]{2}{*}{ FACTOR } & \multicolumn{2}{|c|}{ DF. } & \multicolumn{2}{|r|}{ MS } & \multicolumn{2}{|c|}{$\mathbf{F}$} & \multicolumn{2}{|c|}{ P-VAlue } \\
\hline & & LB & CB & LB & $\mathrm{CB}$ & LB & $\mathrm{CB}$ & LB & $\mathrm{CB}$ \\
\hline \multirow{3}{*}{$\begin{array}{c}\text { GSM } \\
\text { NUMBER }\end{array}$} & CRAB DENSITY & 1 & 1 & 0.162 & 2.535 & 3.322 & 4.227 & 0.077 & 0.049 \\
\hline & CRAB SIZE & 1 & 1 & 0.005 & 0.604 & 0.095 & 1.007 & 0.760 & 0.324 \\
\hline & $\begin{array}{c}\text { CRAB } \\
\text { SIZE*DENSITY }\end{array}$ & 1 & 1 & 0.017 & 0.014 & 0.353 & 0.024 & 0.556 & 0.878 \\
\hline \multirow{3}{*}{$\begin{array}{l}\text { GSM } \\
\text { SIZE }\end{array}$} & CRAB DENSITY & 1 & 1 & 0.221 & $<0.001$ & 2.656 & 0.068 & 0.112 & 0.769 \\
\hline & CRAB SIZE & 1 & 1 & 0.276 & 0.028 & 3.317 & 6.163 & 0.077 & 0.019 \\
\hline & $\begin{array}{c}\text { CRAB } \\
\text { SIZE*DENSITY }\end{array}$ & 1 & 1 & 0.062 & 0.002 & 0.750 & 0.499 & 0.392 & 0.486 \\
\hline
\end{tabular}

Crab density did not have a significant effect on GSM TSL at Crail Bay (Table 3.3, Fig 3.4), although GSMs in caged control and medium crab size treatments had significantly higher TSLs than un-caged control and large crab size treatments (post hoc Tukey test $\mathrm{p}<0.05)$. 
Crail Bay

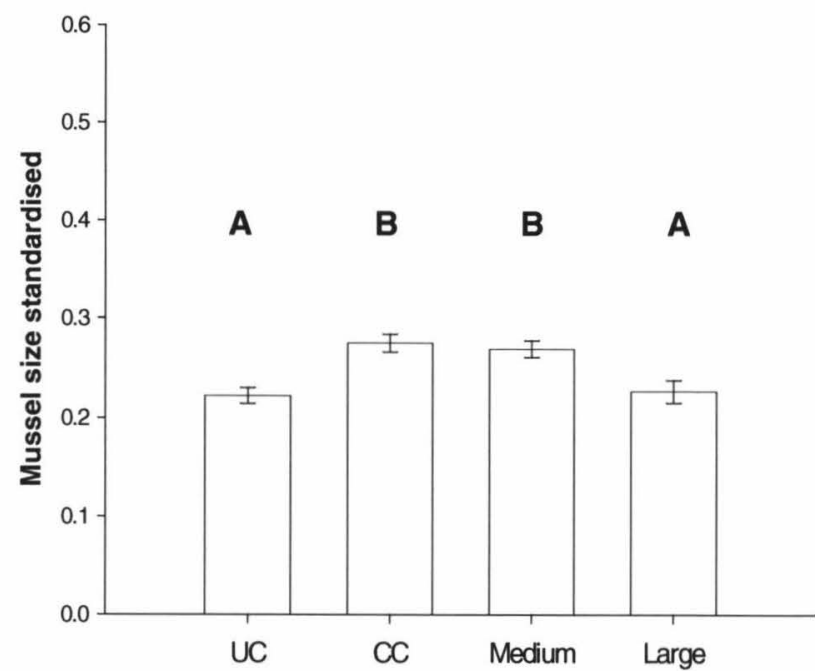

Laverique Bay

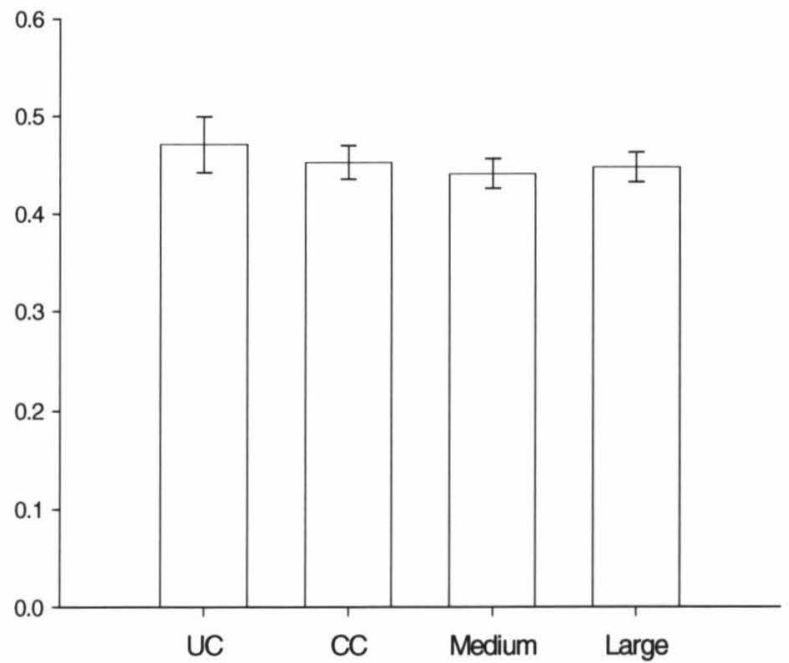

Fig 3.4: Effects of crab (N. minor) presence on P. canaliculus (GSM) size on spat catching ropes at the two study sites, Crail Bay and Laverique Bay. Mussel size was standardised for days by dividing the mussel number by the number of days the experiment ran for (42 and 77 days at Laverique Bay, 56 days for Crail Bay). The differences in P. canaliculus size were statistically significant at Crail Bay but not at Laverique Bay (ANOVA). Letters represent statistically significant post hoc groupings calculated using Tukey tests following ANOVA.

\subsubsection{Laboratory experiments}

\subsubsection{Size preference}

The null hypothesis that there was no significant difference in the percentage of mussels consumed in each mussel size class was rejected for male $(p<0.001)$ and female $(p$ $<0.001)$ crabs. However in the control treatments there were no significant differences in the number of GSM spat lost in relation to mussel size class (Table 3.5)

Table 3.4: Kruskal-Wallis non-parametric ANOVA result table: the grouping variable for this test was GSM size $(<5 \mathrm{~mm}, 5-10$ $\mathrm{mm}, 10-15 \mathrm{~mm},>15 \mathrm{~mm})$. Significant results are indicated in bold.

GSM SIZE PREFERENCE

\begin{tabular}{lccl} 
FACTOR & DF & $X^{2}$ & $P$-VALUE \\
\hline \hline FEMALE & 3 & 62.569 & $<0.001$ \\
MALE & 3 & 17.992 & $<0.001$ \\
CONTROL & 3 & 2.871 & 0.412
\end{tabular}


Female crabs consumed more mussels in the $<5 \mathrm{~mm}$ and $5-10 \mathrm{~mm}$ size classes than in the two larger mussel size classes $(10-15 \mathrm{~mm}$ and $>15 \mathrm{~mm})$, whereas the male crabs consumed more mussel spat in the 5-10 $\mathrm{mm}$ and $10-15 \mathrm{~mm}$ size classes (Fig 3.7). Female crabs showed a marked proportional decrease in the percent of mussel spat consumed from the $10-15 \mathrm{~mm}$ size class to the largest mussel size class $(-43 \%)$. The proportional decrease in mussel spat consumption between these two size classes was less substantial for male crabs (-29\%) (Fig 3.7).

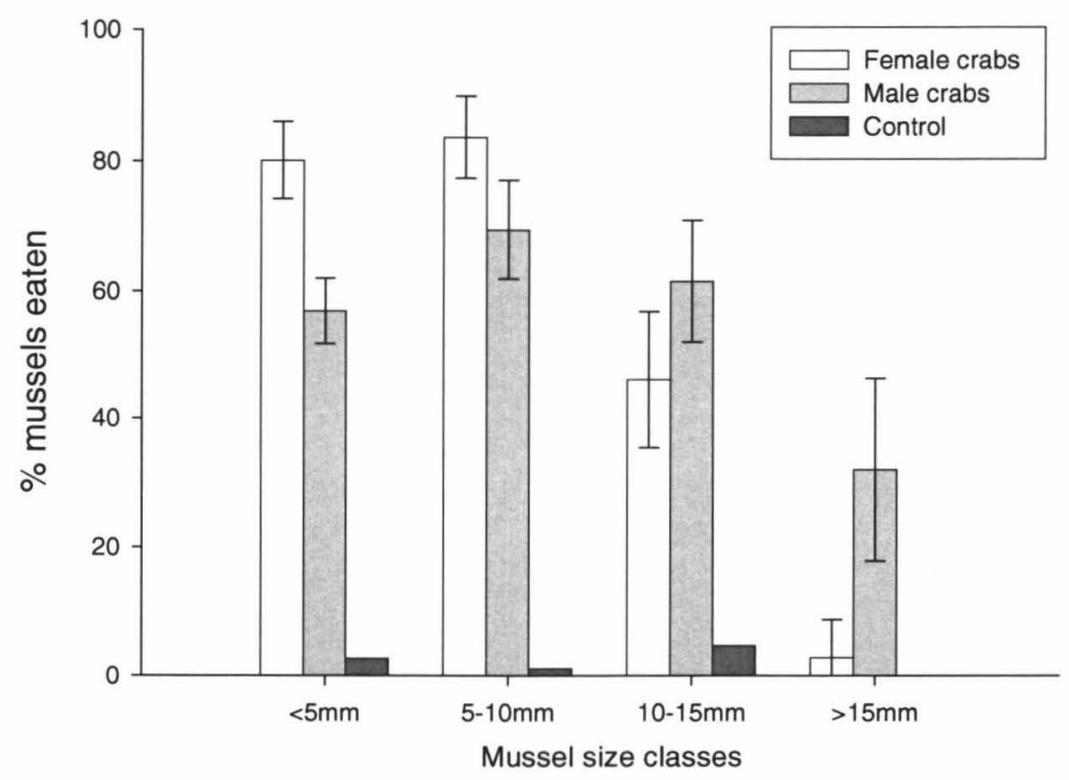

Fig 3.5: Average percent of GSM spat consumed by medium-sized male and female $N$. minor (males 20-35 mm TCW; females 15-27 mm TCW) and the number of mussels lost in control treatments (no crabs) over three days. Means $\pm 95 \%$ CI.

\subsubsection{Satiation trial}

The maximum mean number of GSMs eaten $\mathrm{crab}^{-1} \mathrm{hr}^{-1}$ was $56.43( \pm 13.02(95 \%$

C.I.)), at time one on the first day of experimentation. The mussel consumption rate by the crabs decreased over time. Per hour, this decrease was irregular (Fig 3.5), but the 
observed decrease in mussel consumption by the crabs from day 1 to day 3 was statistically significant (df: $2, F: 8.875, p$-value: $<0.001)$.

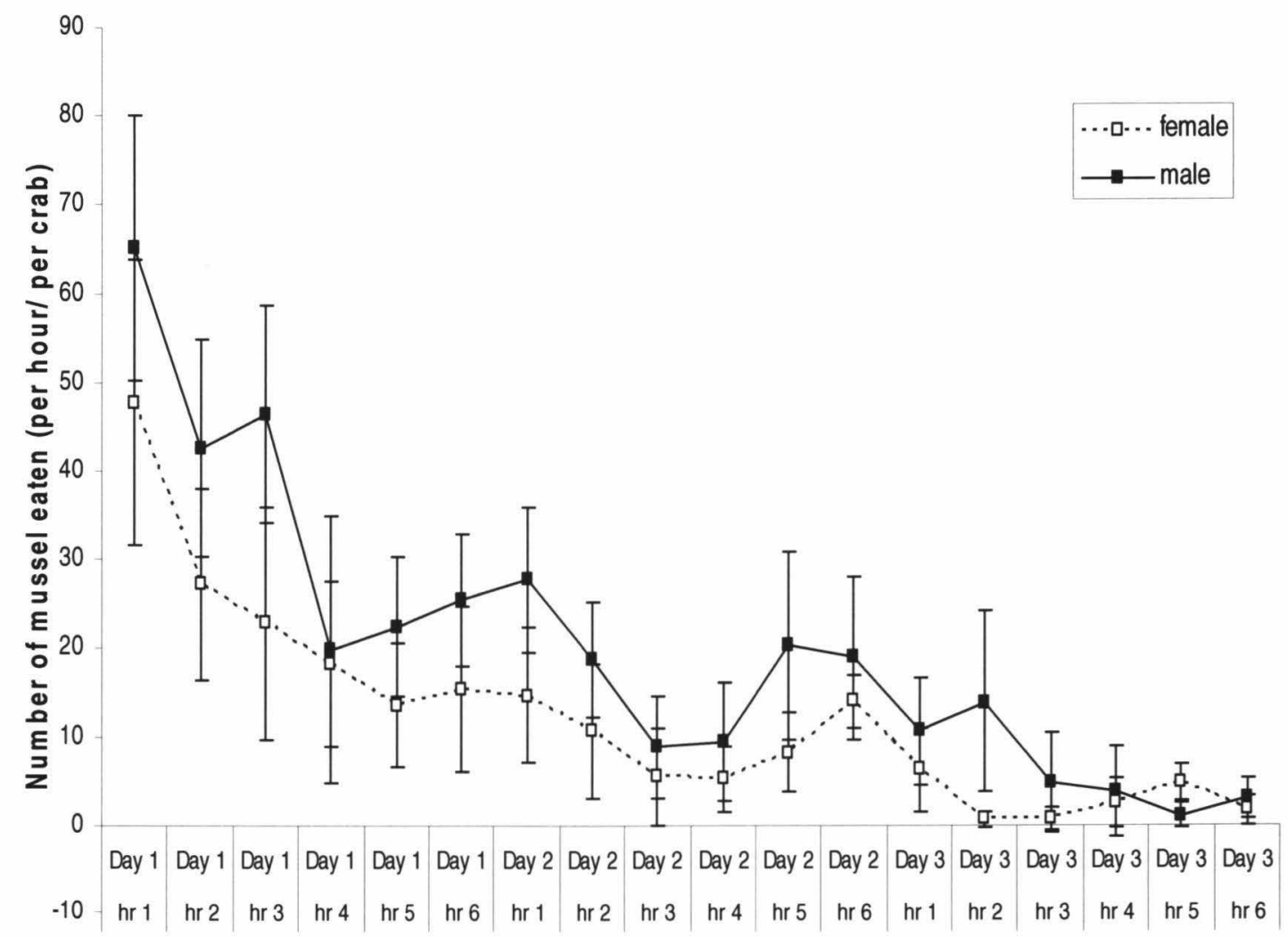

Fig 3.6: Average number of Greenshell ${ }^{\mathrm{TM}}$ mussel ( $P$. canaliculus) spat $(<5 \mathrm{~mm})$ eaten per medium sized male and female crab ( $N$. minor minor: males: $20-35 \mathrm{~mm}$ TCW, females: $15-27 \mathrm{~mm}$ TCW) per hour across three days of testing. Error bars are $95 \%$ confidence intervals of the mean.

Crab sex had a significant effect on the number of GSM spat consumed by medium sized N. minor (Fig 3.7, Table 3.5). Male crabs consumed significantly more GSM spat than female crabs, particularly on day one (Fig 3.7). However both male and female crabs consumed significantly less mussel spat with increasing time (Fig. 3.7). 


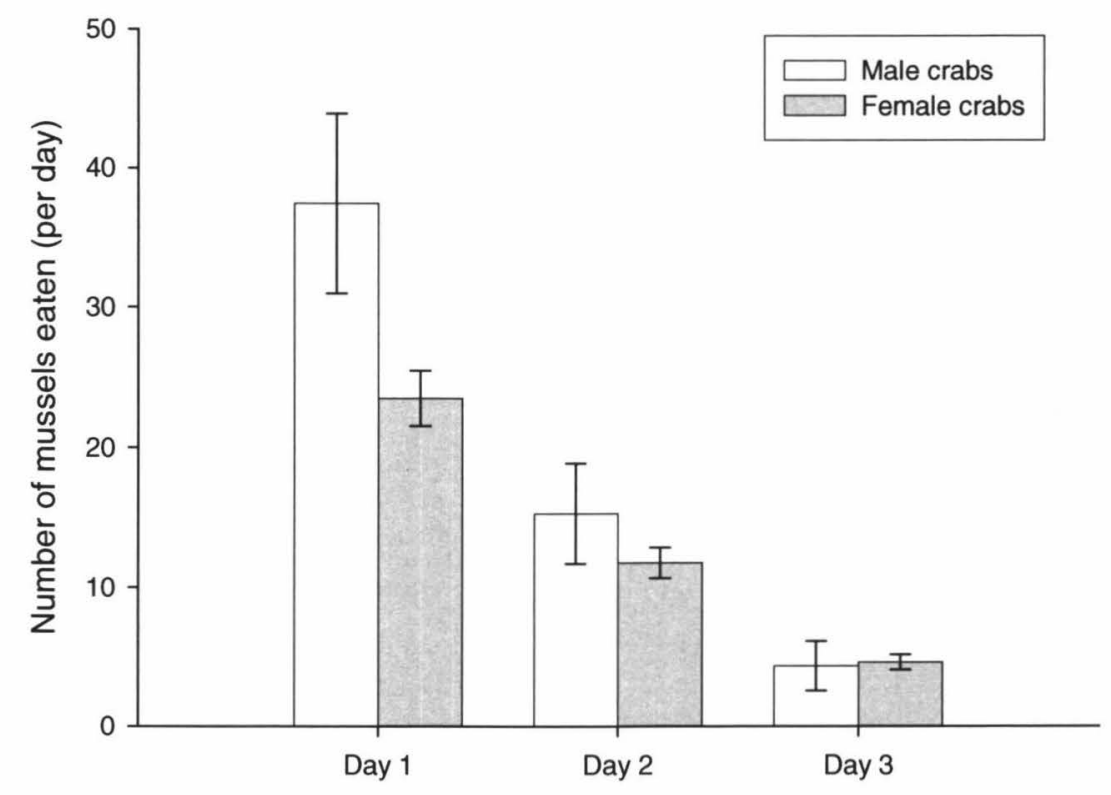

Fig 3.7: Average number of GSM ( $P$. canaliculus $)$ spat $(<5 \mathrm{~mm})$ eaten by male and female medium sized crabs ( $N$. minor: males: $20-35 \mathrm{~mm}$ TCW, females: $15-27 \mathrm{~mm}$ TCW) per day across three days of testing. Means $\pm 95 \%$ CI.

The proportional decrease in average mussel consumption was larger for male crabs (day 1-day 2: $59 \%$, day 2-day 3: $72 \%$ ) than for female crabs (day 1-day 2: $50 \%$ day 2-day 3: 61\%) and decreased more from day 2 to day 3 than from day 1 to day 2 (Fig 3.7). The interaction between day and crab sex was also significant (Table 3.5), so the difference between the number of mussel spat eaten by male and female crabs was more pronounced on the first day of the experiment as opposed to day three (Fig. 3.7, Table 3.5). 
Table 3.5: ANOVA table for proportion of GSM spat $(<5 \mathrm{~mm})$ eaten per day for medium sized male and female $N$. minor. The trial ran for three days and crabs were fed 100 mussel spat per crab per hour. Significant results indicated in bold. $F$-ratios calculated from Type III SS

\begin{tabular}{c|c|c|c|c}
\hline \multirow{2}{*}{ FACTOR } & \multicolumn{4}{|c}{ NUMBER OF MUSSEL SPAT EATEN } \\
\cline { 2 - 5 } & DF. & MS & $\boldsymbol{F}$ & P-VALUE \\
\hline CRAB SEX & 1 & 27.516 & 91.922 & $\mathbf{0 . 0 0 3}$ \\
DAY & 2 & 285.009 & 8.875 & $<0.001$ \\
CRAB SEXX DAY & 2 & 10.916 & 3.521 & $\mathbf{0 . 0 3 1}$ \\
\hline
\end{tabular}

\subsection{DISCUSSION}

GSM density on spat farm droppers in Pelorus Sound decreased significantly when decorator crabs $(N$. minor) were introduced. This decrease only occurred when small (mean $\pm 95 \%$ C.I. of $14.24 \mathrm{~mm} \pm 0.28$ ) Kaitaia spat were used in experiments during the winter of 2006 , than in the trials run on larger $(24.48 \pm 0.32)$ locally caught spat during the summer of $2006 / 2007$. GSM spat supply and retention from seeding to harvest is highly variable; losses are often more than $50 \%$ and losses of $95 \%$ are experienced regularly (Sim-Smith 2006, Carton et al. 2007). This inefficient use of GSM spat can lead to large production and financial losses (Carton et al., 2007). The mechanisms related to spat retention are complex and factors including nutrition (Buchanan 1994), water flow (Alfaro 2005), desiccation stress (Carton et al. 2007), disease (Jeffs et al. 1999), and settlement surface (Buchanan 1994, Alfaro and Jeffs 2003) have been implicated. This study adds the presence of a potential predator to the list of factors affecting spat retention, with the specific aim of helping to resolve the problem of spat losses. 
Predation by $N$. minor is not the only explanation for the observed decreases in GSM spat number in the presence of $N$. minor. The two study sites had similar physical characteristics, however the seasonality of the two trials could also be contributing to the observed differences in mussel spat losses. The number of storm activities and corresponding increases in hydrodynamic forces acting on the mussel spat increase during winter months. The risk of mussel spat dislodgement from the substrate is higher during stormy conditions, storms have been shown to cause losses of upto $65 \%$ of Mytilus califorianus (Paine and Levin 1981). The fact that in the current study the winter trial site, Crail Bay, had higher spat losses could have been due to the fact that the probability of dislodgement increases with increased hydrodynamic forces (Hunt and Scheibling 2001). Another explanation for the observed mussel spat losses is the ability of GSM spat to go through a secondary settlement phase if the primary settlement location becomes less than optimal (Buchanan 1994, Buchanan and Babcock 1997, Carton et al. 2007). During secondary settlement the spat will sever the byssus threads used to attach to a substrate during primary settlement and migrate over $100 \mathrm{~s}$ of meters by 'mucus- drifting' (Buchanan 1994). GSM spat can use secondary settlement to move a number of times before they recruit into grow-out areas (Jeffs et al. 1999). The ability for 'mucus-drifting' by GSM spat is lost once they reach $6 \mathrm{~mm}$ TSL, due to anatomical changes of the pedal glands (Buchanan and Babcock 1997). There is still a high level of uncertainty surrounding the factors that trigger secondary settlement in GSM spat, although stressors such as desiccation, temperature, starvation (Carton et al., 2007), and the presence of potential predators could be incentive enough for spat to invest effort into secondary settlement. 
Secondary settlement by GSM spat in the presence of $N$. minor could be classified as an anti-predator response, with the spat possibly responding to water-borne chemical cues emitted by the predatory crabs. The release of chemicals such as pheromones and amino acids has been documented for various species of crabs (Ryan, 1966), and these chemicals make it possible for prey species to detect predators (Côte, 1995). Predator avoidance behaviours have been linked to the chemical cues emitted by predators and chemicals secreted by conspecifics attacked by predators (Côte 1995, Reimer and Harms-Ringdahl 2001): secondary settlement might be an example of such an escape mechanism.

The decrease in both GSM spat numbers when $N$. minor were present could also be due to direct predation by the decorator crabs. Anecdotal evidence from mussel growers in the Marlborough Sounds and researchers at The Cawthron Institute (Nelson) suggests that $N$. minor found on mussel lines can consume substantial quantities of mussel spat. The current study supports these claims, with crabs in a laboratory setting consuming significant numbers of GSM spat (max. 36 GSM spat $\mathrm{h}^{-1} \mathrm{crab}^{-1}$ ). However, no alternative food-sources were offered to crabs so these results may over-estimate the predation rates of $N$. minor on GSM spat in a natural setting.

Woods (1993) researched the natural diet of $N$. ursus and found that it comprised of a wide range of items including: algae, gastropods, isopods, amphipods, bryozoans, sponges, bivalves and small decapods. Current evidence therefore suggests that bivalves typically only constitute a small portion of the diet of decorator crabs. This may be due to the morphology of the chelae which are generally elongate and slender (Wicksten 1980, Woods 1993) and are not well adapted to the crushing behaviour required to break the relatively thick shells of bivalves (Woods 1993) $N$. minor generally have to adopt 
one of two more labour intensive feeding techniques to prey on bivalves : 1) chipping or biting the shell margin using chelae or mandibles, or 2) forcing the valves of the bivalve apart using the chelae or legs (Woods 1993); pers. obs.).

Small mussels have thinner shells and could therefore be more attractive to $N$. minor, in the sense that thinner-shelled mussels are easier prey items than thicker-shelled mussels. This statement is supported by evidence from both the field and lab experiments in this study. At Crail Bay, where the mussels were smaller, the decrease in mussel density was greater than at Laverique Bay where the mussels were on average 10 mm larger (TSL post trial). In the laboratory trials, both male and female crabs consumed mussels across all of the size classes, however crabs preferred mussels $<15$ mm TSL and seemed to only consume larger individuals when the smaller mussels had become depleted.

Five major hypotheses exist in the literature which offers explanations for the observed size selection of GSM by decorator crabs. 1) the chelal wear model (Smallegange and van der Meer 2003), in which crabs select smaller than optimal mussels to prevent claw wear and tear.. 2) the prey evaluation hypothesis (Elner and Hughes 1978, Jubb et al. 1983), in which the crabs pick up and evaluate the prey item for a set period of time before accepting or rejecting it. When unlimited prey items are available the crabs select mussels close to the predicted optimal size, and as the optimal prey items become depleted the crabs will progressively take less profitable prey in accordance with the energy maximization model (Charnov 1976). 3) the 'relativestimulus' model (Jubb et al. 1983), in which the acceptance of the prey item is dependant on the strength of the olfactory and/or tactile stimuli emitted by a prey item when held in the chelae compared to the stimuli received from other prey items 
simultaneously touched by the crabs legs. If any breakage of the mussel shell occurs during handling the olfactory and tactile stimuli increase, making it highly likely that the crab will include this specific prey item in its diet. 4) the 'mechanical selection' model (Hughes and Seed 1981), in which crabs have difficulty grasping small mussels and often drop the prey item accidentally during manipulation. The extended period of time required to break into large mussels often exceeds the crabs' stimulus to persevere. Therefore crabs often passively select medium-sized prey items. 5) the 'key-stimulus' model (Hughes and Seed 1995), as in the 'relative-stimulus' model crabs select prey in response to the strength of the stimuli emitted by the prey item, rejection of the prey item will occur after a period of handling if the attack is proving ineffective. Previous experience by the predator will lead to adjustment of the handling time to suit the expectation of the quality and density of local prey populations (Hughes and Seed 1995).

The observed preference by male crabs $(N$. minor $)$ for GSM $<15 \mathrm{~mm}$ TSL and female crabs for GSM $<10 \mathrm{~mm}$ can be explained by all the aforementioned hypotheses, although some may be more relevant than others. For example, the 'chelal wear' hypothesis is particularly applicable in the current study. Mature $N$. minor males and females have sexually dimorphic chelae. As in many species of decorator crabs, both male and female chelae are long and slender (Wicksten 1980). However, chelae of male N. minor are generally larger and presumably stronger than those of females (pers. obs.), therefore male $N$. minor are better equipped to attack and consume a large size range of prey items (Woods 1993). The experimental crabs were starved before initiation of the size preference trials and the 'key stimulus' hypothesis allows for hunger levels in its design. Previous studies of decorator crab dietary preferences also support other aspects of this hypothesis. Woods (1993) found that dietary preferences of Notomithrax ursus 
were associated with crab age. Immature, possibly less experienced, $N$. ursus favoured small, easily handled prey such as isopods, amphipods and small gastropods, whereas mature crabs preferred large and more 'difficult' prey items such as calcareous red algae and decapods.

Many studies have investigated the predator-prey relationship between crabs and bivalves (Bertness and Cunningham 1981, Hughes and Seed 1981, Blundon and Kennedy 1982, Jubb et al. 1983, Lin 1991, Allen 1997, Barbeau et al. 1998, Leonard et al. 1999). However, very little published literature exists on the feeding behaviour and preferences of Notomithrax species which is surprising because they are common and widespread around New Zealand, and N. minor is particularly abundant on mussel farms in the Marlborough Sounds. Woods (1993) observed that bivalves only constitute a minor dietary component of the diet of the decorator crab N. ursus. However due to the methodology used to determine gut contents and the crabs' feeding behavior, the importance of bivalves as a dietary component was most likely underestimated. The current study encompasses both field and laboratory experimentation to comprehensively examine the relationship between the commercially important bivalve GSM and a potential predator $N$. minor. The results of this study indicate that contrary to previous studies on decorator crab dietary preferences, GSM's are likely a significant dietary component for $N$. minor found on GSM farms in the Pelorus Sound, Marlborough Sounds, New Zealand.

As in most organisms, crab prey selection and feeding rate is related to a number of motivational factors including activity level, age, moult stage, reproductive condition and hunger (Zimmer-Faust 1989, Hayden et al. 2007). In a study by (Brousseau et al. 2001) investigating the predation rates of the Asian crab (Hemigrapsus sanguineus) 
daily mean consumption rates of $12.7 \pm 11.6$ juvenile Mytilus edulis per day. This was similar to consumption rate of $13.7 \pm 2.9$ per day small ribbed mussels (Geukensia demissa) by large (CW 42-43mm) Panopeus herbstii (Brousseau et al. 2001). However in the current study the daily mean predation rate was significantly higher on day one of the trial (30.6 \pm 4.55 (95\% C.I.) GSM spat per day, sexes pooled; $N=15$ ) however this rate dropped off significantly over the three days of the trial to $4.5 \pm 1.47$ GSM spat per day. Although the current study indicates that $N$. minor is capable of consuming large numbers of GSM spat when starved in laboratory conditions, these results do not take into account the time spent foraging in the field that will possibly cause a significant reduction in the daily consumption rate. However due to the fact aquaculture practices lead to artificially high densities of juvenile GSMs laboratory consumption rates may provide a realistic estimate of the predatory impact of $N$. minor on GSM farms in the Marlborough Sounds.

When GSM spat are seeded onto farm lines the size range of the mussels will be limited to 4-6 mm TSL. It is however unknown when and how the crabs settle onto the farm lines. Prey size preferences of $N$. minor may not be important in an actual mussel farming situation if the crabs settle onto the mussel lines when the mussel spat have already grown to a size that is above the preferred prey size range. The growth rates of $N$. minor and GSM spat will also impact on the vulnerability of the spat to predation by the crabs, because size refuges may exist for mussel spat. The observed prey size preferences by $N$. minor will be the most problematic to mussel farmers when crabs within the experimental size range are found in high densities on spat farms where the GSM spat is within the crabs' preferred size range (i.e. $<15 \mathrm{~mm}$ TSL). Although this 
situation has been observed by individuals in the GSM industry it would be beneficial to know how often and where large $N$. minor occur on small GSM spat.

However due to fact that the experimental designs of the field and laboratory trials were constrained by time, spat availability and industry requirement, there are a number of opportunities for the GSM industry to expand on the current study. Firstly this study does not incorporate information regarding settlement patterns, growth rates and numbers of $N$. minor on mussel farms in the Pelorus Sound. These factors are important to determine whether medium sized crabs occur regularly on optimally sized mussels ( $>15 \mathrm{~mm}$ TSL), or if due to natural settlement and growth patterns, sporadic or constant size refuges exist for GSM spat. Secondly, the design of the field experiments does not allow the opportunity to distinguish between secondary settlement and predation as the underlying cause of the observed spat losses. Being able to determine these underlying causes may be interesting from an ecological point of view, but GSM growers are more interested in the fact large spat losses occur in the presence of $N$. minor rather than the causes of the losses. By supplementing the field trials in the current study with laboratory trials to determine the rate of secondary settlement such as those used by Meder et al. (2004), a more comprehensive understanding of the underlying causes of GSM spat losses in the presence of decorator crabs could be gained.

Despite the aforementioned areas of deficiency, this study demonstrates that decorator crab (N. minor) presence significantly decreases the retention rates of GSM spat on mussel farms in the Marlborough Sounds. The importance of this finding is amplified by the lack of published data regarding the focal predator and the economic significance of the GSM. Spat retention rates have been a contentious issue for GSM 
farmers since the birth of the industry and cost mussel farmers hundreds of thousands of dollars annually. Previous attempts to understand this issue have focused on a number of factors including predation (Sim-Smith 2006), competition (Hickman 1979, Hayden 1984) and environmental conditions (Alfaro 2005, Sim-Smith 2006). However this study highlights one factor affecting spat retention that has been previously overlooked and deserves closer scrutiny and experimental testing by the GSM industry in the Marlborough Sounds. 


\section{Chapter 4: General Conclusions}

\subsection{SUMMARY OF FINDINGS}

Quantitative field and laboratory studies of the effects of the decorator crab Notomithrax minor on GSM spat survival and attachment strength suggest that $N$. minor may cause significant financial losses to GSM farmers in the Marlborough Sounds, and elsewhere in NZ.

\subsubsection{N. minor and GSM spat byssus production}

Abiotic factors such as wave action, temperature and salinity have been identified as factors that affect the byssal characteristics and attachment strength of several mussel species (Young 1985, Côte 1995, Alfaro 2005). This study proposes that a biotic factor, specifically the presence of a potential predator ( $N$. minor), also affects byssus production and attachment strength in the spat of the commercially important mussel Perna canaliculus (GSM). The increase in byssal attachment strength and byssal thickness in the presence of $N$. minor was most pronounced in small, recently seeded GSM spat. Small mussels are most vulnerable to decorator crab predation (Chapter 3), and attachment strength is inversely related to prey profitability and attractiveness (Lin 1991).

Increased byssus production by GSM spat has a number of direct and indirect implications for GSM farmers. First, there are a number of published articles that suggest byssus production can account for a significant proportion of carbon, nitrogen and energy allocated to growth (Griffiths and King 1979, Hawkins and Bayne 1985). This study was not specifically designed to assess the energetic costs of byssus production in $P$. canaliculus but it is realistic to assume some trade-off with growth will 
exist to produce more and thicker byssus threads. Reduced growth rates will increase costs to GSM farmers due to decreased crop turnover and the consequent inefficient use of space and resources. Second, the retention rates of GSM spat on farms in the Marlborough Sounds are regularly less than 5\%. GSM spat costs mussel farmers between NZ\$0.80 (Kaitaia spat) and NZ\$1.00 (locally caught and Golden Bay spat) per $\mathrm{m}$ of spat rope (at a density of $200 \mathrm{GSMs} \mathrm{m}^{-1}$ ) (pers. comm., Aaron Pannell, Marlborough Mussels Ltd., 2007). When high densities of $N$. minor were present on Kaitaia spat at Crail Bay the spat losses were at a maximum (Fig 3.3). At the levels observed in the field trials GSM spat losses translated to an approximate cost of NZ $\$ 30,000-\$ 35,000$ per average sized GSM farm (based on a seeding density of 200 GSM spat $\mathrm{m}^{-1}$, on average sized farm i.e. 90 backbones with $4000 \mathrm{~m}$ of line per backbone). The costs are amplified by farmers having to purchase excess GSM spat or spend more time and effort catching spat to compensate for the losses (Sim-Smith 2006). Third, spat attachment strength also has implications for spat survival, particularly during the stripping of spat for reseeding, because if the mussel spat are attached more tightly they are more likely to be crushed in the machinery used to strip spat off the droppers.

\subsubsection{N. minor predation and GSM spat retention}

$N$. minor presence not only affected the byssal characteristics of GSM spat but also spat retention rates, but not mussel size. The density of Kaitaia GSM spat on mussel farms in Pelorus Sound decreased significantly when medium and high densities of the decorator crabs, $N$. minor, were introduced. The mechanisms related to spat retention are complex, with factors implicated in substantial mussel spat losses including nutrition 
(Buchanan 1994), water flow (Alfaro 2005), desiccation stress (Carton et al. 2007), disease (Jeffs et al. 1999), and settlement surface (Buchanan 1994, Alfaro and Jeffs 2003). This study adds the presence of a predatory crab to the list of factors affecting spat retention and could therefore help resolve the issue of spat losses. GSM spat losses in the presence of $N$. minor may be due to detachment by GSM spat (i.e. secondary settlement) in response to chemical cues secreted by $N$. minor and/or direct predation pressure by the decorator crabs. Crab sex and size also affected the number of GSM spat consumed and prey size preference of $N$. minor; female crabs preferentially consumed smaller mussels than male crabs, however all of the crabs preferred mussels under 15 mm TSL and seemed to only consume larger individuals when the smaller mussels had become depleted. These results relate directly to GSM spat losses in the field because spat are seeded artificially onto spat grow-out droppers when they are between $40-50$ $\mathrm{mm}$ TSL. This is within the size range of GSM that $N$. minor prefers to consume and the size range during which secondary settlement of spat occurs (Buchanan and Babcock 1997). The current study does not distinguish between secondary settlement and predation as the underlying cause of the observed spat losses. Distinguishing between the two causes could be important from an ecological point of view, however GSM farmers are more interested in the fact that the losses occur and not the underlying mechanisms. Spat losses can cost commercial GSM farmers hundreds of thousands of dollars annually, due to farmers having to purchase more spat or spend more time and effort catching spat to compensate for the losses (Sim-Smith 2006). 


\subsubsection{General conclusions}

The goal of this study was to obtain new quantitative information regarding the effects of a common fouling species, the decorator crab $N$. minor, on farmed GSM spat in the Marlborough Sounds, NZ. N. minor were identified by growers in the GSM industry as a possible economic threat to mussel farmers. The results of the current study support this claim by demonstrating that small GSM spat can be highly susceptible to predation by $N$. minor, and that when $N$. minor are present on GSM spat farms, spat retention rates could drop significantly. The fact $N$. minor presence also increased the attachment rates of GSM spat that were not lost due to predation or secondary settlement also leads to increased costs to GSM farmers due to increased spat damage and loss during stripping and reseeding. Identifying $N$. minor as a major cause of reduced spat retention rates and the resulting financial losses is not realistic until the reproductive behaviour, growth, and distribution of $N$. minor is investigated further. Currently there is no single abiotic or biotic factor that completely explains the low GSM spat retention rates observed in the Marlborough Sounds. It is more likely that a complex array of factors influence retention rates and this study suggests that $N$. minor is a previously overlooked and underestimated factor that contributes significantly to spat retention issues faced by GSM farmers.

From an ecological perspective the results of the current study appear to contradict each other to some degree; $N$. minor increases both GSM attachment strengths and GSM spat losses. The majority of the spat in the field trials were lost due to predation or secondary settlement and the increase in byssal attachment strength reported in chapter 2 only applied to the GSM spat that were still present after experimentation. 
Due to the fact this study is highly industry focused it is important to note that although some ecological contradiction does occur, the evidence does indicate that there are two detrimental processes acting on GSM spat in the presence of $N$. minor that may have serious financial consequences for mussel farmers. Firstly, at an early GSM life stage, the presence of decorator crabs results in substantial GSM spat losses, either directly via predation or indirectly via spat detachment. Secondly, the surviving mussels have higher strength of attachment which means energy diverted from growth (i.e.production) into byssal attachment and that the mussels are more susceptible to damage during reseeding and harvesting processes.

\subsection{FUTURE DIRECTIONS}

The experimental designs of the field and laboratory trials were constrained by time, spat availability and industry requirement, which means that there are a number of opportunities to expand on the current study. The two field trials were conducted on different types of spat at different times of year; at Crail Bay (summer 2006/2007), small, recently seeded Kaitaia spat were used, and at Laverique Bay (winter 2006) the spat were larger, locally caught spat. This leads to problems when comparing predation rates between the sites particularly because the Kaitaia spat were well within the preferred size range of $N$. minor and the locally caught spat at Laverique Bay were already at the upper limit of size preference at the start of the field trial. Multiple trials at different sites run simultaneously on the same type and size of GSM spat could be used to build a more comprehensive picture of the potential implications of $N$. minor presence on GSM spat retention and byssal characteristics. 
Due to the design of the field experiments, the current study cannot distinguish between secondary settlement and predation as the underlying cause of the observed spat losses. By supplementing the field trials in the current study with laboratory trials to determine the rate of secondary settlement such as those used by Meder et al. (2004), a more comprehensive understanding of the underlying causes of GSM spat losses in the presence of decorator crabs could be gained. Being able to determine the underlying causes of spat losses may be interesting from an ecological point of view, but GSM growers are less interested in the causes, than in the fact that large spat losses occur in the presence of $N$. minor.

Very little published data are available regarding the diet, behaviour and growth of $N$. minor, which is surprising as it is common on GSM farming structures and has been identified by members of the mussel farming industry as a potential threat. Identifying the growth rates and settlement behaviour of $N$. minor on GSM farms in the Marlborough Sounds was beyond the scope of this study; however understanding the settlement behaviour of $N$. minor could lead to the development of measures that minimise the impact of this predator.

Fish predation of GSMs is minimised by holding small mussels near the water's surface, moving spat from areas of high to low fish density within the first 8 wk and reducing the amount of fouling and disturbance (Jeffs et al. 1999, Sim-Smith 2006). Similar precautions could be taken to minimise the settlement of $N$. minor onto GSM spat farms if the settlement and growth behaviour of this crab species is better understood. GSM farmers have experimented with caging of spat susceptible to predation with varying levels of success. Early trials included putting a plastic cage around each rope and enclosing entire farms in nets, the nets had a tendency to get 
blocked by fouling species and the mussel spat in the plastic cages migrated to the outside of the cage (Dawber 2004). Oyster and clam farmers in the Northern hemisphere also use plastic mesh and netting to cover bivalve beds and exclude crustacean predators. The predator netting excludes adult crabs but small juveniles often still enter the cages and grow rapidly to a size where they are able to consume significant numbers of older bivalves (Flimlin 1993). Caging of GSM spat lines for short periods of time soon after seeding or reseeding when the mussels are most vulnerable to predation could significantly reduce the numbers of $N$. minor present. However due to the cryptic nature of the decorator crabs it would be difficult to detect any juveniles that had made their way into the cages. Regular visual inspection of spat lines for the occurrence of high densities of larger $N$. minor and the subsequent manual removal of the crabs is another possible risk management procedure. Many bivalve culturists have employed traps to capture and lure away predatory crabs from mussel lines and bivalve culture plots, a good understanding of the dietary preferences of $N$. minor is essential when using crab traps. Using traps has the potential to attract more non-target predators to the area if the bait and trapping method is too generalised. A more radical method to eliminate or minimise $N$. minor numbers could include the use of chemicals. Acetic acid and chlorine dips of spat during reseeding have been used to control fouling species (Forrest et al. 2007), including the tunicate Styela clava (LeBlanc et al. 2007) and the sea-squirt Didemnum vexillum (Coutts and Sinner 2004). If $N$. minor settle onto the GSM spat lines before the final re-seeding, then similar treatments could also be used to eradicate the crabs. Research would be needed to determine the appropriate chemicals and concentrations required to remove or kill the crabs whilst causing the lowest acceptable damage to the GSMs. The development of hatchery techniques for artificially 
raising commercial quantities of mussel spat also offers the possibility to selectively breed families of GSM that are more resistant to crab predation.

N. minor is an omnivore and Woods (1993) suggested that bivalves may only constitute a small proportion of the diet of a related Notomithrax spp. The dietary preferences of $N$. minor should also be examined in a larger-scale experiment, because a number of other taxa, including fouling algae and bryozoan species, are present on GSM farms which may be preferred over GSM spat. Personal observations of the experimental sections of GSM spat droppers indicated that the density of fouling organisms in the high and medium crab density treatments was a significantly lower than in the caged and uncaged control treatments. $N$. minor might prefer to consume problematic fouling species such as the blue mussel Mytilus galloprovincialis over GSM making it a beneficial species to the GSM industry.

The results of this study provide novel and practical information describing the interaction between the decorator crab $N$. minor and GSM spat. It also assesses the threat this fouling species poses to an economically significant farmed bivalve. The findings of this study, and the recommendations arising from it, will be shared with the GSM industry to raise awareness of the previously under-estimated threat that crabs pose to GSM farms. 


\section{References}

Adler, F.R., Harvell, C.D., 1990. Inducible Defenses, Phenotypic Variability and Biotic Environments. Trends in Ecology and Evolution 5, 407-410.

Alfaro, A. C. 2005. Byssal attachment of juvenile mussels, Perna canaliculus, affected by water motion and air bubbles. Aquaculture 255:357-361.

Alfaro, A. C., and A. G. Jeffs. 2003. Variability in mussel settlement on suspended ropes placed at Ahipara Bay, Northland, New Zealand. Aquaculture 216:115-126.

Allen, D. G. 1997. Impact of invertebrate predation on the New Zealand Scallop (Pecten novaezelandiae). Victoria University of Wellington, Wellington.

Allen, J. A., M. Cook, D. J. Jackson, S. Preston, and E. N. Worth. 1976. Observations on the rate of production and the mechanical properties of the byssus threads of Mytilus edulis L. Journal of Molluscan Studies 42:279-289.

Apte, S., B. Star, and J. P. A. Gardner. 2003. A comparison of genetic diversity between cultured and wild populations, and a test for genetic introgression in the New Zealand mussel Perna canaliculus (Gmelin 1791). Aquaculture 219:193-220.

Baldwin, I. T. 1988. The alkaloidal responses of wild tobacco to real and simulated herbivory. Oecologia 77:378-381.

Barbeau, M. A., R. E. Scheibling, and B. G. Hatcher. 1998. Behavioural responses of predatory crabs and sea stars to varying density of juvenile sea scallops. Aquaculture 169:87-98.

Bell, E. C., and J. M. Gosline. 1996. Mechanical design of mussel byssus: material yield enhances attachment strength. The Journal of Experimental Biology 199:10051017.

Bennett, E. W. 1964. The Marine Fauna of New Zealand: Crustacea Brachyura. New Zealand Department of Scientific and Industrial Research Bulletin 153:119.

Bertness, M. D., and C. Cunningham. 1981. Crab shell-crushing predation and gastropod architectural defense. Journal of Experimental Marine Biology and Ecology 50:213-230.

Blundon, J. A., and V. S. Kennedy. 1982. Mechanical and Behavioural Aspects of Blue Crab, Callinectes sapidus (Rathbun), Predation on Chesapeake Bay Bivalves. Journal of Experimental Marine Biology and Ecology 65:47-65.

Brousseau, D. J., A. Filipowicz, and J. A. Baglivo. 2001. Laboratory investigations of the effects of predator sex and size on prey selection by the Asian crab, Hemigrapsus sanguineus. Journal of Experimental Marine Biology and Ecology 262:199-210.

Buchanan, S. J. 1994. The spat trap. Removing the risk from spat production. Pages 5455 Seafood N.Z.

Buchanan, S. J., and R. Babcock. 1997. Primary and secondary settlement by the Greenshell mussel Perna canaliculus. Journal of Shellfish Research. 16:71-76.

Carrington, E. 2002. Seasonal Variation in the Attachment Strength of Blue Mussels: Causes and Consequences. Limnology and Oceanography 47:1723-1733.

Carton, A. G., A. G. Jeffs, G. Foote, H. Palmer, and J. Bilton. 2007. Evaluation of methods for assessing the retention of seed mussels (Perna canaliculus) prior to seeding for grow-out. Aquaculture 262:521-527. 
Charnov, E. L. 1976. Optimal foraging: attack strategy of a mantid. American Naturalist 110:141-151.

Cheung, S. G., S. Lam, Q. F. Gao, K. K. Mak, and P. K. S. Shin. 2004. Induced antipredator responses of the green mussel, Perna viridis (L.), on exposure to the predatory gastropod, Thais clavigera Kuster, and the swimming crab, Thalamita danae Stimpson. Marine Biology 144:675-684.

Cheung, S. G. T., P.Y. Yip, K.M. Shin, P.K.S. 2004. Chemical cues from predators and damaged conspecifics affect byssus production in the green-lipped mussel Perna viridis. Marine and Freshwater Behaviour and Physiology 37:127-135.

Christophersen, G., and $\varnothing$. Strand. 2003. Effect of reduced salinity on the great scallop (Pecten maximus) spat at two rearing temperatures. Aquaculture 215:79-92.

Clarkson, R., P. Lupi, K. G. Heasman, and A. Coutts. 2003. New Zealand Mussel Industry Marine Fouling Organisms Guide. Page 33. New Zealand Mussel Industry Council Ltd.

Côte, I. M. 1995. Effects of predatory crab effluent on byssus production in mussels. Journal of Experimental Marine Biology and Ecology 188:233-241.

Côte, I. M., and E. Jelnikar. 1999. Predator-induced clumping behaviour in mussels (Mytilus edulis Linnaeus). Journal of Experimental Marine Biology and Ecology 235:201-211.

Coutts, A., and J. Sinner. 2004. An updated benefit-cost analysis of management options for Didemnum vexillum in Queen Charlotte Sound. 925, Cawthron Institute, Nelson.

Cruz-Rivera, E. 2001. Generality and specificity in the feeding and decoration preferences of three Mediterranean crabs. Journal of Experimental Marine Biology and Ecology 266:17-31.

Dawber, C. 2004. Lines in the water: a history of Greenshell mussel farming in New Zealand. October Enterprises Ltd., Picton, New Zealand.

Dolmer, P. 1998. The interactions between bed structure of Mytilus edulis L. and the predator Asterias rubens L. Journal of Experimental Marine Biology and Ecology 228:137-150.

Elner, R. W., and R. N. Hughes. 1978. Energy Maximization in the Diet of the Shore Crab, Carcinus maenas. Journal of Animal Ecology 47:103-116.

Flaws, D. E. 1975. Aspects of the biology of mussels in the Cook Strait area. Ph.D thesis. Victoria University of Wellington., Wellington, New Zealand.

Flimlin, G. 1993. Major Predators of Cultured Shellfish. Northeastern Regional Aquaculture Center 180.

Gibbs, M. M., S. E. Pickmere, P. H. Woods, G. W. Payne, and M. R. James. 1992. Nutrient and chlorophyll $a$ variability at six stations associated with mussel farming in Pelorus Sound, 1984-85. New Zealand Journal of Marine and Freshwater Research 26:197-211.

Griffin, D. J. G. 1966. The marine fauna of New Zealand: spider crabs, family Majidae (Crustacea, Brachyura). New Zealand Oceanographic Institute memoir 35.

Griffiths, C. L., and J. A. King. 1979. Energy expended on growth and gonad output in the ribbed mussel Aulacomya ater. Marine Biology 53:217-222.

Harvell, C. D. 1984. Predator-Induced Defense in a Marine Bryozoan. Science 224:1357-1359. 
Harvell, C. D. 1990. The Ecology and Evolution of Inducible Defenses. The Quaterly Review of Biology 65:323-340.

Hawkins, A. J. S., and B. L. Bayne. 1985. Seasonal variation in the relative utilization of carbon and nitrogen by the mussel Mytilus edulis: budgets, conversion efficiencies and maintenance requirements. Marine Ecology Progress Series 25:181-188.

Hayden, B. J. 1984. Mussel spat mortality. Catch 11:19-20.

Hayden, B. J. 1994. What do we know about greenshell mussel spat? part 1. Pages 45-47 Seafood N.Z.

Hayden, D., A. Jennings, C. Muller, D. Pascoe, R. Bublitz, H. Webb, T. Breithaupt, L. Watkins, and J. Hardege. 2007. Sex-specific mediation of foraging in the shore crab, Carcinus maenas. Hormones and Behaviour 52:162-168.

Hickman, R. W. 1979. Allometry and Growth of the Green-Lipped Mussel Perna canaliculus in New Zealand. Marine Biology 51:311-327.

Hickman, R. W., J. H. Waite, J. Illingworth, J. L. Meredith-Young, and G. Payne. 1991a. The relationship between farmed mussels, Perna canaliculus, and available food in Pelorus-Kenepuru Sound, New Zealand. Aquaculture 99:49-68.

Hickman, R. W., R. P. Waite, J. Illingworth, J. L. Meredith-Young, and G. Payne. 1991b. The ralationship between farmed mussels, Perna canaliculus, and available food in Pelorus-Kenepuru Sound, New Zealand, 1983-1985. Aquaculture 99:49-68.

Hughes, R. N. 1979. Optimal Diets under the Energy Maximization Premise: The Effects of Recognition Time and Learning. The American Naturalist 113:209221.

Hughes, R. N., and R. Seed. 1981. Size selection of mussels by the blue crab Callinectes sapidus, energy maximizer or time minimizer? Marine Ecology Progress Series 6:83-89.

Hughes, R. N., and R. Seed. 1995. Behavioural mechanisms of prey selection in crabs. Journal of Experimental Marine Biology and Ecology 193:225-238.

Hunt, H. L., and R. E. Scheibling. 2001. Predicting wave dislodgment of mussels: variation in attachment strength with body size, habitat, and season. Marine Ecology Progress Series 213:157-164.

Jeffs, A. G., R. C. Holland, S. H. Hooker, and B. J. Hayden. 1999. Overview and bibliography of research on the greenshell mussel, Perna canaliculus, from New Zealand waters. Journal of Shellfish Research. 18:347-360.

Jenkins, R. J. 1979. Mussel Cultivation in the Marlborough Sounds (New Zealand). Page 75pp. in D. F. J. Ltd., editor. 1979. David F. Jones Ltd., Wellington, New Zealand.

Jubb, C. A., R. N. Hughes, and T. Rheinallt. 1983. Behavioural mechanisms of sizeselection by crabs, Carcinus maenas (L.) feeding on mussels, Mytilus edulis L.

. Journal of Experimental Marine Biology and Ecology 66:81-87.

Kilar, J. A., and R. M. Lou. 1986. The subtelties of camouflage and dietry preference of the decorator crab, Microphrys bicornutus Latrielle (Decapoda: Brachyura)* Journal of Experimental Marine Biology and Ecology 101:143-160.

LeBlanc, N., J. Davidson, R. Tremblay, M. McNiven, and T. Landry. 2007. The effect of anti-fouling treatments for the clubbed tunicate on the blue mussel, Mytilus edulis. Aquaculture 264:205-213. 
Leonard, G. H., M. D. Bertness, and P. O. Yund. 1999. Crab Predation, Waterborne Cues, and Inducible Defenses in the Blue Mussel, Mytilus edulis. Ecology 80:114.

Lin, J. 1991. Predator-prey interactions between blue crabs and ribbed mussels living in clumps. Estuarine, coastal and shelf science. 32:61-69.

Mistri, M. 2004. Predatory behaviour and preference of a successful invader, the mud crab Dyspanopeus sayi (Panopeidae), on its bivalve prey. Journal of Experimental Marine Biology and Ecology 312:385-398.

Paine, R. T. 1969. The Pistaster-Tegula Interaction: Prey Patches, Predator Food Preference and Intertidal Community Structure. Ecology 50:950-961.

Paine, R. T., and S. A. Levin. 1981. Intertidal landscapes: disturbance and the dynamics of pattern. Ecological Monographs 51:145-178.

Powell, A. W. B. 1979. New Zealand Mollusca: Marine, Land and Freshwater Shells. Collins, Auckland, New Zealand.

Reimer, O., and S. Harms-Ringdahl. 2001. Predator-inducible changes in blue mussels from the predator-free Baltic Sea. Marine Biology 139:959-965.

Reimer, O., and M. Tedengren. 1996. Phenotypical Improvement of Morphological Defences in the Mussel Mytilus edulis Induced by Exposure to the Predator Asterias rubens. Oikos 75:383-390.

Sato, M., and K. Wada. 2000. Resource utilization for decorating in three intertidal majid crabs (Brachyura: Majidae). Marine Biology 137:705-714.

SeaFIC. 2007. Exports of Seafood Produce for 12 Months ending December 2006.in E. o. S. P. f.-p. http://www.seafood.co.nz/f403, editor., NZ Seafood Industry Council.

Siddall, S. E. 1980. A clarification of the genus Perna (Mytilidae). Bulletin of Marine Science 30:858-870.

Sim-Smith, C. 2006. Greenshell ${ }^{\mathrm{TM}}$ mussels: solving the case of the disappearing spat. Water \& Atmosphere 14:16-17.

Smallegange, I. M., and J. van der Meer. 2003. Why do shore crabs not prefer the most profitable mussels? Journal of Animal Ecology 72:599-607.

Wear, R. G., and W. R. Webber. 1981. Life history studies on New Zealand Brachyura *. Larvae of the family Majidae. New Zealand Journal of Marine and Freshwater Research 15:331-383.

Webb, S. C., and K. G. Heasman. 2006. Evaluation of fast green uptake as a simple fitness test for spat of Perna canaliculus (Gmelin, 1791). Aquaculture 252:305316.

Wicksten, M. K. 1980. Decorator crabs. Scientific American 242:146-154.

Woods, C. M. C. 1993. Natural diet of the crab Notomithrax ursus (Brachyura: Majidae) at Oaro, South Island, New Zealand. New Zealand Journal of Marine and Freshwater Research 27:309-315.

Woods, C. M. C., and C. L. McLay. 1994. Use of camouflage materials as a food store by the spider crab Notomithrax ursus (Brachyura: Majidae). New Zealand Journal of Marine and Freshwater Research 28:97-104.

Young, G. A. 1985. Byssus-thread formation by the mussel Mytilus edulis: effects of environmental factors. Marine Ecology Progress Series 24:261-271.

Zimmer-Faust, R. K. 1989. The relationship between chemoreception and foraging behaviour in crustaceans. Limnology and Oceanography 34:1367-1374. 
References 